Copyright

by

Andrew Reed McNeese

2010 
The Thesis committee for Andrew Reed McNeese

Certifies that this is the approved version of the following thesis:

\title{
An Investigation of the Combustive Sound Source
}

\author{
APPROVED BY \\ SUPERVISING COMMITTEE:
}

Co-Supervisors:

Preston S. Wilson

David P. Knobles 


\title{
An Investigation of the Combustive Sound Source
}

Andrew Reed McNeese, B.S.M.E.

Thesis

\author{
Presented to the Faculty of the Graduate School \\ of the University of Texas at Austin \\ in Partial Fulfillment \\ of the Requirements \\ for the Degree of
}

Master of Science in Engineering

The University of Texas at Austin

August 2010 


\section{Acknowledgments}

I would like to thank Dr. Preston S. Wilson for his guidance and assistance throughout the work on this thesis. He was deserving of thanks for his help to me even before I began working on this project. His involvement and advice in my studies as a undergraduate and graduate student have helped make my time spent at UT a worthwhile experience. I would also like to thank Dr. David P. Knobles for his help and advice in conducting research and writing this thesis. I

will not forget the time I was able to spend with the two of them while testing the CSS.

Also, deserving much thanks are Ricky Lenhart, Rob Halter, Ira Morgan, and the entire ESL E-team for their continual help throughout the development of this source. Their patience in working with me, and their development of the hardware, electronics, and preliminary testing are the reason this project has been able to continue to progress. I am indebted to each of these individuals for the help and skills they have given me.

Jason Sagers is also due much appreciation, not only for his assistance on this project but for his time spent as a fellow student, office mate, and friend. His willingness to not only help me with this project, but with homework and anything I could ask for is greatly appreciated. His hard work, knowledge, and values have made me a better engineer.

I would also like to thank my parents, Kyle and Judy, as well as my sister, Kelsey, for all the support throughout the years. Without their willingness to sacrifice for my sake, none of this would have been possible. I cannot give them enough thanks.

Financial support for this project was provided by the Applied Research Laboratories Internal Research and Development program, and by Space and Naval Warfare Systems Command PEO C4I, PMW 120.

June, 2010 


\title{
An Investigation of the Combustive Sound Source
}

\author{
by \\ Andrew Reed McNeese, MSE \\ The University of Texas at Austin, 2010 \\ CO-SUPERVISOR: Preston S. Wilson \\ CO-SUPERVISOR: David P. Knobles
}

This thesis describes the development and testing of the Combustive Sound Source (CSS), which is a broadband underwater sound source. The CSS is being developed as a clean, safe, and cost effective replacement to underwater explosive charges, which exhibit an inherent danger to marine life and researchers using the charges. The basic operation of the CSS is as follows. A combustible mixture of gas is held below the surface of the water in a combustion chamber and ignited with an electric spark. A combustion wave propagates through the mixture and converts the fuel and oxidizer into a bubble of combustion products, which expands due to an increase in temperature, and then ultimately collapses to a smaller volume than before ignition, producing a high intensity, low frequency acoustic signal. The thesis begins by discussing the background, history, and purpose of developing the CSS. It continues by describing the current apparatus and the essential components and convenient features added to the latest mechanical design. The general operation is discussed along with a description of an experiment conducted to determine the acoustic output and robustness of the current CSS. The results of this experiment are presented in terms of the effect of volume, ignition depth, oxidizing gas, combustion chamber size, and repeatability of acoustic signatures. Discussion of apparatus robustness is presented to suggest improvements for future CSS designs. 


\section{Contents}

Chapter 1 Introduction 1

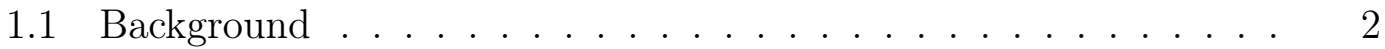

1.2 Literature Review . . . . . . . . . . . . . . . . . . . . . 3

1.2.1 The Combustion Process . . . . . . . . . . . . . 3

1.2.2 Parametric Analysis of Gas Volume, Ignition Depth, and Gas Mixture .................. 5

1.2.3 Versatility . . . . . . . . . . . . . . 6

1.3 Purpose of Work . . . . . . . . . . . . . . . . . . . . 7

1.4 Source Level Metrics . . . . . . . . . . . . . . . . . . . . 8

1.5 Rayleigh-Willis Bubble Model . . . . . . . . . . . . . . 12

1.6 Thesis Organization . . . . . . . . . . . . . . . . . . . . 15

$\begin{array}{lll}\text { Chapter } 2 & \text { Description of Apparatus } & 17\end{array}$

2.1 Combustion Chamber . . . . . . . . . . . . . . . . . 17

2.2 Gas Delivery System . . . . . . . . . . . . . . . . . 19

2.3 Ignition System . . . . . . . . . . . . . . . . . . . . 23

2.4 Additional System Components . . . . . . . . . . . . . . 26

2.4 Power ...................... . . 26

2.4 Vent ......................... . . 27

2.4.3 Water Level Sensor . . . . . . . . . . . . . . . . 28

2.4.4 Recording System and Electronic Controls . . . . . . . . . 29

Chapter 3 Description of Experiments 30

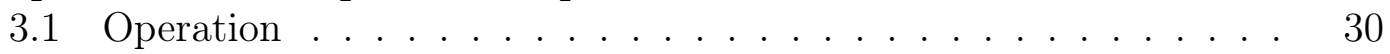

3.2 Description of Experiment . . . . . . . . . . . . . 33

$\begin{array}{lll}\text { Chapter } 4 & \text { Results } & \mathbf{3 6}\end{array}$

4.1 Effect of Increasing Volume . . . . . . . . . . . . . . . . . 36

4.2 Effect of Increasing Depth . . . . . . . . . . . . . . . . . . 37

4.3 Effect of Oxidizing Gas . . . . . . . . . . . . . . . . . . . . . . . . . . . . . . . . 48

4.4 Effect of Combustion Chamber Size . . . . . . . . . . . . . . 42

4.4.1 Constant Percent Fill Volume . . . . . . . . . . . . . 42 
4.4 .2 Bubble Collapse . . . . . . . . . . . . . . . . . . . . . . . 43

4.4 .3 Constant Volume . . . . . . . . . . . . . . . . . . . 46

4.5 Consistency of Bandwidth . . . . . . . . . . . . . . . 48

4.5.1 Air-Hydrogen Mixture . . . . . . . . . . . . . . . . 48

4.5.2 Oxygen-Hydrogen Mixture . . . . . . . . . . . . . 50

4.5.3 Oxygen-Hydrogen Mixture and Explosive Charge Spectrum

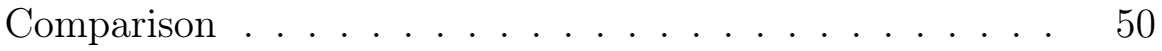

4.6 Repeatability . . . . . . . . . . . . . . . . . . . . . . . 52

4.6.1 Times Series Cross-Correlation _. . . . . . . . . . 53

4.6.2 Band Limited Cross-Spectral Analysis . . . . . . . . . . 55

4.6 .3 Band-Limited ESL . . . . . . . . . . . . . . . 58

$\begin{array}{lll}\text { Chapter } 5 & \text { Apparatus Robustness } & 61\end{array}$

5.1 Threaded Connections . . . . . . . . . . . . . . . . . . . 61

5.2 Solenoid Valves . . . . . . . . . . . . . . . . . . . . . . . 64

5.3 Flame Arrestor . . . . . . . . . . . . . . . . . . . . 65

5.4 Water Level Sensor . . . . . . . . . . . . . . . . . 66

$5.5 \quad$ Spark Plugs . . . . . . . . . . . . . . . . 67

$\begin{array}{lll}\text { Chapter } 6 & \text { Conclusions } & 68\end{array}$

$\begin{array}{lll}\text { Appendix A Water level sensor } & \mathbf{7 0}\end{array}$

$\begin{array}{lll}\text { Appendix B } & \text { LabView flow charts } & 71\end{array}$

$\begin{array}{lll}\text { Appendix C CSS Time Series and Spectra } & \mathbf{7 9}\end{array}$

C.1 Air-Hydrogen, 24" Combustion Chamber . . . . . . . . . . . . 79

C.2 Air-Hydrogen, 36" Combustion Chamber . . . . . . . . . . 87

C.3 Air-Hydrogen, 48" Combustion Chamber . . . . . . . . . . . . . 96

C.4 Oxygen-Hydrogen, 48" Combustion Chamber . . . . . . . . . 101

Appendix D ESL Histograms of Deviation from the Mean 105

$\begin{array}{ll}\text { Bibliography } & 111\end{array}$

$\begin{array}{ll}\text { Vita } & 113\end{array}$ 


\section{List of Symbols}

$p(1 \mathrm{~m}, t)$ : the pressure of the measured time series data normalized to 1 meter from the source, in units of Pascals $[\mathrm{Pa}]$

$T$ : the time between the beginning and end of $p(1 \mathrm{~m}, t)$, in units of seconds [s]

$f$ : frequency, in units of Hertz $[\mathrm{Hz}]$

$j: \sqrt{-1}$

$P(f)$ : the Fourier transform of $p(1 \mathrm{~m}, t)$, in units of Pascals per seconds $[\mathrm{Pa} / \mathrm{s}]$

$1 \mu \mathrm{Pa}$ : standard reference pressure used in underwater acoustics $\left[10^{-6} \mathrm{~Pa}\right]$

$P_{\text {peak }}(1 \mathrm{~m})$ : the maximum pressure reached by the signal $p(1 \mathrm{~m}, t)$, in units of Pascals $[\mathrm{Pa}]$

$P_{\text {peak, } \mathrm{dB}}$ : the maximum pressure reached by the signal $p(1 \mathrm{~m}, t)$ referenced to $1 \mu \mathrm{Pa}$, [dB re $1 \mu \mathrm{Pa} @ 1 \mathrm{~m}$ ]

$p_{\text {rms }}(1 \mathrm{~m})$ : the root-mean-square of the signal $p(1 \mathrm{~m}, t)$, in units of Pascals $[\mathrm{Pa}]$

SPL: sound pressure level of signal $p(1 \mathrm{~m}, t)$, [dB re $1 \mu \mathrm{Pa} @ 1 \mathrm{~m}$ ]

ESL: energy source level of signal $p(1 \mathrm{~m}, t),\left[\mathrm{dB}\right.$ re $\left.1 \mu \mathrm{Pa}^{2} \cdot \mathrm{s} @ 1 \mathrm{~m}\right]$

ESD: energy spectral density of signal $p(1 \mathrm{~m}, t),\left[\mathrm{dB}\right.$ re $1 \mu \mathrm{Pa}^{2} \cdot \mathrm{s} / \mathrm{Hz} @ 1 \mathrm{~m}$ ]

$x(t)$ or $y(t)$ : arbitrary signal in the time domain

$X(f)$ or $Y(f)$ : Fourier transform of $x(t)$ or $y(t)$

$X^{*}(f)$ : complex conjugate of $X(f)$

$S_{x y}(f)$ : cross-spectral density of signals $X(f)$ and $Y(f)$

$R_{x y}(\tau)$ : cross-correlation function of signals $x(t)$ and $y(t)$

$\rho_{x y}$ : normalized form of $R_{x y}(\tau)$ where maximum value is unity

$m_{x}$ or $m_{y}$ : mean value of signals $x(t)$ or $y(t)$

$\sigma_{x}$ or $\sigma_{y}$ : standard deviation of signals $x(t)$ or $y(t)$

$\tau$ : arbitrary time delay constant, in units of seconds [s] 


\section{Chapter 1}

\section{Introduction}

This thesis describes the development and testing of the Combustive Sound Source (CSS), which is a broadband underwater sound source. Development of the CSS is being undertaken to provide an alternative to underwater explosive charges. Explosive charges used in underwater acoustic scientific research are convenient and useful, but they present inherent danger to the scientists and technicians that work with them, and are also potentially dangerous to marine life. Further, they expel irretrievable debris into the water column upon use. The CSS however, is a clean, safe, reliable, and cost-effective replacement to explosive sound sources, and offers the user an adjustable source level, while maintaining a wide bandwidth. If an electrolytic cell is used to provide the combustion gases, then no energetic materials are stored or transported. The gases are only generated at the time of the event. Hence the CSS is inherently safer to use than explosives. In Chapter 1, the history of the CSS, its basic operation, and the purpose of the present work is described. 


\section{$1.1 \quad$ Background}

Low frequency, broadband underwater sound sources are needed to conduct acoustic research in the fields of acoustical oceanography, natural resource exploration, and sonar applications, among others. These sources must be able to generate a sufficiently powerful, low frequency signal to maintain a sufficient signal to noise ratio (SNR) at the receiver over large source-receiver range scales [1]. It is also desired that these sources be broadband to gather data on the dispersion effects and the nonlinear frequency dependence of ocean sediments as described in Holmes [2] and Knobles [3].

Small explosive charges are popular due to their broad bandwidth, high energy density, ease of deployment, and low cost [4]. However, the hazards innate in explosive charges have led to the development of alternative sound sources. Ideally, these alternative sound sources should be capable of producing a signal that maintains the wide bandwidth of explosive charges with reduced amplitude to satisfy environmental concerns. Sound sources such as low frequency shakers [5] and resonators [6] have been developed, but often do not have the bandwidth, convenience, and efficiency of explosive charges. A number of other sources are described in Dobrin [7], but the CSS differs from all of these, and is more similar to an explosive source in the following manner: The CSS generates pressure directly in the water, and no energy is lost through motion of an elastic membrane, as in the Aquapulse (trademark of Western Geophysical Co.) or through the motion of any mechanical piston or valve as in sparkers or boomers [7]. Hence the CSS is more efficient in the conversion of stored energy into acoustic energy, and this conversion can be achieved with the required bandwidth. 


\subsection{Literature Review}

A typical CSS consists of a submersible combustion chamber, open to the water filled with a combustible fuel/oxidizer mixture. The mixture is ignited and a combustion wave propagates through the mixture. During this process the fuel and oxidizer are converted into high temperature combustion products. The temperature increase occurs sufficiently quickly as to cause an increase in pressure, which in turn, causes the bubble to expand. The initial pressure pulse is radiated to the far field, and while the bubble grows larger than the original volume, a negative acoustic pressure is radiated. At some point, a maximum volume is reached and the bubble begins to collapse. At the minimum bubble volume, which is significantly less than the original volume, a sharp, positive transient acoustic pulse is radiated. This process repeats until the energy is expended, or until the bubble either breaks apart or the combustion products condense (which is the case for stoichiometric hydrogen/oxygen combustion). The expansion and collapse of the bubble is the main source of radiated sound. Although never fully developed, the device was patented in 1924 [8], and since that time, several papers and patents regarding the concept of the CSS have been published, such as Anderson [9], Owen [10], and Wilson [11, 12], among others.

\subsubsection{The Combustion Process}

There are two classes of combustion, known as deflagration and detonation. The CSS utilizes deflagration, which is a subsonic combustion process whereby a thermal wave travels through the combustive mixture raising the temperature of the mixture by thermal conduction to the point of ignition. This requires an appropriate fuel-oxidizer mixture and an ignition event, such as a spark. Detonation 
is a supersonic combustion process by which a shock wave propagates through the combustive mixture due to an initial energy release. The compression of the combustive mixture due to the propagation of the shock wave increases the local temperature to a level high enough to initiate ignition. Although both classes of combustion require an appropriate fuel-oxidizer mixture, the detonation process does not require a spark to initiate combustion. In general, detonations are more destructive than deflagrations due to the associated high pressures of the shock wave; however, deflagrations can be made more controllable due to the reliance on an ignition source and the subsonic propagation speeds of the combustive process [13].

It should be emphasized that the CSS is not an explosive source. Quoting Glassman and Yetter [14]:

It is a very common error to confuse a pure explosion and a detonation. An explosion does not necessarily require the passage of a combustion wave through the exploding medium, whereas an explosive gas mixture must exist in order to have either a deflagration or a detonation. That is, both deflagrations and detonations require rapid energy release; but explosions, though they too require rapid energy release, do not require the presence of a waveform.

The central feature of explosives is that the reaction rate of an explosive mixture varies significantly as a function of temperature and pressure. At low temperatures and pressures the reaction rate of an explosive is low. However, when the temperature or pressure is raised to a sufficient level a violent exothermic reaction takes place throughout the explosive instantaneously, without the need of a prop- 
agating wave as in combustion. A deflagration combustion process is therefore less violent and is easier to control than a detonation or explosion.

\subsubsection{Parametric Analysis of Gas Volume, Ignition Depth, and Gas Mixture}

A major focus of the literature relating to CSS analysis involves three parameters controlling the acoustic signature of any combustive sound source: gas volume, ignition depth, and gas mixture. It has been difficult for researchers to isolate the interdependencies of these parameters, but qualitative results for each case have been published. Wilson et al. [11, 12] tested these parameters using methane and oxygen. A conical combustion chamber was used to provide a constant aspect ratio (height to diameter) for various volumes of gas. The effect of varying total gas volume at a stoichiometric equivalence ratio, varying the depth of ignition, and varying the equivalence ratio for a constant volume of fuel at a set depth were studied.

Wilson et al. [12] showed that altering the total gas volume for a given equivalence ratio affects the period of the initial bubble pulse, which is determined from the time between initiation of bubble growth and the first bubble collapse. It was found that the period of this bubble pulse increases with increasing gas volume.

It was also found that altering the depth of ignition affects the period of the initial bubble pulse. Wilson et al. [12] investigated this depth dependence for both constant mass and for a constant physical volume of gas. For constant mass and constant physical volume, the period of the first bubble pulse was found to decrease with depth. This implies that the depth of ignition has a larger effect on

the initial bubble period than the energy of combustion of the gas, which is also supported by the Rayleigh-Willis equation given in Eq. 1.18 and discussed below. 
The effect of varying the equivalence ratio with a constant volume of fuel was also investigated in Ref. [12] where the volume of methane remained constant, while the volume of oxygen was altered to obtain the desired equivalence ratio. It was found that the bubble periods decreased throughout the experiment as the equivalence ratio increased. This implies that the bubble period depends more on total gas volume than the total fuel volume.

Wilson et al. [12] experimented with several combustion chamber shapes, fueloxidizer mixtures, and ignition methods to determine the optimum means of generating high intensity, low frequency signals. Cylindrical, conical, and hemispherical combustion chambers were tested. Although an equivalent volume of gas was used for each case, it was determined that the conical combustion chamber yielded the highest radiated peak pressure. Hydrogen and methane were both tested as fuels, and oxygen and air were both tested as the oxidizer in the combustive mixture. It was found that a hydrogen-oxygen mixture yields the highest acoustic output. A single spark was compared to four $\frac{1}{4}$-strength simultaneous sparks as the ignition source. It was found that the acoustic output of the four spark ignition system was around $20 \mathrm{~dB}$ higher across the entire bandwidth. Therefore, Wilson et al. [12] concluded that having a conical combustion chamber, a hydrogen-oxygen combustive mixture, and multiple ignition sources would yield a higher acoustic output than the other tested methods.

\subsubsection{Versatility}

It has also been shown that the CSS is a versatile acoustic energy source that can easily be customized to perform in various configurations and environments [15]. In addition to a stationary deployment in the water column, a CSS can be de- 
ployed underwater in a tow body and as an array of sources. It has also been demonstrated that a CSS is capable of producing seismic interface waves for both air-earth and water-seafloor interfaces. Measured energy levels and spectra from a CSS were compared to that of similar sources used for each medium. These tests confirm the versatility and cost efficiency of deployment for each configuration [16].

\subsection{Purpose of Work}

A desire for safety features was derived from the inherent hazardous nature of underwater explosive charges. The Naval Air Systems Command (NAVAIR) manual warns, "It is possible that firing will occur on release of the arming piston. This can happen during a ship sinking and cause other adjacent explosives to detonate, resulting in injury to surviving personnel in the water [17]." Furthermore, past misuse of underwater explosive charges has led to the death of at least two individuals [18]. This led to the temporary ban of underwater explosive charges and initiated CSS research $[11,12,16]$. Recent improvements to the CSS provide inherent safety features as well as a minimal duty cycle to reduce the hazardous nature of the device.

The present development of the CSS was undertaken to provide a safe, reliable efficient replacement to underwater explosive charges as a source of acoustic energy. This entails that the CSS is capable of producing an acoustic output with similar spectral characteristics at an amplitude approaching that of an underwater explosive charge comprised of $1.8 \mathrm{lbs}$ of TNT, as tabulated by Chapman [4] and modeled by Wakeley [19]. As a safety feature, it was desired that no energetic material be transported or stored to operate the CSS. Previous versions of the CSS failed to meet these needs. Analysis of the previous work $[11,12,16]$ yielded 


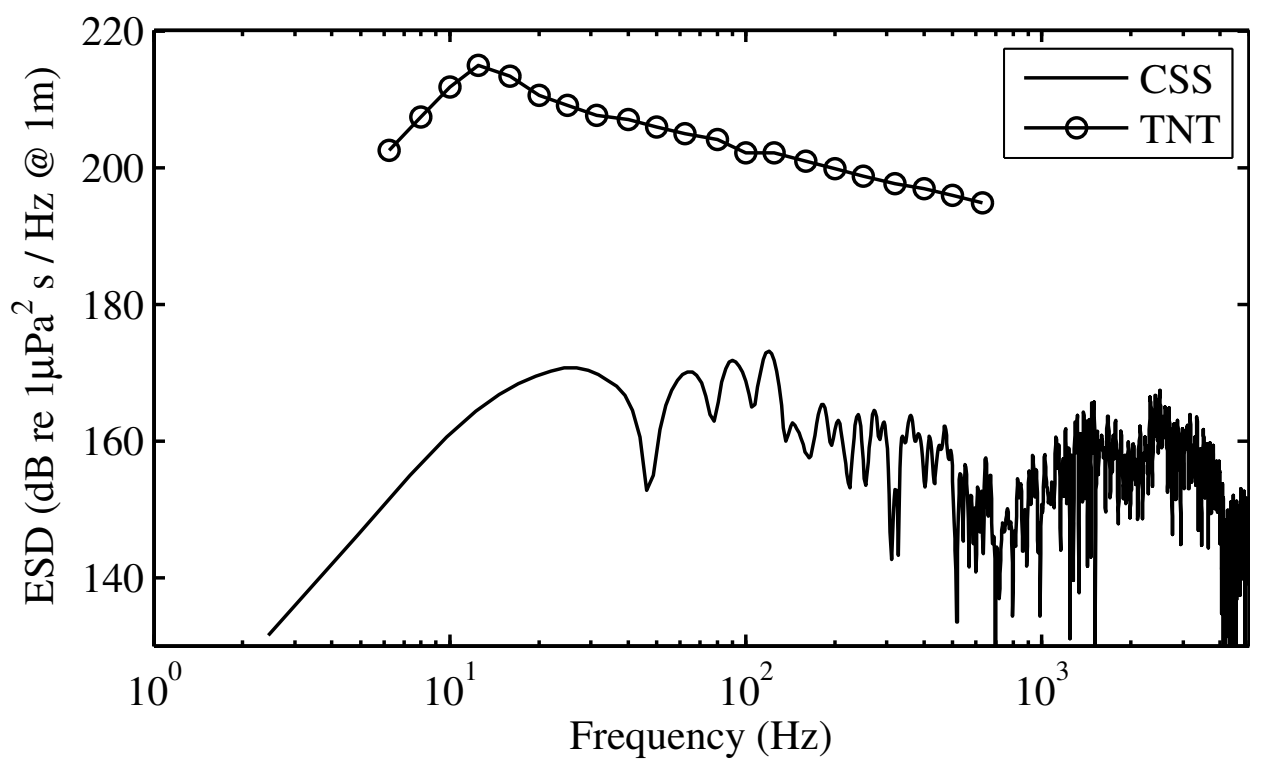

Figure 1.1: ESD of 1.8lb TNT explosive charge charge fired at $23 \mathrm{~m} \mathrm{[4],} \mathrm{and} \mathrm{CSS} \mathrm{event} \mathrm{of}$ approximately $6 \mathrm{~L}$ (STP) of hydrogen and oxygen mixture fired at $25 \mathrm{~m}$.

insight into improvements that were made, which allowed the CSS to more closely meet the desired goals.

Wilson et al. [12] did not achieve the source level (SL) currently desired, but they found that using hydrogen and oxygen, a conical chamber, and multiple ignition sources all increased the source level. The spectrum of a previous CSS shot ${ }^{1}$ is shown to be around $40 \mathrm{~dB}$ lower than a spectrum produced by an underwater explosive charge as seen in Fig. 1.1. These conclusions were the basis of the current study, as discussed in Chapter 2.

\subsection{Source Level Metrics}

The source level (SL) for a transient acoustic signal can be quantitatively described by four different SL metrics: peak pressure, sound pressure level (SPL), energy source level (ESL), and energy spectral density (ESD). These metrics were used

\footnotetext{
${ }^{1}$ Obtained by Preston Wilson of ARL:UT during SW06 experiment on August 25, 2006 at 10:59 a.m.
} 
to analyze the data reported in Chapter 4. Each measured acoustic pressure time series, $p(1 \mathrm{~m}, t)$, will refer to the acoustic pressure of the signal measured in the far field and amplitude normalized through spherical spreading to 1 meter from the source. The peak pressure level is the maximum pressure reached in a signal defined by

$$
P_{\text {peak }, \mathrm{dB}}=20 \log _{10} \frac{P_{\text {peak }}(1 \mathrm{~m})}{1 \mu \mathrm{Pa}} \quad[\mathrm{dB} \text { re } 1 \mu \mathrm{Pa} @ 1 \mathrm{~m}],
$$

where

$$
P_{\text {peak }}(1 \mathrm{~m})=\max (|p(1 \mathrm{~m}, t)|) .
$$

The sound pressure level (SPL) is

$$
\mathrm{SPL}=20 \log _{10} \frac{p_{\mathrm{rms}}(1 \mathrm{~m})}{1 \mu \mathrm{Pa}} \quad[\mathrm{dB} \text { re } 1 \mu \mathrm{Pa} @ 1 \mathrm{~m}],
$$

where

$$
p_{\text {rms }}(1 \mathrm{~m})=\sqrt{\frac{1}{T} \int_{0}^{T} p^{2}(1 \mathrm{~m}, t) d t},
$$

and $T$ is the total time of the signal.

The energy source level (ESL) is

$$
\mathrm{ESL}=10 \log _{10} \frac{\int_{0}^{T} p^{2}(1 \mathrm{~m}, t) d t}{1 \mu \mathrm{Pa}^{2} \cdot \mathrm{s}} \quad\left[\mathrm{dB} \text { re } 1 \mu \mathrm{Pa}^{2} \cdot \mathrm{s} @ 1 \mathrm{~m}\right]
$$

where $T$ is the total time of the signal. 
The energy spectral density (ESD) is

$$
\operatorname{ESD}(f)=10 \log _{10} \frac{|P(f)|^{2}}{(1 \mu \mathrm{Pa} \cdot \mathrm{s})^{2}} \quad\left[\mathrm{~dB} \operatorname{re} 1 \mu \mathrm{Pa}^{2} \cdot \mathrm{s} / \mathrm{Hz} @ 1 \mathrm{~m}\right],
$$

where $f$ is frequency and $P(f)$ is taken as the Fourier transform of $p(1 \mathrm{~m}, t)$, defined by

$$
P(f)=\int_{-\infty}^{\infty} p(1 \mathrm{~m}, t) \exp (j 2 \pi f t) d t
$$

for $-\infty \leq f \leq \infty$.

When reporting ESD as a function of frequency one has three basic choices. The first option is to display ESD as calculated above for both positive and negative frequencies. This option is unambiguous, but it is difficult to display the negative frequencies in a logarithmic fashion, which is common when frequency is plotted on the horizontal axis. The second option is to display ESD for only the positive frequencies without making any adjustment to account for the energy contained in the negative frequencies which are not being displayed. This option allows frequency to be displayed in a logarithmic fashion, but it does not account for the energy being ignored in the negative frequency bands. The third option is to display ESD for only the positive frequencies and adjust the levels to account for the energy in the negative frequencies, which is not being displayed. This option allows frequency to be displayed in a logarithmic fashion, and it accounts for the total energy in the positive and negative frequencies. In this option the ESD will be $3 \mathrm{~dB}$ higher than the discrete calculation of ESD given in the above equations. In this thesis, all figures and values reported for ESD are given as the latter option to display frequency on a logarithmic scale and account for the energy in both the 


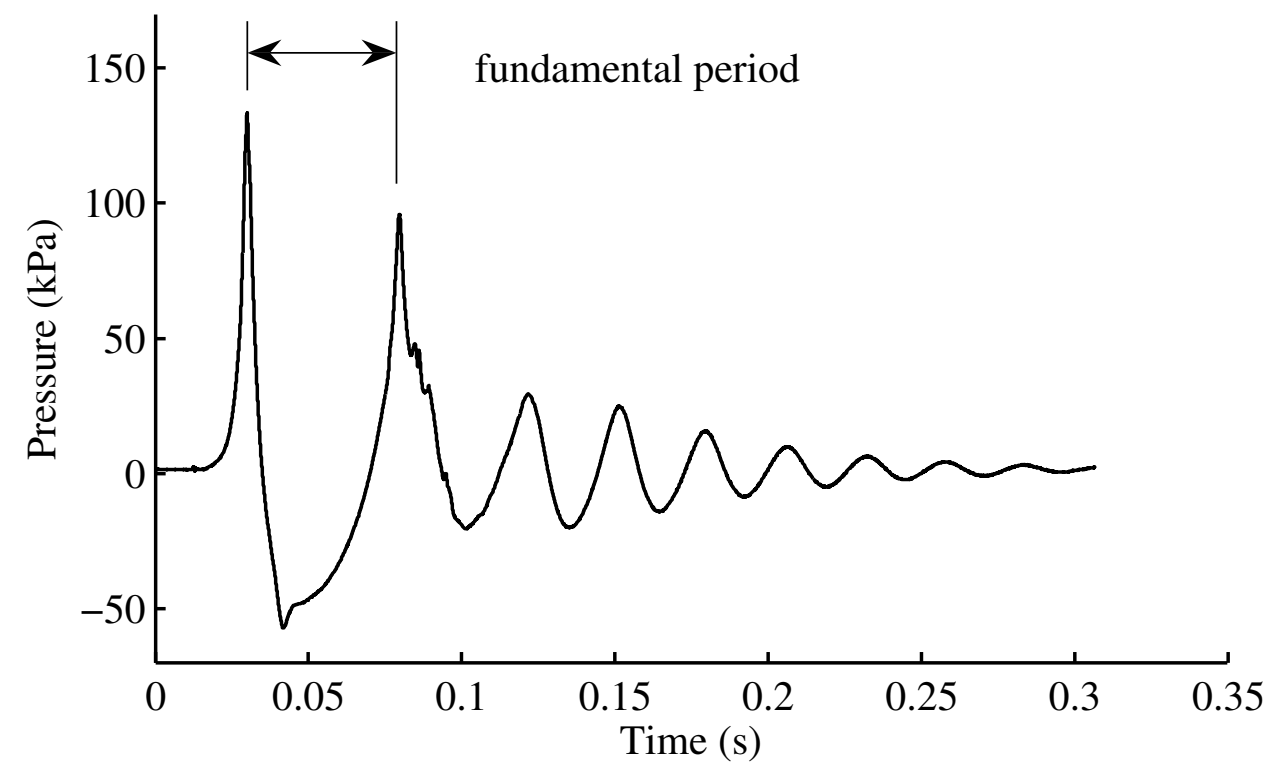

Figure 1.2: A time series of a CSS event is given, where the time between the first two peaks is shown as the fundamental period.

positive and negative frequencies.

Additional metrics were used to further analyze the collected data. The fundamental period of a CSS event is the time between the first and second peaks in the time series, respectively, as shown in Fig. 1.2. The fundamental frequency is given by the inverse of the fundamental period, and it can also be determined from the ESD as it is the lowest frequency peak on an ESD curve, as seen in Fig. 1.3.

Similarly, other metrics were used to describe the repeatability and differences of various signals. The fundamental frequency and the ESD at the fundamental frequency were analyzed to determine how the frequency spectrum is altered for a given parameter change. Band limited ESL calculations were used to determine how various bands are affected by a parameter change. The peak cross-correlation value was used to quantify the repeatability of events in the time domain, and cross-spectral analysis was used to quantify the repeatability over octave bands in the frequency domain. Each of these additional metrics yields further insight into 
the repeatability and resultant trends of the parametric adjustments throughout the experiment.

The cross-correlation for two signals, $x(t)$ and $y(t)$, is

$$
\rho_{x y}=\left|\frac{R_{x y}-m_{x} m_{y}}{\sigma_{x} \sigma_{y}}\right|,
$$

where $m_{i}$ is the mean of the $i$ th signal in the time domain, $\sigma_{i}$ is the standard deviation of the signal in the time domain, and

$$
R_{x y}(\tau)=\lim _{T \rightarrow \infty} \frac{1}{T} \int_{-T / 2}^{T / 2} x(t) y(t+\tau) d t .
$$

This limits $\rho_{x y}$ to a minimum of zero, corresponding to no correlation, and a maximum of one, corresponding to an exact correlation. The cross-spectral analysis values were calculated as

$$
R_{x y}(\tau)=\int_{-\infty}^{\infty} S_{x y}(f) \exp (j 2 \pi f \tau) d f,
$$

where

$$
S_{x y}(f)=\frac{X^{*}(f) Y(f)}{T},
$$

and $X(f)$ and $Y(f)$ are the Fourier Transform pairs of $x(t)$ and $y(t)$, respectively. Here $X^{*}(f)$ denotes the complex conjugate of $X(f)$. Similarly, $R_{x y}$ can be normalized to a maximum of unity using Eq. 1.8.

\subsection{Rayleigh-Willis Bubble Model}

A decrease in the fundamental frequency should be expected for events with increasing gas volume on the basis that it will take a longer time for the bubble to reach the maximum point of expansion and ultimately collapse for large volumes 


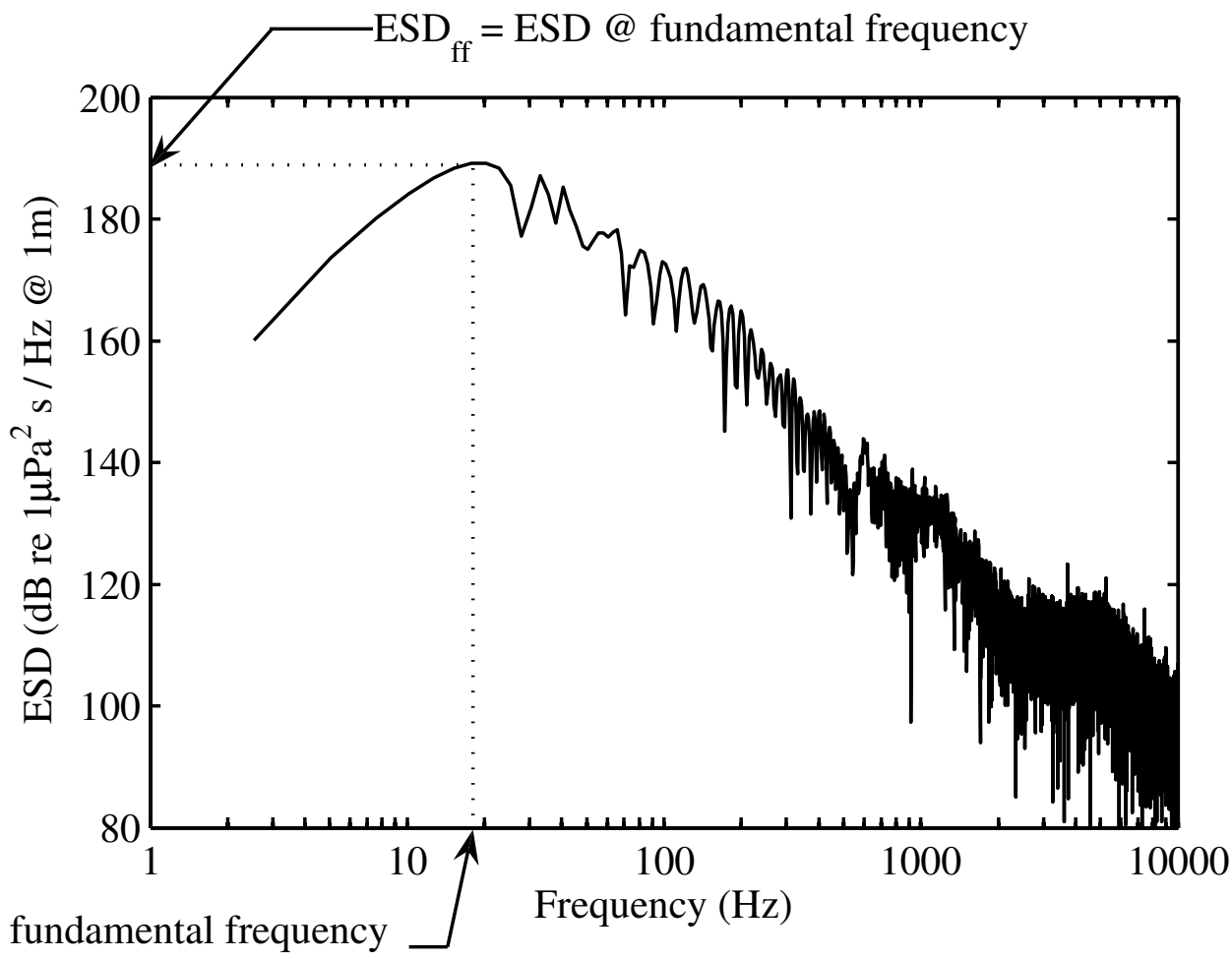

Figure 1.3: The fundamental frequency and ESD at the fundamental frequency of a CSS event.

of gas. This increase in time creates a longer fundamental period, and thus a lower fundamental frequency. This trend is supported by Rayleigh's equation describing the period of collapse for a vapor bubble or void given by [20]

$$
T=1.83 A_{m} \sqrt{\frac{\rho}{P_{0}}}
$$

where $T$ is the period of bubble oscillation, in seconds, $A_{m}$ is the maximum radius of the bubble, in meters, $\rho$ is the density of the surrounding fluid, in kilograms per cubic meter, and $P_{0}$ is the hydrostatic pressure, in Pascals. Equation 1.12 shows that an increase in bubble radius directly corresponds to an increase in bubble 
period. Thus, a reduction in the fundamental frequency occurs for larger volumes of gas.

Equation 1.12 also predicts a decrease in the period of bubble collapse, or equivalent increase in fundamental frequency, for an increase in ambient hydrostatic pressure. It can be seen that Eq. 1.12 predicts the fundamental frequency to be more sensitive to depth than volume when recast in terms of bubble volume rather than bubble radius. Given,

$$
A_{m}=\sqrt[3]{\frac{3 V}{4 \pi}},
$$

where $V$ is the bubble volume, Eq. 1.12 can be written as

$$
T=1.83 \sqrt[3]{\frac{3 V}{4 \pi}} \sqrt{\frac{\rho}{P_{0}}} .
$$

Therefore, a proportionality can be developed between bubble period, bubble volume, and hydrostatic pressure at ignition depth:

$$
T \propto \frac{V^{1 / 3}}{P_{0}^{1 / 2}} .
$$

This equation shows that the fundamental bubble period is more sensitive to hydrostatic pressure than bubble volume, which agrees with Wilson et al. [12] and the results of the present work discussed in Chapter 4 .

It is also useful to describe the bubble period in terms of the potential energy of the bubble. A relation between bubble pressure, volume, and potential energy was found by Willis [21] in 1941, 


$$
Q=\frac{4}{3} \pi A_{m}^{3} P_{0}
$$

where $Q$ is the potential energy of the bubble. A relationship between $T$ and $Q$ can be obtained by solving Eqs. 1.12 and 1.16 for $A_{m}$ as follows:

$$
T=1.14 \rho^{1 / 2} P_{0}^{-5 / 6}(K Q)^{1 / 3},
$$

where $K$ is a constant with a value dependent upon the units of $Q\left(K=10^{10}\right.$ if $Q$ is in kilojoules). However, in SI units with $1024 \mathrm{~kg} / \mathrm{m}^{3}$ as the density of seawater and $P_{0}=\rho g(d+10.1)$, where $d$ is the depth of the bubble center, and $g$ is the acceleration of gravity, Eq. 1.17 can be given as,

$$
T=\frac{0.1673 Q^{1 / 3}}{(d+10.1)^{5 / 6}},
$$

which is the classic Rayleigh-Willis equation [21]. Equation 1.18 shows that an increase in bubble period should be expected from an increase in the potential energy of the combustive mixture and decrease in ignition depth.

\subsection{Thesis Organization}

Proceeding from the aforementioned considerations led to the development of a new CSS that would provide an increased source level and the robustness to perform various experiments. The details of the new design are given in the following chapter. Chapter 2 also discusses the essential hardware components needed for any CSS and the method by which they were implemented in the current design. 
Other convenient features added to the current CSS are also discussed. Chapter 3 gives a description of the experiment which tested the acoustic output and robustness of the new CSS for various parameters. Chapter 4 discusses the results of this experiment. The effect of gas volume, ignition depth, gas chemistry, and combustion chamber size are all discussed. The repeatability of the new CSS is also quantified in the time and frequency domains. Chapter 5 discusses the robustness of the apparatus during the experiment. Finally, Chapter 6 provides a conclusion. 


\section{Chapter 2}

\section{Description of Apparatus}

There are three essential hardware components of a repeatable, reusable CSS: a gas delivery system, a submersible chamber to entrap gas below the surface of the water, and an ignition source. A repeatable gas delivery system is needed to allow users to accurately deliver the desired amounts of fuel and oxidizer into the submersible chamber before firing the CSS. The chamber must be capable of withstanding the forces and shock loading applied by the combustion process and subsequent bubble activity at a range of depths. Failure of the chamber to withstand high levels of shock loading will quickly result in cracks and leaks. An ignition source within the chamber is needed to initiate the combustion process. This ignition source must be repeatable to ensure that the combustion event is initiated in the same manner over long periods of use. A schematic of these components along with the electronic controls and a recording system is presented in Fig. 2.1.

\subsection{Combustion Chamber}

A submersible chamber is required to entrap gas below the water surface during the combustion process. Four conical chambers were built with base diameters 


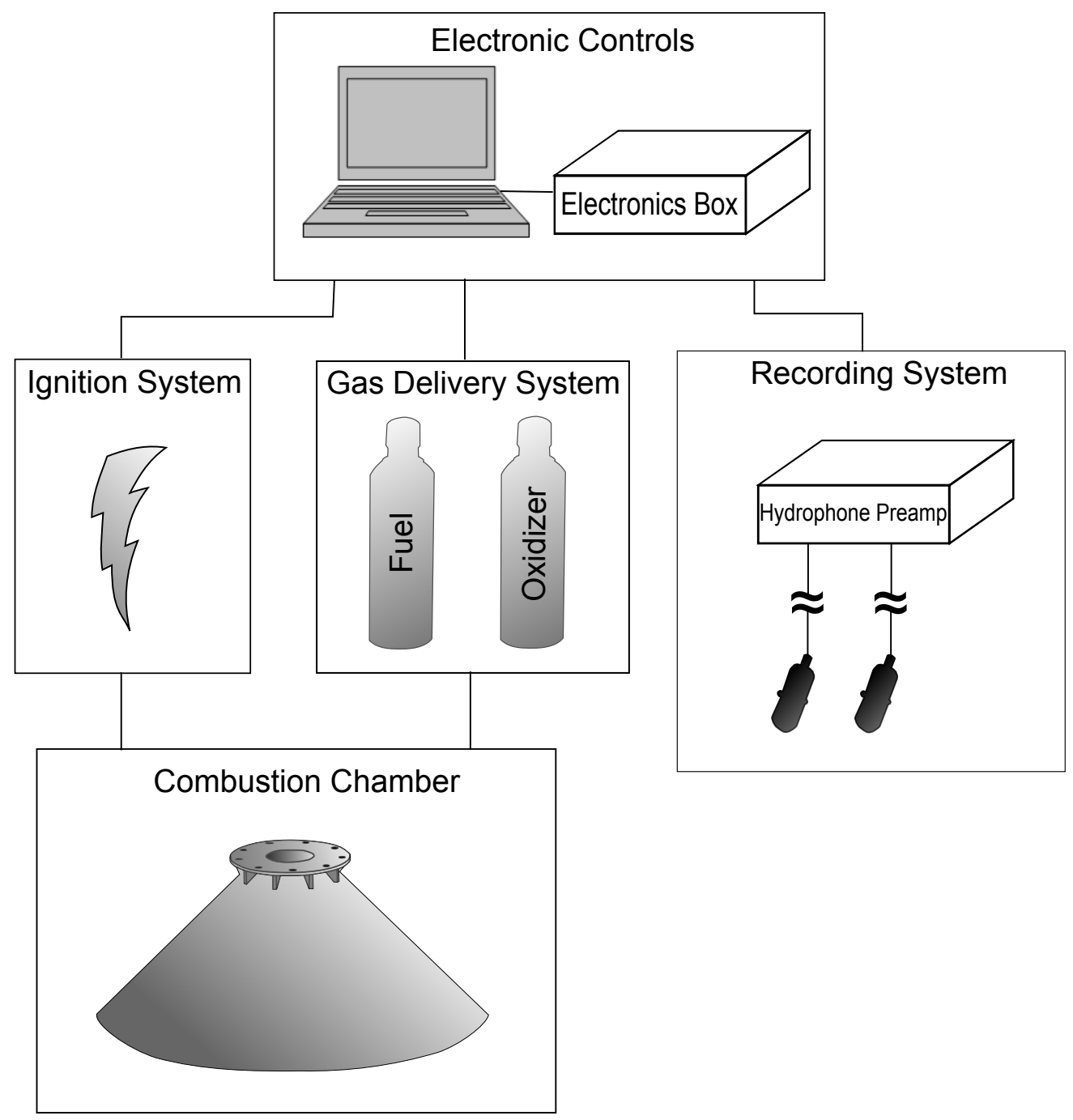

Figure 2.1: A general schematic for the relation between electronic controls, ignition system, gas delivery system, combustion chamber and recording system for the CSS.

of $12^{\prime \prime}, 24^{\prime \prime}, 36^{\prime \prime}$, and 48". The sides of the chambers create a $45^{\circ}$ angle with the horizontal, and have total volumes of 3.7 L, 29.6 L, $100 \mathrm{~L}$, and $237 \mathrm{~L}$, respectively. Each chamber is made of $\frac{1}{2}$ " thick powder coated A36 steel to ensure strength and resistance to corrosion. Each chamber is open on the bottom and cut short of the apex so as to leave the chamber open on top. A circular flange was welded around the top of each chamber opening via gussets. Bolts through this flange 
allow the cones to individually be attached and removed from the ignition and gas delivery system. Ballast weights were welded to the bottom edge of the combustion chamber to overcome buoyancy when filled with gas. The combustion chamber with a 48 " base diameter is shown in Fig. 2.2.

The combustion chambers were designed to have a conical shape due to previous findings that a conical combustion chamber yielded a greater acoustic output, as described in Section 1.2.2. Multiple chamber sizes of increasing volume were desired for versatility, and it was desired that the largest chamber have the capacity to contain a volume of gas for which the potential energy of combustion was equivalent to the energy released by an underwater explosive charge. It was determined that building four combustion chambers with base diameters of 12", 24", 36", and 48" would be cost efficient, manageable, and yield the versatility to scale the volume of gas, and with potential energy approaching that of an underwater explosive charge. The $1 / 2^{\prime \prime}$ thick A36 steel was found to be the most cost efficient material that could withstand repeated CSS use and prevent fatigue,

and the powder coating was added to prevent corrosion for use in water. The combustion chambers were designed to withstand a cyclic stress limit of 34,000 psi from the prediction that each chamber could experience a cyclic stress limit up to 17,000 psi, which yields a design safety factor of 2 .

\subsection{Gas Delivery System}

As seen in Fig. 2.3, the gas delivery and ignition system are primarily comprised of a three tiered tower bolted on top of a combustion chamber and separated by a rubber gasket to prevent leakage. The bottom tier consists of a combustion chamber head and flange held directly above the chamber. This head piece con- 


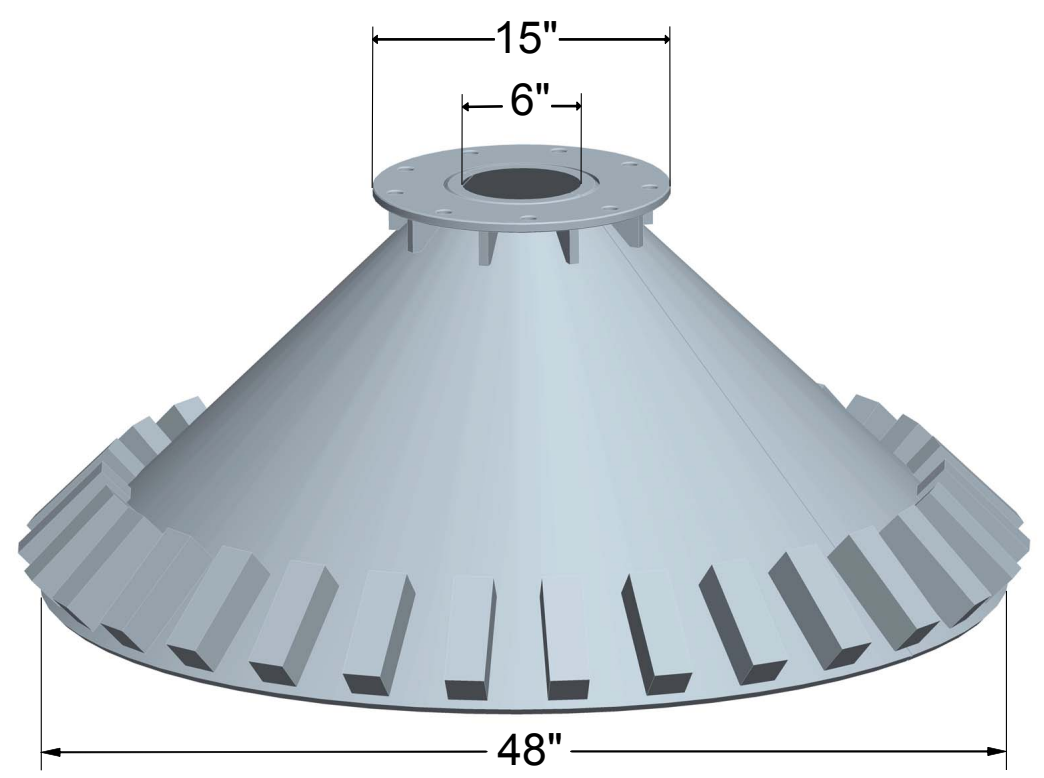

Figure 2.2: A representation of the combustion chamber with base diameter of 48". Drawing made by Richard Lenhart, The Applied Research Laboratories, The University of Texas at Austin.

tains several ports which allow spark plugs and tubing to be mounted into the combustion chamber. The middle tier consists of a gas manifold and mounting ports for stick coils used in the ignition system. On the top tier is a pressure vessel containing capacitors used to power the ignition system. Each tier plays a vital role in firing the CSS.

Although previous studies, discussed in Section 1.2.2, found the oxygen-hydrogen mixture to yield the greatest acoustic output, the current gas delivery system was designed to deliver air and hydrogen. Air was substituted for oxygen due to the dangers involved in transporting oxygen through hoses which may become contaminated with unwanted material and residual products of the gas flow. The CSS combustion chambers and ignition system are capable of firing mixtures comprised of an oxygen-hydrogen mixture, but the gas delivery system described in this section is not intended for oxygen use. A separate system will be presented in Section 3.1 that describes how an oxygen-hydrogen mixture is delivered to a 
chamber.

The gas delivery system ensures that the desired volume of each gas mixture is delivered into the combustion chamber while at depth. The system consists of separate bottles of compressed air and hydrogen, Victor ${ }^{1}$ regulators, Sierra ${ }^{2}$ mass flow controllers, Omega ${ }^{3}$ solenoid valves, appropriate hose and tubing, and Parker ${ }^{4}$ check valves. A simplified gas delivery system schematic is given in Fig. 2.4. Fifty foot hoses connect each regulator to a Sierra mass flow controller. A Parker $1 / 2^{\prime \prime}$ inner diameter (ID), 300 psi working pressure air hose was used in the air circuit, and a Parker 3/8" ID T-Grade fuel hose was used in the hydrogen circuit. The mass flow controllers allow users to specify the amount of each gas type injected into the combustion chamber through manual inputs in LabVIEW software. The outlet of the mass flow controllers are connected to 330 feet of hose. The 330 foot hose in the hydrogen circuit is then connected to a $\mathrm{SGD}^{5}$ flashback arrestor, that has a maximum rating of $150 \mathrm{psi}$, preceding a solenoid valve. The 330 foot hose in the air circuit is connected directly to a solenoid valve. The solenoid valves are mounted on the vertical supports of the three tiered tower and are controlled by LabVIEW software, which controls the opening and closing of the valves to keep the hoses pressurized to ambient conditions. It is necessary to keep the hoses pressurized to ensure that the amount of gas expelled into the chamber is equivalent to the amount of gas allowed through the mass flow controllers. Unpressurized hoses create significant uncertainty in the amount of gas expelled in the chamber. From this point downstream, the pneumatic circuits for hydrogen

1Victor Equipment Co., http://www.thermadyne.com/victor

${ }_{2}^{2}$ Sierra Instruments, Inc., http://www.sierrainstruments.com

${ }^{3}$ Omega Engineering Inc., http://www.omega.com

${ }^{4}$ Parker Hannifin Corp., http://www.parker.com

${ }_{5}^{5} \mathrm{SGD}$, Inc., http://www.sgd.com 


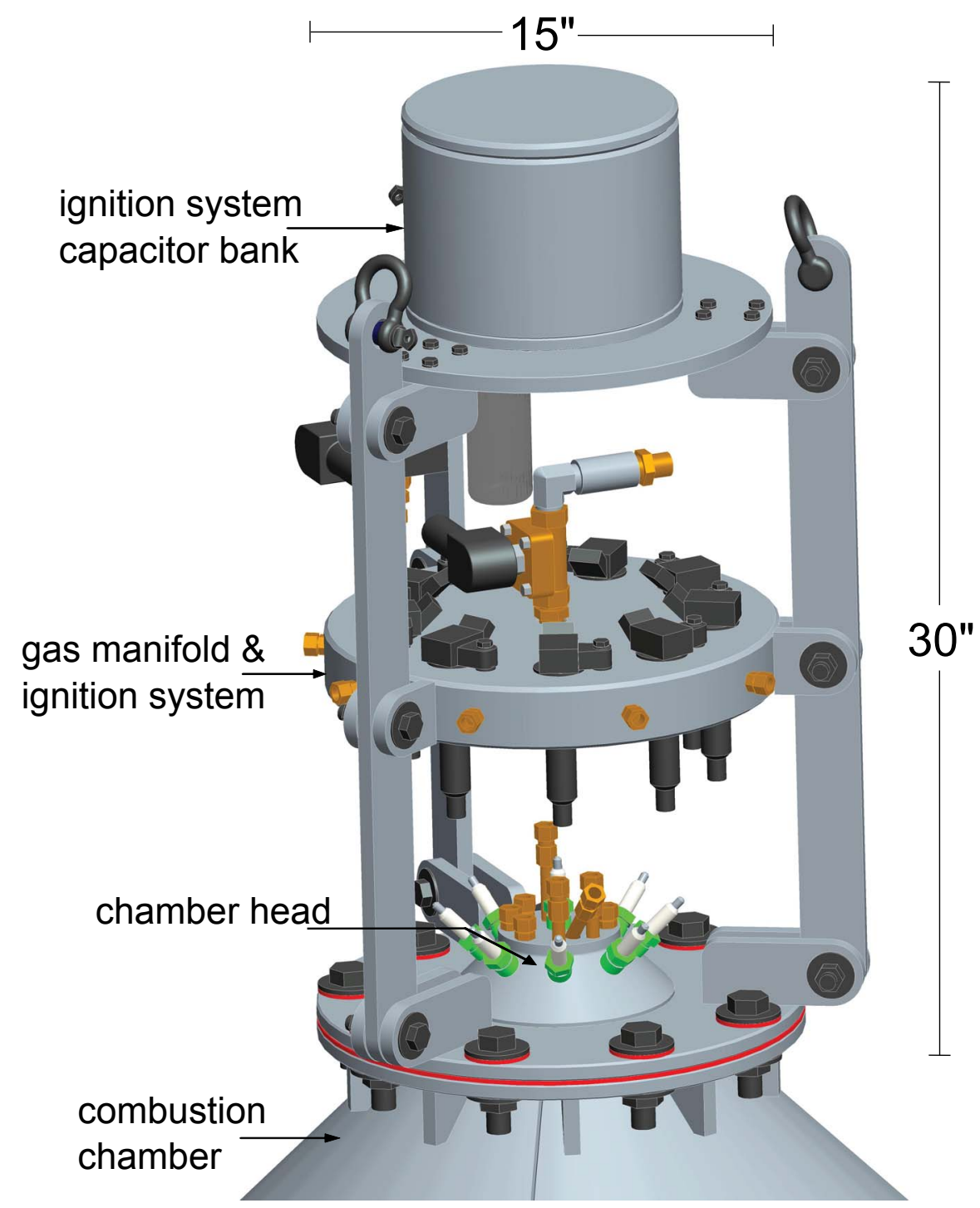

Figure 2.3: A representation of the three tiered tower used in the CSS ignition and gas delivery systems. Drawing made by Richard Lenhart, The Applied Research Laboratories, The University of Texas at Austin. 
and air are different.

The hydrogen gas is sent through the flashback arrestor and solenoid valve, then through a single tube terminated with a Parker check valve with 1 psi cracking pressure that has a maximum rating of 6000 psi. Hydrogen gas allowed through the check valve flows into the combustion chamber through the chamber head and top opening of the combustion chamber. The flashback arrestor ensures that the flame produced during the combustion event does not propagate back into the hose containing hydrogen, and the check valve prevents gas and water backflow into the pneumatic circuit before, during, and after the combustion event.

The air is sent through a solenoid valve and then divided into nine different tubes, each terminated with a Parker check valve with a 5 psi crack pressure and a maximum rating of 6000 psi. Air allowed through the check valves flows into the chamber through the chamber head and into the top of the combustion chamber. The need for nine tubes in the air delivery system is discussed along with the ignition system in the following section. The check valves terminating the air tubing serve the same purpose as that of the hydrogen circuit. It should be noted that as a safety precaution, fuel and oxidizer are not allowed to mix until reaching the combustion chamber. Once the fuel and oxidizer reach the combustion chamber a sufficient diffusion time, tabulated in Table 2.1, is allowed for a given volume before ignition.

\subsection{Ignition System}

A repeatable ignition system was used to initiate the combustion process for each event. As described in Section 1.2.2, previous work indicated that multiple-spark ignitions increased the acoustic output. It was determined that nine spark plugs 


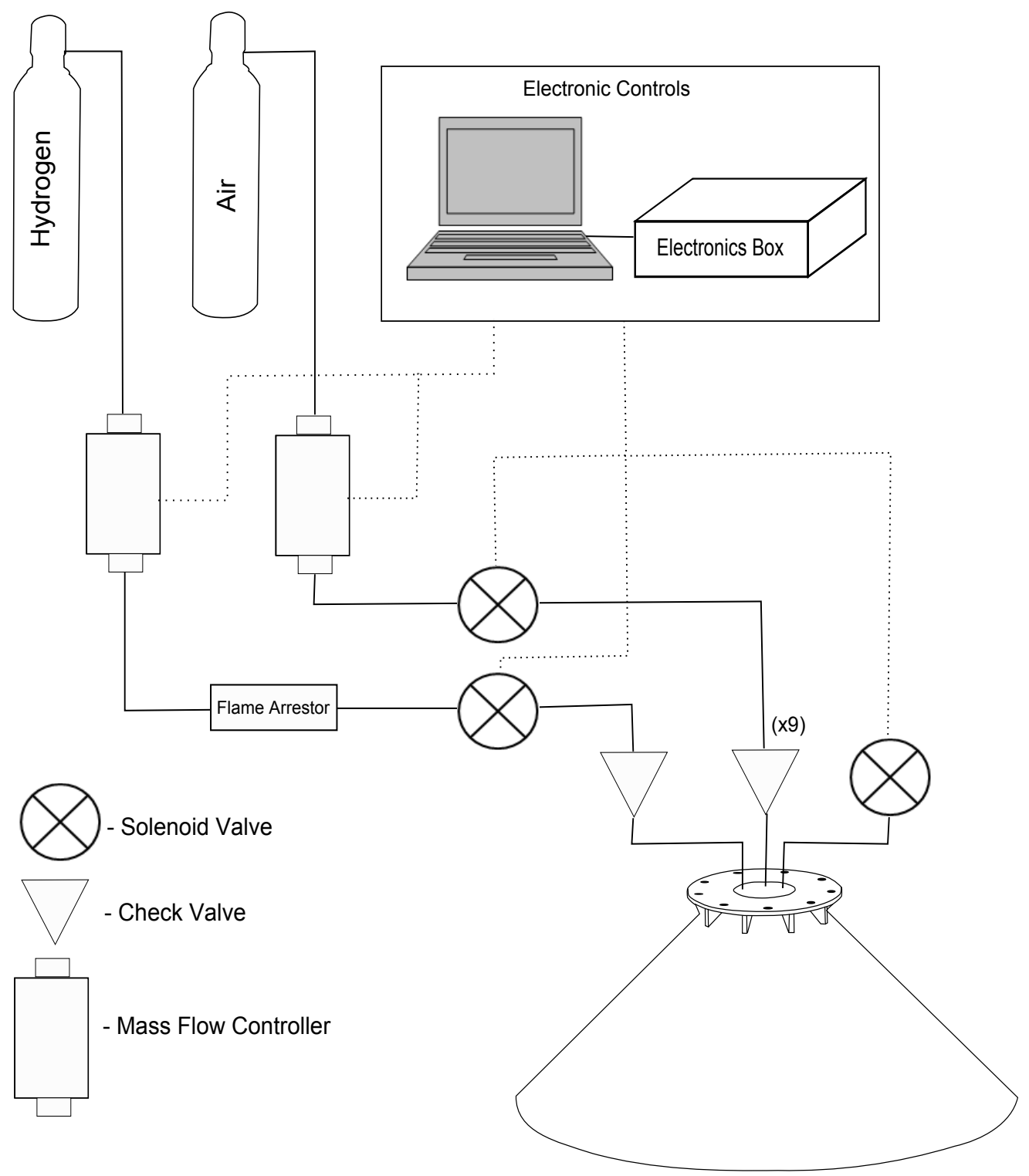

Figure 2.4: A general schematic of the gas delivery system containing gas bottles, mass flow controllers, flame arrestor, and various valves.

could be positioned around the manifold. The plugs were mounted in ports, which slightly extend into the combustion chamber. Eight plugs form a circle with the ninth plug in the middle of the circle, as shown in Fig. 2.5.

Each spark plug is connected to a stick coil from a 2005 Toyota Tundra. The 


\begin{tabular}{|r|r|r|}
\hline & $5 \mathrm{~m}$ & $10 \mathrm{~m}$ \\
\hline \hline $6 \mathrm{~L}$ & $2 \mathrm{~min}$ & $3 \mathrm{~min}$ \\
\hline $10 \mathrm{~L}$ & $2.5 \mathrm{~min}$ & $3.5 \mathrm{~min}$ \\
\hline $12 \mathrm{~L}$ & $3 \mathrm{~min}$ & $4 \mathrm{~min}$ \\
\hline $14 \mathrm{~L}$ & $3.5 \mathrm{~min}$ & $4.5 \mathrm{~min}$ \\
\hline $18 \mathrm{~L}$ & $4 \mathrm{~min}$ & $5 \mathrm{~min}$ \\
\hline $20 \mathrm{~L}$ & $5 \mathrm{~min}$ & $6 \mathrm{~min}$ \\
\hline $40 \mathrm{~L}$ & $6 \mathrm{~min}$ & $8 \mathrm{~min}$ \\
\hline $60 \mathrm{~L}$ & $8 \mathrm{~min}$ & $10 \mathrm{~min}$ \\
\hline $80 \mathrm{~L}$ & $10 \mathrm{~min}$ & $12 \mathrm{~min}$ \\
\hline $120 \mathrm{~L}$ & $15 \mathrm{~min}$ & $18 \mathrm{~min}$ \\
\hline $160 \mathrm{~L}$ & $20 \mathrm{~min}$ & $25 \mathrm{~min}$ \\
\hline
\end{tabular}

Table 2.1: Times allowed for air-hydrogen diffusion for various physical volumes at depths of 5 and $10 \mathrm{~m}$.

stick coils are mounted on the middle tier of the CSS tower and are powered by the capacitor bank on the top tier, comprised of nine 9,000 $\mu \mathrm{F}, 40$ Volt DC Mallory capacitors. The discharge of the spark plugs is controlled by LabVIEW software, which allows users to discharge any combination of the nine sparks plugs. A schematic of the ignition system is given in Fig. 2.6.

After the CSS is fired, the spark plugs become wet and do not spark. The nine points of air delivery are oriented in a manner to dry the spark plugs while injecting air into the combustion chamber. The spark plug configuration and points of air delivery are shown in Fig. 2.5. In order to obtain the needed flow rate of air to dry the plugs, the LabVIEW software initiates air flow through the mass flow controller with the solenoid valve closed, which causes the pressure in the hose between the mass flow controller and the solenoid valve to increase. The solenoid valve is then opened a few seconds after the hose becomes pressurized. The opening of the solenoid valve in conjunction with the high pressure in the hose causes a sudden burst of air to pass over the tip of the spark plugs, thus drying them for operation. The spark is then ready to be initiated by the user through LabView software. 


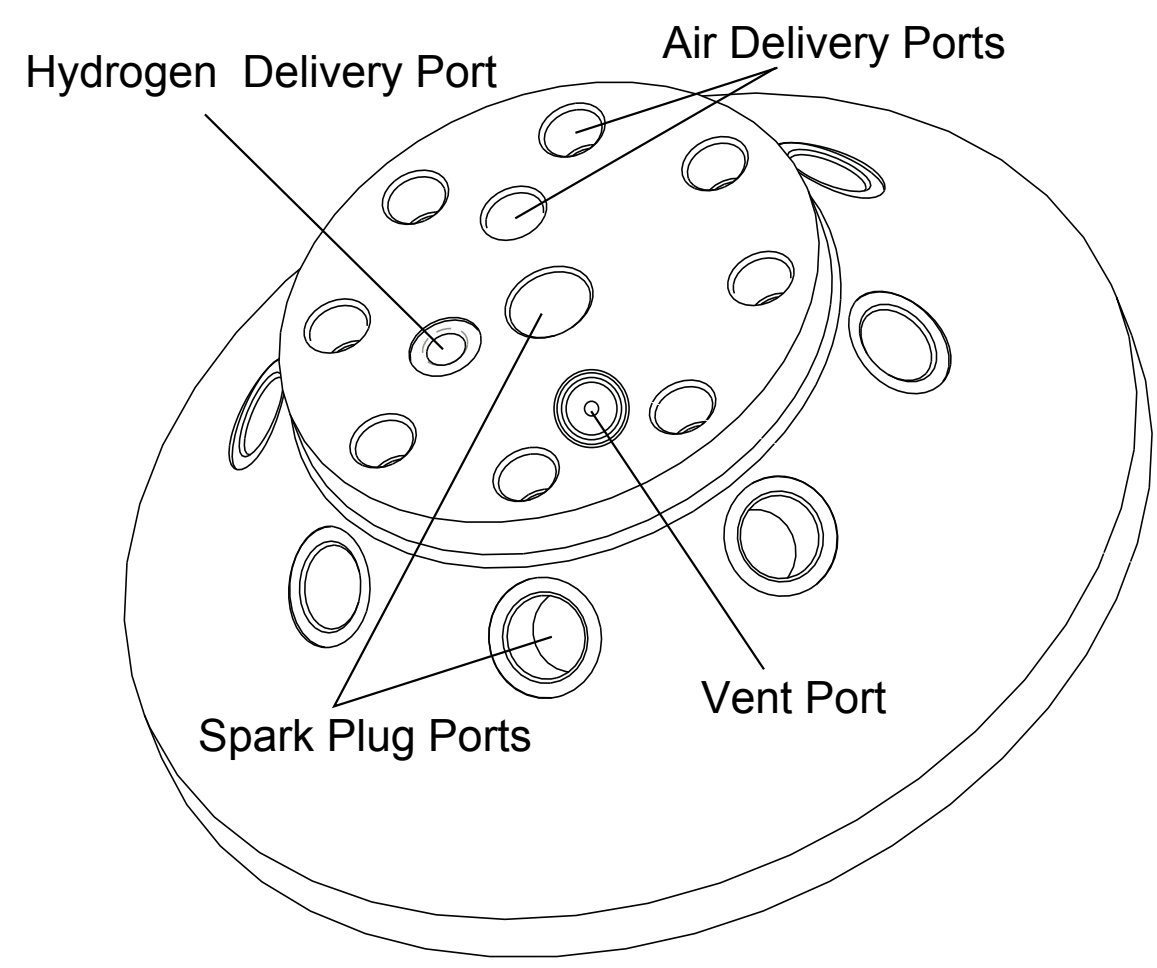

Figure 2.5: The combustion chamber head is shown, containing ports for gas delivery, spark plugs, and vent valve.

\subsection{Additional System Components}

\subsubsection{Power}

Power is provided to the system by an Agilent ${ }^{6}+/-25 \mathrm{~V}, 1 \mathrm{~A}$ DC power supply and an Agilent $40 \mathrm{~V}, 19 \mathrm{~A} 760 \mathrm{~W}$ DC power supply. The needed power is transferred to the system through 330' of Marine Acoustics Cable ${ }^{7}$ containing twisted pair cabling. Each independent circuit can be disconnected at the CSS through the use of SEA CON ${ }^{8}$ underwater connectors.

${ }^{6}$ Agilent Technologies, http://www.home.agilent.com ${ }^{7}$ Cortland Cable Co., http://www.cortlandcable.com/

${ }^{8}$ SEA CON Brantner \& Associates, Inc., http://www.seaconbrantner.com/ 


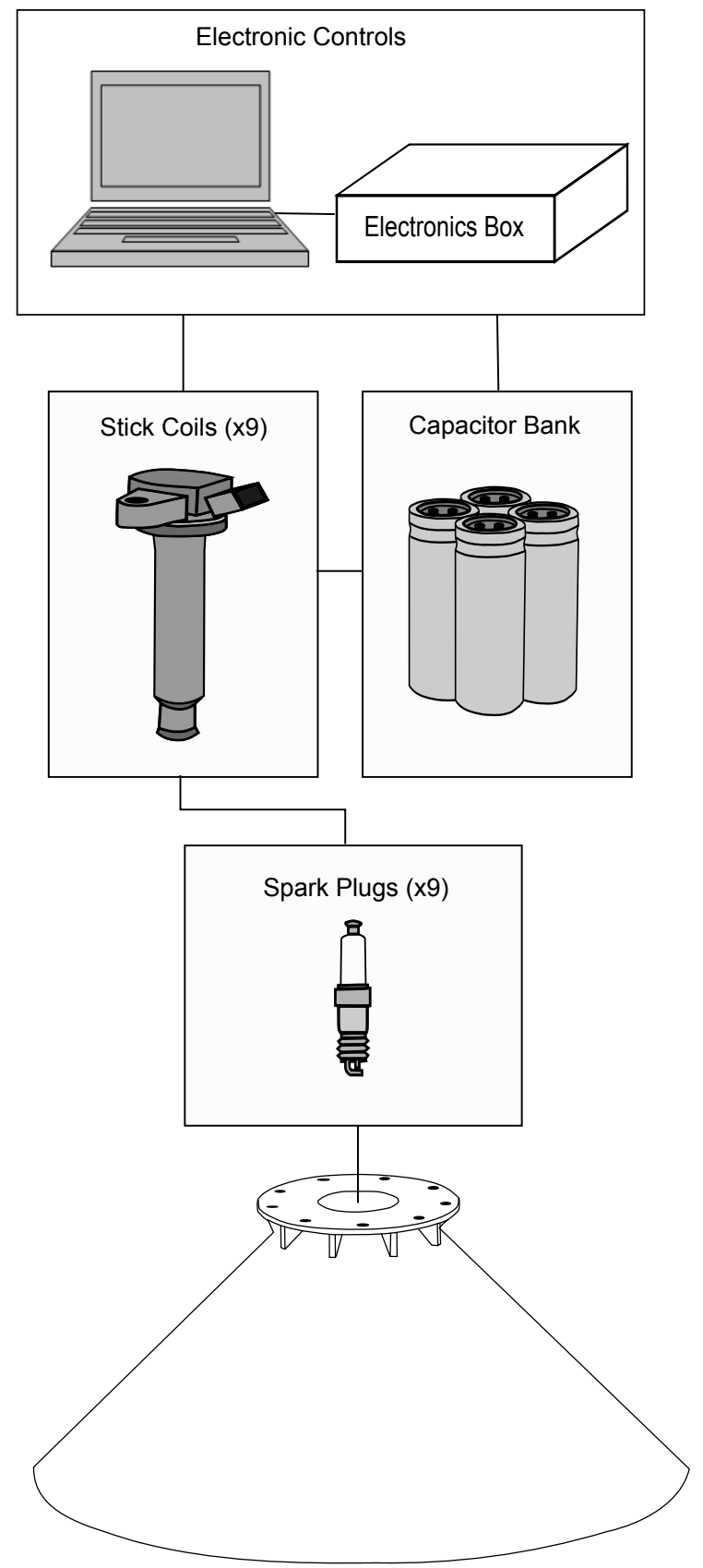

Figure 2.6: A general schematic of the ignition system containing a capacitor bank, stick coils, and spark plugs.

\subsubsection{Vent}

Incomplete combustion and gaseous byproducts of combustion result in a volume 
of gas left in the combustion chamber after firing the CSS. Removing this residual bubble leaves the combustion chamber fully flooded and allows users to accurately fill the chamber with a prescribed volume of fuel and oxidizer. A vent is used to achieve this without surfacing or tipping. The vent process is initiated by opening a solenoid valve connected by tubing to a port in the combustion chamber head. Opening the solenoid valve allows gas to slowly flow out of the combustion chamber, through the tubing, and out of the solenoid valve, and into the water. The user is able to open and close the solenoid valve with LabVIEW controls. If desired, software controls allow the user to prematurely terminate the vent process, leaving a volume of gas in the combustion chamber.

\subsubsection{Water Level Sensor}

To complete the chamber fill routine discussed in Section 3.1, a water level sensor was needed. This sensor is comprised of a probe connected to a circuit powered by a $12 \mathrm{~V}$ signal, as shown in Appendix A. The circuitry was mounted to the apparatus chassis outside of the combustion chamber and connected to the ground of the chassis. The probe was mounted in an opening of the chamber at a slant height $22.5 \mathrm{~cm}$ from the apex of the chamber head, and senses the presence or absence of a conductive path from the tip of the probe to the circuit ground. When no conductive path is available (corresponding to the absence of water) the circuit remains open, but when a conductive path is available (corresponding to the presence of water) the circuit returns a $3 \mathrm{~V}$ signal, which signifies that the probe is wet. 


\subsubsection{Recording System and Electronic Controls}

The recording system consists of two HTI- $90^{9}$ hydrophones cabled to a signal conditioning stage (filtration and preamplification), and a PC-based data acquisition system running LabVIEW software. Acoustic pressure time series were displayed and recorded for each CSS event and spectra were also displayed, for real-time analysis. All aspects of operation and data recording are controlled by a LabVIEW virtual instrument program (VI). A general flow chart of the VI functionality and descriptions of the its subroutines are given in Appendix B. These controls allow the user to vent the combustion chamber, dry the spark plugs, fill the chamber with both gases, ignite the mixture, record the acoustic output, and create a $\log$ file containing the acoustic data and all the operating conditions of the shot. The VI also contains safety features to ensure safe operation of the gas flow control, and to prevent accidental triggering of an event.

${ }^{9}$ High Tech Inc., http://www.hightechincusa.com/ 


\section{Chapter 3}

\section{Description of Experiments}

\subsection{Operation}

Figure 3.1 illustrates a schematic of the experiment. After assembly the CSS and two HTI-90 hydrophones were lowered to the same depth, and an operational routine was followed to fill the CSS with gas and fire on command. The CSS was deployed from a winch, and the hydrophones were deployed from the side of a barge at known distances in the far field. Nylon lifting straps and bungee cords were used as shock absorbers to mechanically isolate the winch and cable from the CSS motion during bubble growth and collapse. Upon ignition the hydrophones recorded the time series waveform of the direct and surface reflected path. After each ignition a predefined operational routine was followed to progress to the next event.

The operational routine was set in place to accurately and repeatably test the CSS for various parameters. Initially the user must set several parameters such as depth of ignition, hydrophone ranges, and combustion chamber size in the LabVIEW software. The combustion chamber is then lowered to the desired depth and any entrapped air is vented, leaving the chamber fully flooded. Next, 


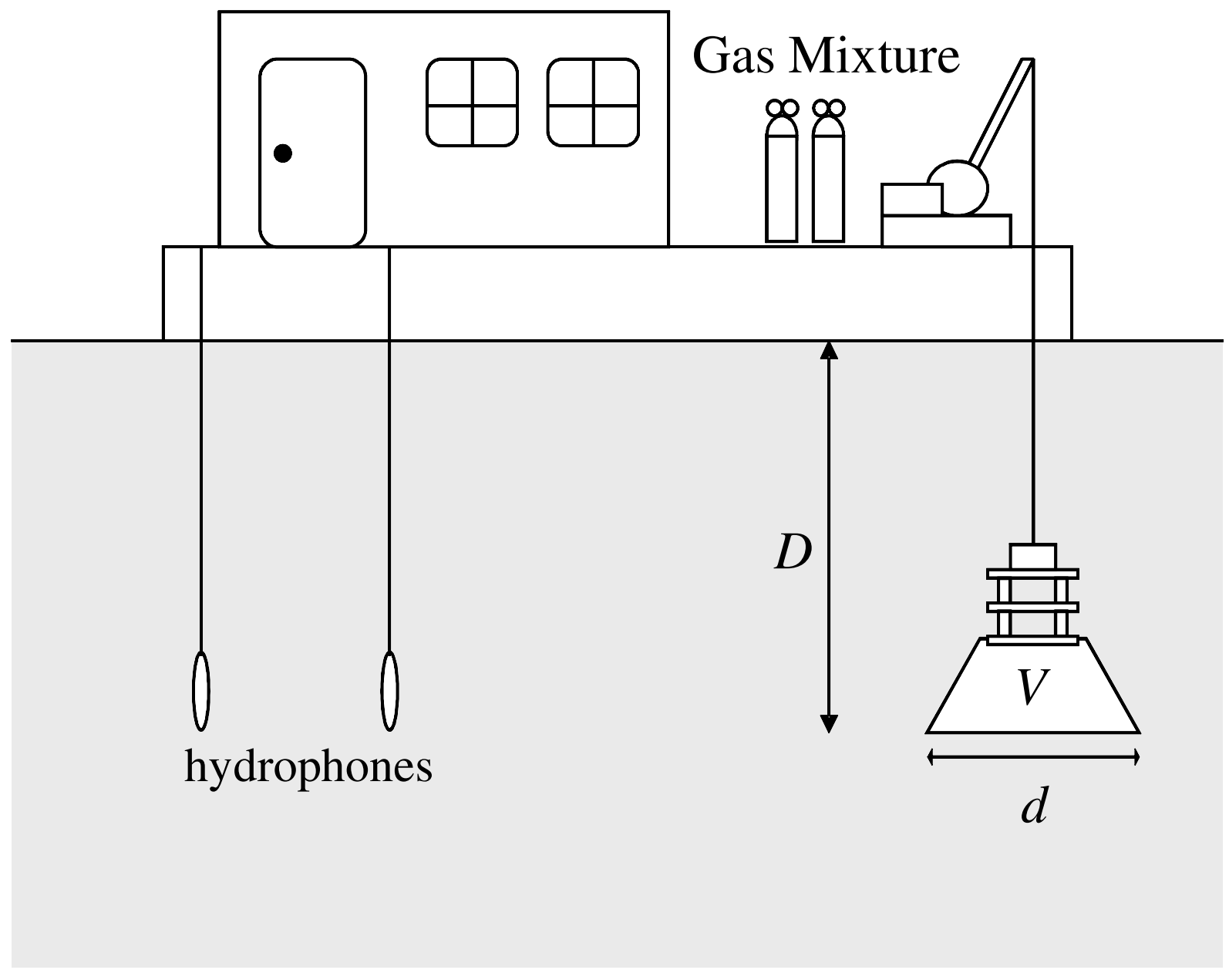

Figure 3.1: A general schematic of the test set up where $D$ is the depth of the CSS and hydrophones, $V$ is the volume of gas in the combustion chamber, and $d$ is the base diameter of the chamber.

the spark plugs are dried with a burst of air resulting in an unknown volume of air in the chamber, as described in Section 2.3. The air must then be vented until the water level sensor indicates wet, leaving 4.2 liters of air in the combustion chamber. The user is now free to add a desired amount of hydrogen or air to the 4.2 liters of air. The combustive mixture must then be given an appropriate amount of time to diffuse after hydrogen is added to the mixture. After diffusion, the user is free to initiate the CSS event. The time series waveform is automatically recorded and 
saved upon firing, and the user is then free to repeat the operational procedure for subsequent events.

Since the gas delivery system was not designed to deliver oxygen, the general operational routine for oxygen-hydrogen events differs from that of the airhydrogen operational routine. Initially the user must set several parameters in the LabVIEW software as in the air-hydrogen operational routine. The combustion chamber is then raised to a depth slightly below the water surface, and the spark plugs are dried with a burst of air. The chamber is then vented so that only a small amount of air remains in the chamber to keep the spark plugs dry. An electrolysis machine is used to generate a desired mass of oxygen and hydrogen, and a tube from the electrolysis machine is held directly below the combustion chamber. Oxygen and hydrogen flow out of the tube and are entrapped below the surface of the water by the combustion chamber. The combustion chamber is then lowered to the desired depth and is ready to be fired. Upon firing, the time series waveform is automatically recorded and saved, and the user is free to repeat if desired.

Since the oxygen-hydrogen mixture is added to the chamber at the surface and lowered to a desired depth, any given mass of gas in a CSS event can be described by two volumetric quantities, the physical volume the gas occupies at depth or the volume the gas occupies at standard temperature and pressure (STP), approximately 300 Kelvin and 1 atmosphere, respectively. For any given mass of gas, the volume occupied at STP will always exceed the physical volume occupied at depth due to the increase in hydrostatic pressure. Therefore, a given mass of gas will be described by the same quantity in terms of volume at STP at any depth, but will be described by a increasingly smaller physical volume for increasing depths. Furthermore, a greater mass of gas is required to produce equivalent 
gas volumes at increasing depths. In this section and the following sections, gas volume will be referred to as volume in liters or volume at STP, corresponding to gas volume at depth and volume at atmospheric conditions. This allows one to compare the affect of depth on the acoustic output for a given volume or given mass at various depths.

\subsection{Description of Experiment}

The experiment was conducted to determine the acoustic output and durability of the new CSS design. Data were collected in a manner to determine the source level and frequency spectrum produced by various combustion chamber sizes, gas volumes, ignition depths, and oxidizing gases. The experiment also allowed for monitoring the durability and robustness of the apparatus throughout testing. Knowledge of both the acoustic output and the hardware robustness are vital in developing a CSS that can repeatedly output the desired acoustic signal without need for repair.

Each data point from the experiment is described by a specific combustion chamber size, volume of gas, depth of ignition, and oxidizing gas. Various combinations of each of these parameters were tested to determine the effect each parameter has on the acoustic output. Repeatability of each data point was tested by consecutively recording three events for each data point before progressing to a subsequent data point. This ensured multiple recordings would be made for each data point of interest and allowed for the smallest volumes to be fired at the beginning of the experiment, and the largest volumes to be fired at the end of the experiment. It was important to test the smallest gas volumes first and slowly increase the gas volume to monitor the stability of the apparatus, and prevent 


\begin{tabular}{|c|c|c|}
\hline & 5 m Ignition Depth & 10 m Ignition Depth \\
\hline \hline 24" Chamber & $6,12,18 \mathrm{~L}$ & $6,10,14,18 \mathrm{~L}$ \\
\hline 36" Chamber & $20,40,60,80 \mathrm{~L}$ & $20,40,60,80, \mathrm{~L}$ \\
\hline 48 " Chamber & $40,80,120,160 \mathrm{~L}$ & \\
\hline
\end{tabular}

Table 3.1: The obtained data points for CSS events using an air-hydrogen mixture.

\begin{tabular}{|c|c|c|c|}
\hline & 5 m Ignition Depth & 10 m Ignition Depth & 18 m Ignition Depth \\
\hline \hline 48 " Chamber & 60 L (STP) & 150 L (STP) & 60 L (STP) \\
\hline
\end{tabular}

Table 3.2: The obtained data points for CSS events using an oxygen-hydrogen mixture.

premature failure. The test matrix for events containing the air-hydrogen mixture is given in Table 3.1, and for events containing the oxygen-hydrogen mixture in Table 3.2.

The experiment began by testing the 24" combustion chamber, with $6 \mathrm{~L}$ of an air-hydrogen mixture at a depth of $5 \mathrm{~m}$. The air-hydrogen mixture is comprised of $70 \%$ air and $30 \%$ hydrogen by volume. Three events were fired with these parameters before continuing to the next data point. Data was then collected with the same combustion chamber, air-hydrogen mixture, and depth for total gas volumes of $12 \mathrm{~L}$ and $18 \mathrm{~L}$. The 24 " chamber was then lowered to a depth of $10 \mathrm{~m}$ and fired with $6 \mathrm{~L}, 10 \mathrm{~L}, 14 \mathrm{~L}$, and $18 \mathrm{~L}$ of the air-hydrogen mixture. Each data point was recorded three times. The 24" chamber was no longer tested after these data points were collected.

The 36" combustion chamber was tested after the $24 "$ chamber. These tests began with $20 \mathrm{~L}$ of the air-hydrogen mixture at a depth of $10 \mathrm{~m}$. The 36 " chamber was then fired with the air-hydrogen mixture at a depth of $10 \mathrm{~m}$ for total gas volumes of $40 \mathrm{~L}, 60 \mathrm{~L}$, and $80 \mathrm{~L}$. After three events were fired at each data point, the 36" chamber was raised to a depth of $5 \mathrm{~m}$. The chamber was then tested with an air-hydrogen mixture for total gas volumes of $20 \mathrm{~L}, 40 \mathrm{~L}, 60 \mathrm{~L}$ and $80 \mathrm{~L}$. 
The 36" chamber was no longer tested after these data points were collected.

Testing continued with the $48^{\prime \prime}$ combustion chamber at a depth of $5 \mathrm{~m}$ with total gas volumes of $40 \mathrm{~L}, 80 \mathrm{~L}, 120 \mathrm{~L}$, and $160 \mathrm{~L}$ of the air-hydrogen mixture. Similarly, a set of three events were fired and recorded for each data point. This concluded the testing of the air-hydrogen mixture.

Experimentation continued with an oxygen-hydrogen mixture comprised of $33.33 \%$ oxygen and $66.66 \%$ hydrogen in the $48 "$ combustion chamber. Three events were recorded at a depth of $5 \mathrm{~m}$ with 60 liters at STP (approx $40 \mathrm{~L}$ at depth). Six events were recorded at a depth of $18 \mathrm{~m}$ with 60 liters at STP (approx. $24 \mathrm{~L}$ at depth). One event was recorded at a depth of $10 \mathrm{~m}$ with 150 liters at STP (approx. $75 \mathrm{~L}$ at depth). This concluded the experiment. 


\section{Chapter 4}

\section{Results}

The following section presents the results of the experiment described in the previous chapter.

\subsection{Effect of Increasing Volume}

As shown in Fig. 4.1, the experiment yielded an overall trend that an increase in gas volume is correlated with an increase in source level (SL). The ESL is shown for events comprised of the air-hydrogen mixture ignited at $5 \mathrm{~m}$ and $10 \mathrm{~m}$ for various volumes (liters at STP) of gas. The trend of increasing SL was expected since an increase in gas volume directly relates to an increase in fuel, which yields an increase in radiated acoustic energy. However, it is seen that the ESL increases logarithmically with respect to an increase in total gas volume. The SL increases at a high rate for small gas volumes, whereas the rate of SL increase is reduced at larger volumes. This implies that the efficiency of energy transfer from potential to acoustic energy is reduced for large volumes of gas. Doubling the volume of gas does not necessarily correspond to doubling the output acoustic energy. It can also be seen that the data points for events at $5 \mathrm{~m}$ and $10 \mathrm{~m}$ follow the same trend and are of comparable magnitude when the volume of gas is calculated in terms of 
volume at STP. This implies that the ESL is depends more on the volume (liters at STP) of gas rather than the depth at which the combustive mixture is fired.

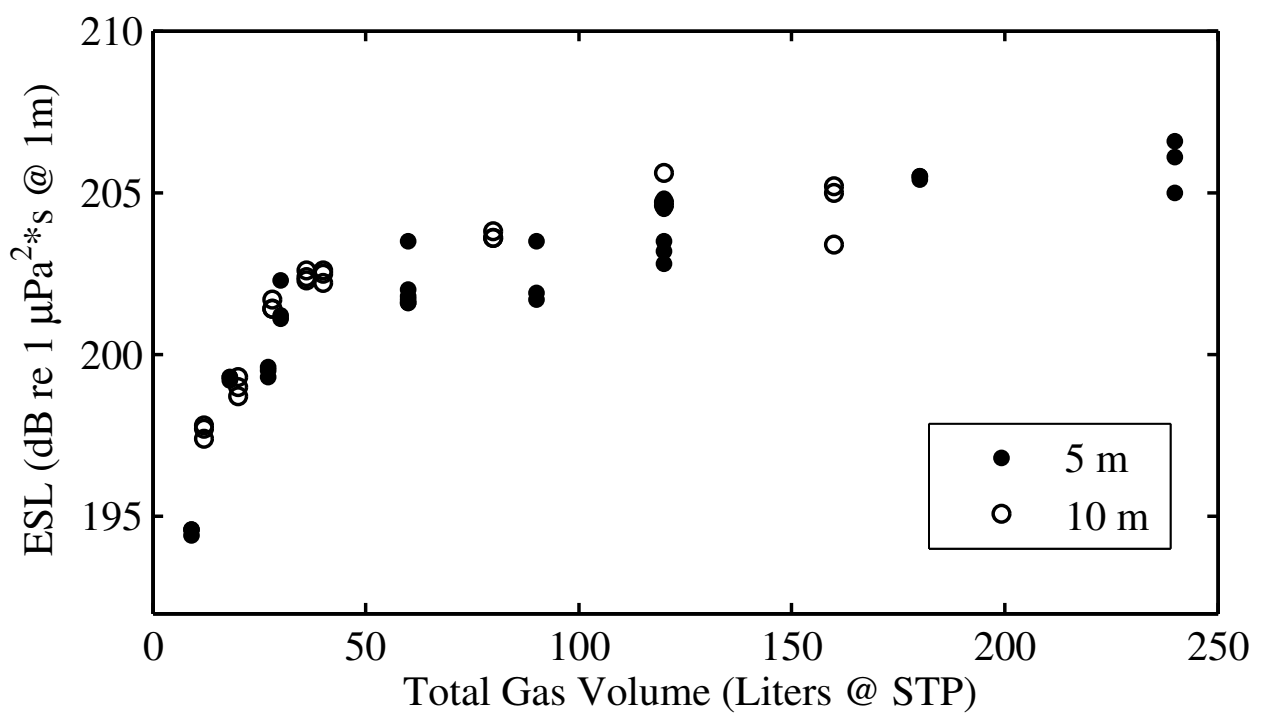

Figure 4.1: Energy Source Levels for total gas volumes at STP of the air-hydrogen mixture at $5 \mathrm{~m}$ and $10 \mathrm{~m}$.

The data also shows that an increase in volume corresponds to a lower fundamental frequency, which agrees with Eq. 1.15. Figure 4.2 shows the fundamental frequency as a function of total gas volume at STP. In this figure the data points for events at $5 \mathrm{~m}$ and $10 \mathrm{~m}$ follow the same trend, but no longer have the same magnitude. This implies that the fundamental frequency is also a function of depth of ignition, which will be discussed in the following section.

\subsection{Effect of Increasing Depth}

Increasing the depth of ignition results in a greater hydrostatic pressure at the bubble-water interface. This allows any given combustion chamber the ability to contain a larger mass of gas at greater depths. In Fig. 4.1 it is seen that 


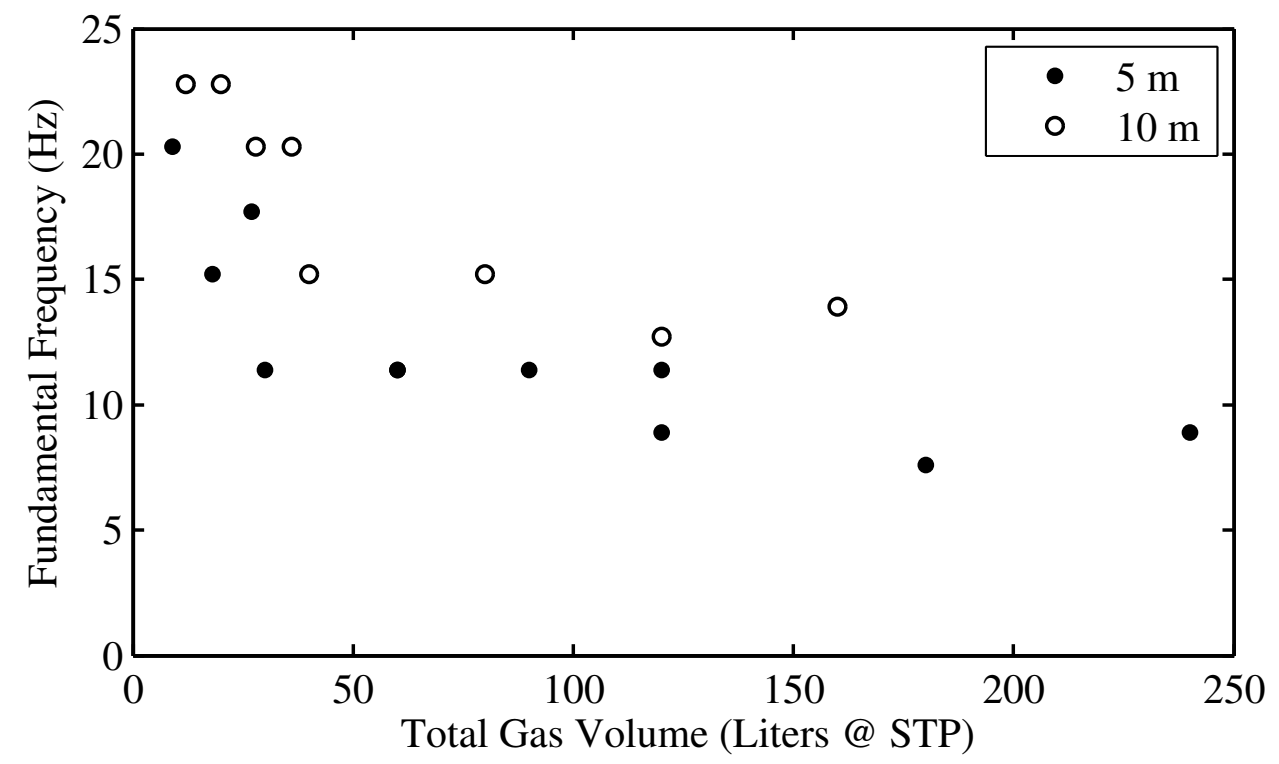

Figure 4.2: Fundamental frequency for total gas volumes at STP of the air-hydrogen mixture at $5 \mathrm{~m}$ and $10 \mathrm{~m}$.

this increase in gas yields a higher SL. Therefore, higher source levels are generally achievable at greater depths, as observed in the experiment described in the previous chapter.

The resultant increase in hydrostatic pressure from increase in depth effects the fundamental frequency as well. Figure 4.2 shows the fundamental frequency for events at $5 \mathrm{~m}$ and $10 \mathrm{~m}$ as a function of volume at STP. It is seen that the fundamental frequency decreases with an increase in gas volume, and increases with ignition depth. This shows that the fundamental frequency is both a function of ignition depth and gas volume, as described in Eq. 1.15.

\subsection{Effect of Oxidizing Gas}

This section analyzes the effect the oxidizing gas has on the acoustic output by comparing events fired with an air-hydrogen mixture to an oxygen-hydrogen mixture. Both mixtures were set to have an equivalence ratio of one, yielding an 
air-hydrogen mixture of $70 \%$ air and $30 \%$ hydrogen by volume, and an oxygenhydrogen mixture of $33.33 \%$ oxygen and $66.66 \%$ hydrogen by volume. For a given total volume of gas mixture, the air-hydrogen mixture has less than half hydrogen content than the oxygen-hydrogen mixture. This section analyzes the SL and energy content within octave bands for $40 \mathrm{~L}$ of each mixture fired in the 48 " chamber at a depth of $5 \mathrm{~m}$.

The differences in the acoustic output are apparent in Figs. 4.3 and 4.4, which show time series data and the ESD for the above mentioned events. Although each event in Fig. 4.3 has a similar fundamental period, the peak pressures experienced by each event and the bubble oscillations after the initial bubble collapse are significantly different. The oxygen-hydrogen mixture is characterized by a sharp peak during bubble collapse, corresponding to the peak pressure of the entire signal. The air-hydrogen mixture is characterized by a much lower pressure during bubble collapse and bubble oscillation for a longer period of time after the initial bubble collapse. Figure 4.4 also shows that the spectral content of an event is affected by the gas chemistry, even if all other metrics (i.e. chamber size, ignition depth, equivalence ratio) remain constant.

The high number peaks and nulls found in the ESD in Fig. 4.4 are partially due to the interference of the direct and surface reflected path. Although the energy contained within spectral bands can be inferred from the ESD, it can be convenient to compare the energy within spectral bands of each event through band-limited ESL calculations. Averaging over bands tends to reduce the effects of fluctuations that result from the nulls, and yields insight into the trends over frequency bands rather than discrete frequencies. Figure 4.5 shows the bandlimited ESL of each event for 11 octave bands centered at 4, 8, 16, 32, 63, 125, 

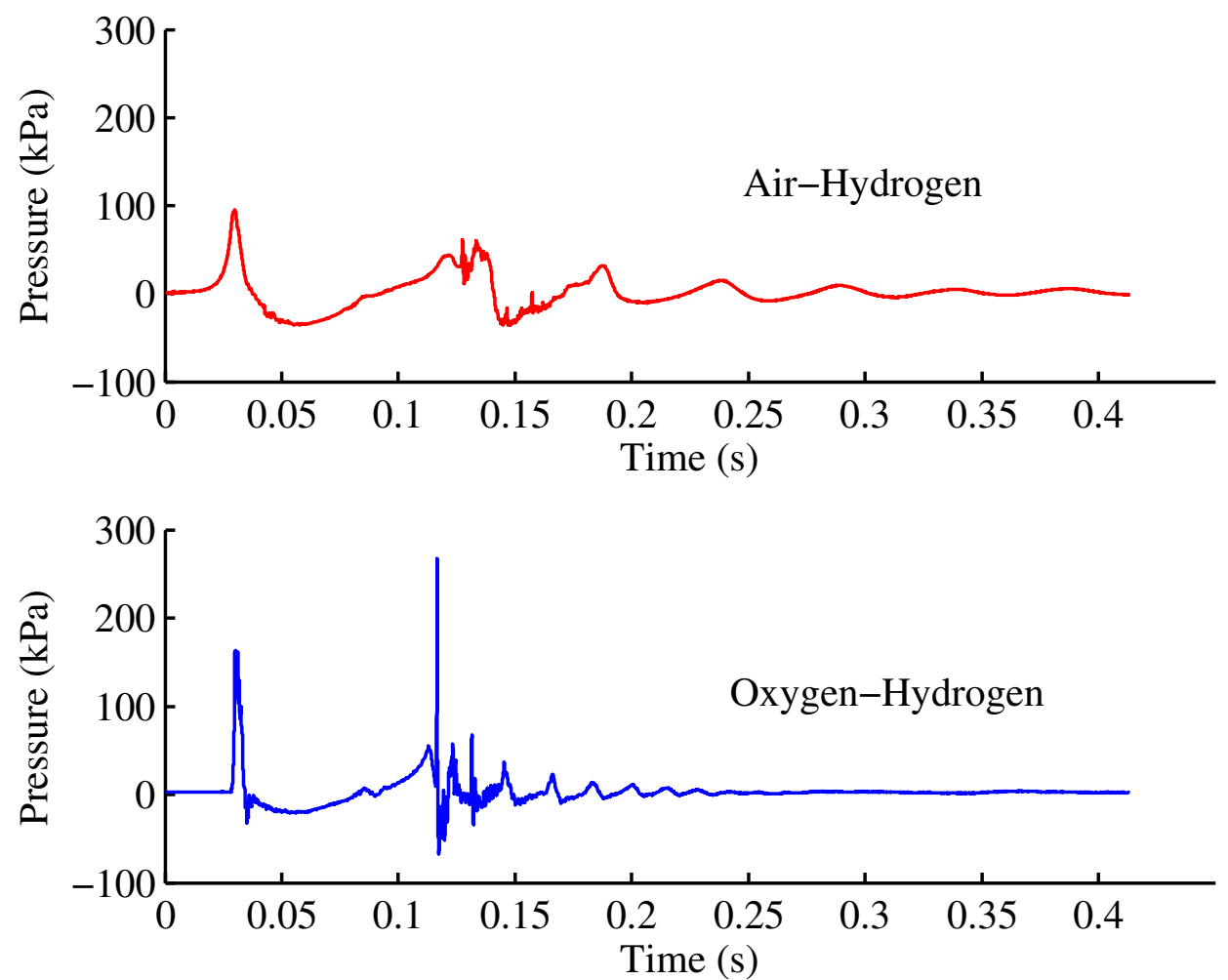

Figure 4.3: Time series data for the air-hydrogen mixture (top) and oxygen-hydrogen mixture (bottom). Both events are comprised of $40 \mathrm{~L}$ of the respective mixture fired at a $5 \mathrm{~m}$ depth in the 48 " combustion chamber.

250, 500, 1000, 2000, and $4000 \mathrm{~Hz}$ respectively.

The total energy contained in the signals are similar with the air-hydrogen event having an ESL of $201.6 \mathrm{~dB}$ and the oxygen-hydrogen event having an ESL of $201.3 \mathrm{~dB}$. However, the energy in the two events is not evenly distributed across each octave band, and the energy within a given band is altered by the oxidizing gas. The air-hydrogen mixture contains the highest amount of energy in the lowest frequency bands, but the amount of energy in the subsequent higher bands tends to decay. The oxygen-hydrogen mixture does not contain as much energy as the air-hydrogen event in the lowest bands, but contains more energy than the air- 


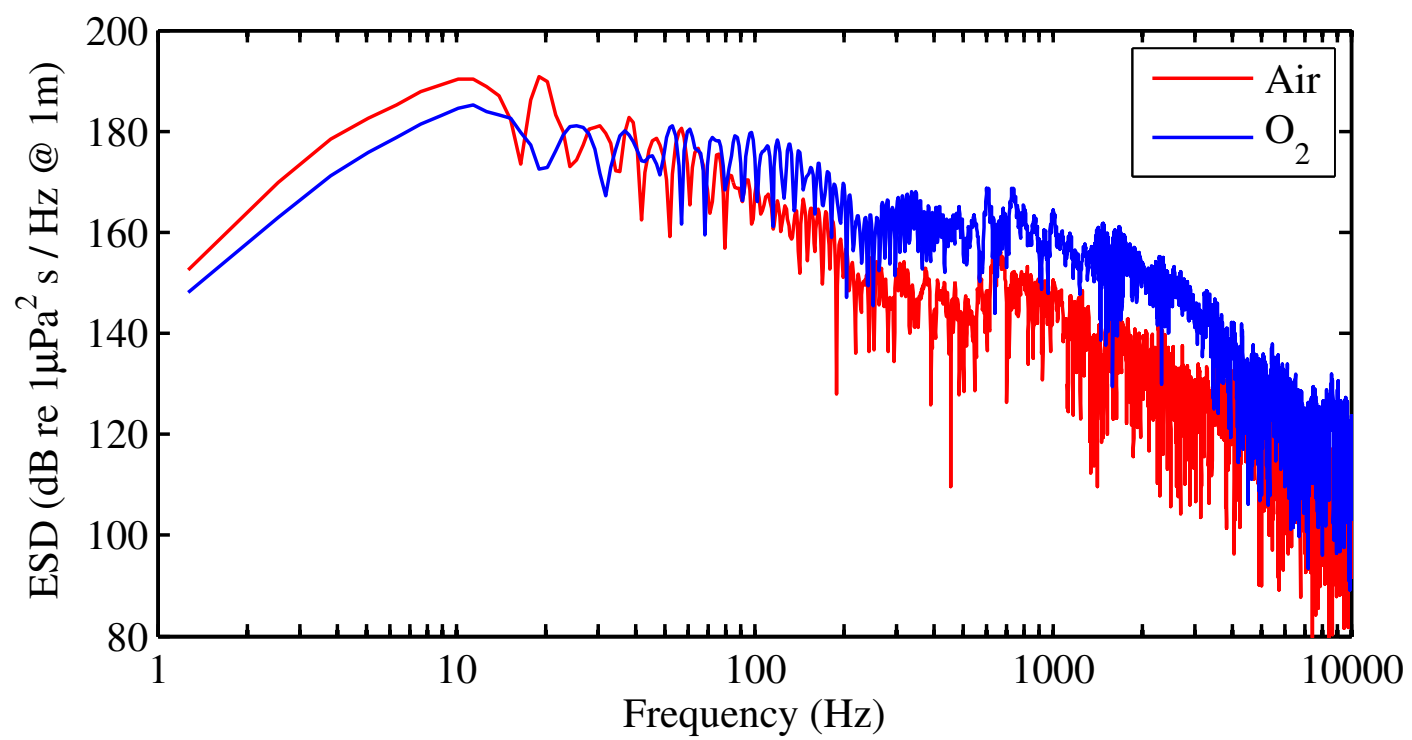

Figure 4.4: Spectra for the air-hydrogen and oxygen-hydrogen mixtures. Both events are comprised of $40 \mathrm{~L}$ of the respective mixture fired at a $5 \mathrm{~m}$ depth in the $48^{\prime \prime}$ combustion chamber.

hydrogen event in the higher bands. From this it is seen that each oxidizing gas produces a different spectral shape.

Source level metrics including peak pressure, SPL, ESL, and ESD used to quantify each event are given in Table 4.1. It is observed that the various metrics used to quantify SL are not always in agreement with regard to which event has the highest SL. For example, in Table 4.1 it is seen that the oxygen-hydrogen mixture has a significantly higher SL when measured as peak pressure. Both events have a relatively equivalent SL when measured as SPL and ESL, but the SL of the air-hydrogen mixture is higher than that of the oxygen-hydrogen mixture when measured as peak ESD. Therefore, one must use caution and explicitly state the metric being used when stating which event has the highest SL. 


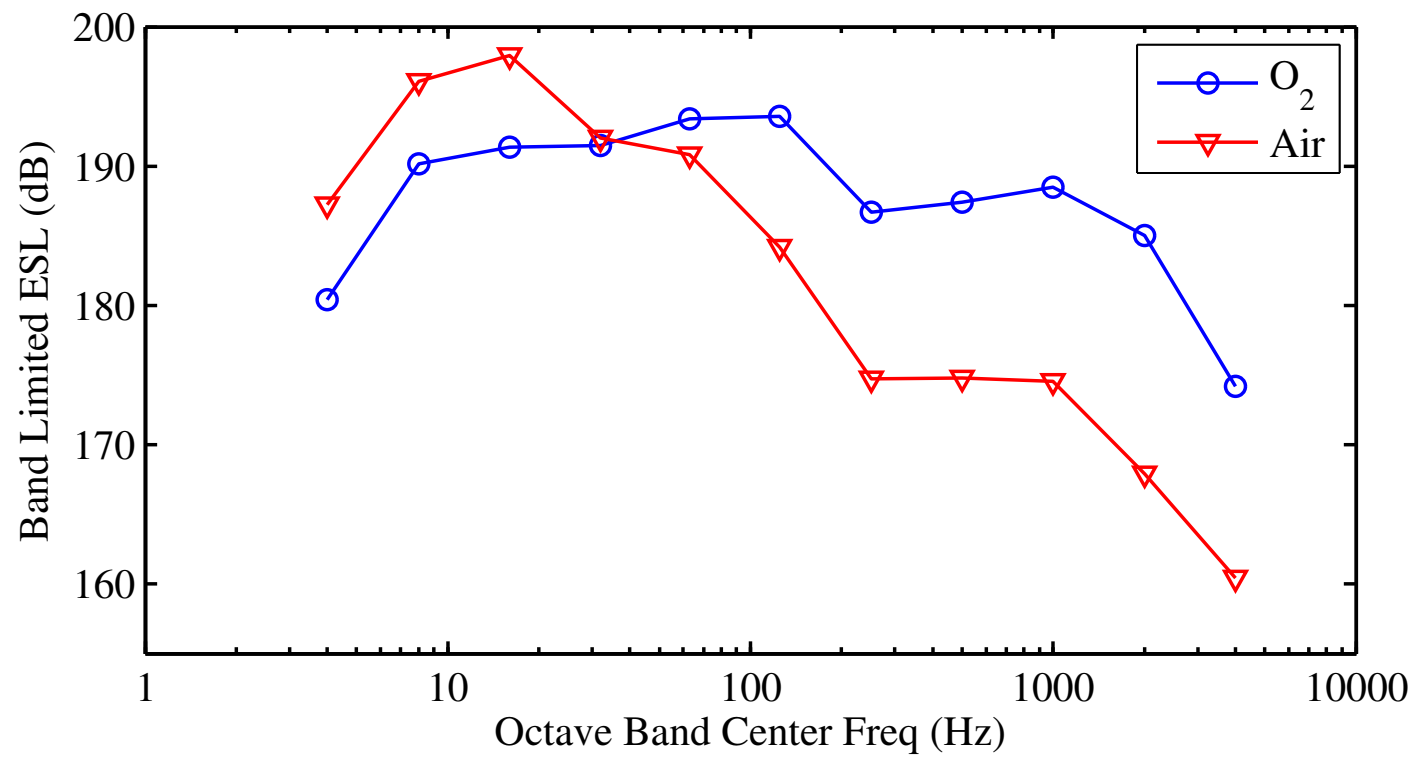

Figure 4.5: Band limited ESL for the air-hydrogen and oxygen-hydrogen mixtures. Both events are comprised of $40 \mathrm{~L}$ of the respective mixture fired at a $5 \mathrm{~m}$ depth in the 48 " combustion chamber.

\begin{tabular}{|c|c|c|}
\hline & Air-Hydrogen & Oxygen-Hydrogen \\
\hline \hline Peak Pressure $(\mathrm{dB})$ & 219.6 & 228.5 \\
\hline SPL $(\mathrm{dB})$ & 205.5 & 206.2 \\
\hline ESL $(\mathrm{dB})$ & 201.6 & 201.3 \\
\hline ESD $(\mathrm{dB})$ & 190.8 & 184.5 \\
\hline
\end{tabular}

Table 4.1: Various source levels for the air-hydrogen and oxygen-hydrogen events. Both events are comprised of $40 \mathrm{~L}$ of the respective mixture fired at a $5 \mathrm{~m}$ depth in the 48 " combustion chamber.

\subsection{Effect of Combustion Chamber Size}

\subsubsection{Constant Percent Fill Volume}

The experiment yielded an expected result of a greater acoustic output with an increase in combustion chamber size for a given percent fill volume. Here the percent fill volume refers to the ratio of the actual gas volume to the chambers theoretical maximum fill volume. As previously shown, the CSS yields an increase in SL for increasing volumes of gas. Therefore, it should be expected that a larger combustion chamber will yield higher SL for a given percent fill volume due to 


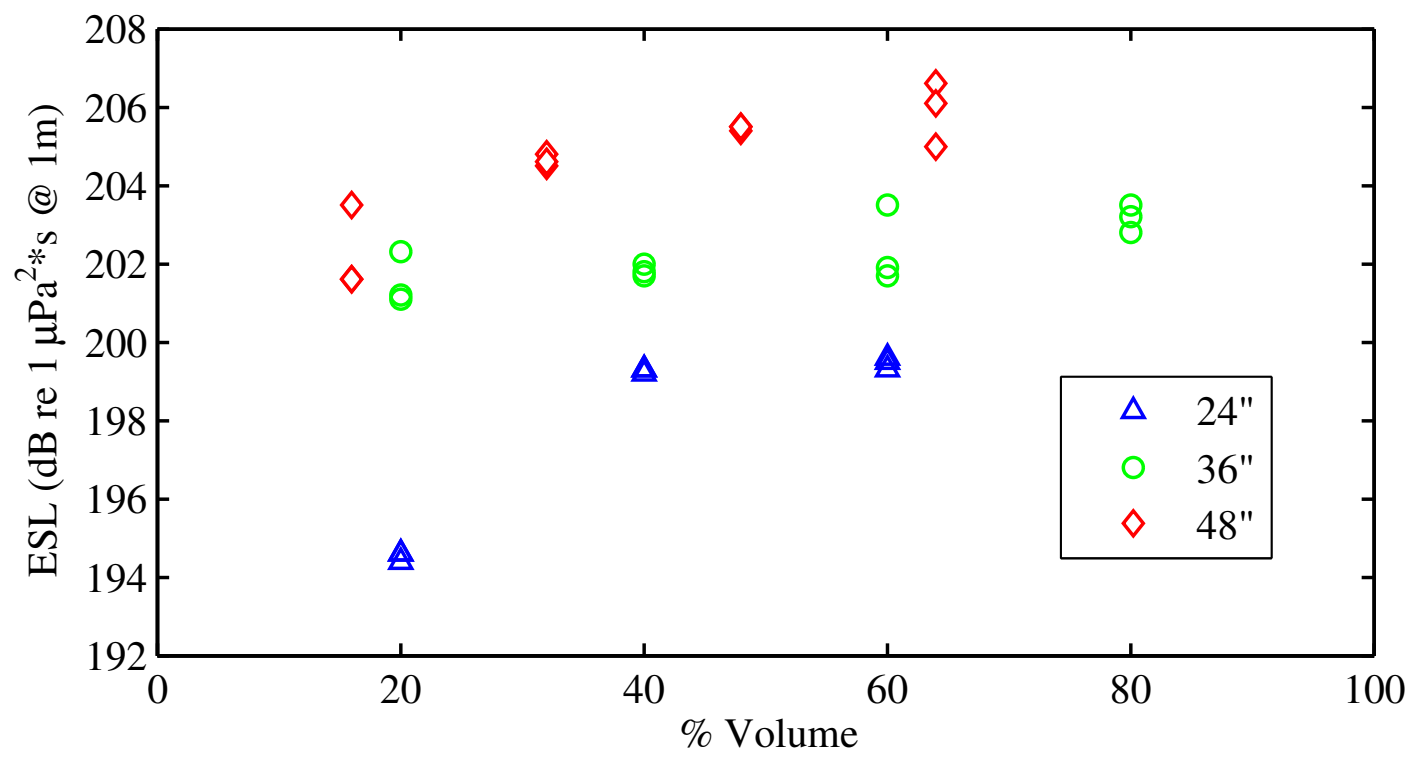

Figure 4.6: ESL for 24", 36", and 48" combustion chambers fired with the air-hydrogen mixture at $5 \mathrm{~m}$ for various percent fill volumes.

the fact that a larger combustion chamber can contain a larger volume of gas.

Figure 4.6 gives ESL as a function of percent fill volume for the 24", 36", and 48 " chambers fired with an air-hydrogen mixture at $5 \mathrm{~m}$ for various percent fill volumes. There is an increase in ESL corresponding to an increase in combustion chamber size for any percent fill volume. It is difficult to give an exact relation for the increase in SL as a function of combustion chamber size, but a rough estimate of 2-3 dB could be assigned as an approximate increase in ESL when increasing the chamber size from the 24 " to 36 " or the 36 " to the 48 " chamber size.

\subsubsection{Bubble Collapse}

Previous CSS experiments [12] have shown the combustion chamber to produce a toroidal shape bubble that expands and subsequently collapses in the chamber. The extent to which the bubble expands beyond the edges of the chamber is determined by the geometry relating the chamber to the contained gas volume. 
An initial gas volume that fills the combustion chamber results in a bubble that expands beyond the edge of the chamber, whereas an initial gas volume that only partially fills the chamber may result in a bubble that never expands beyond the edges of the chamber and is contained within the chamber throughout each oscillation. These geometric differences can result in various gas volumes being expelled from the chamber upon the combustion event. Portions of the initial gas volume may never ultimately collapse within the chamber, but rise to the surface as freely floating bubbles, thus altering the acoustic output.

The collected data supports the hypothesis that differing bubble-to-chamber volume ratios alters the bubble collapse and ultimately the acoustic output. It can be seen that CSS events for which the volume of ignited gas is much smaller than the theoretical volume capacity of the chamber yields a multiple event bubble collapse. It can also be observed that CSS events for which the volume of ignited gas approaches the theoretical volume capacity of the chamber yields a more uniform single-event bubble collapse. One example of this phenomenon can be seen in Fig. 4.7. The top plot in the figure shows time series data from the 36" chamber fired at $10 \mathrm{~m}$ with $20 \mathrm{~L}$ of the air-hydrogen mixture. The bottom plot in the figure shows time series data for an event with all the same parameters except the gas volume was $80 \mathrm{~L}$. Recalling that the 36 " chamber has a theoretical maximum fill volume of $100 \mathrm{~L}$ yields a $20 \%$ fill volume for the top plot an $80 \%$ fill volume for and the bottom plot .

The 20\% fill volume signature does not contain a distinct peak during bubble collapse. The time series data shows a distinct peak, corresponding to the onset of bubble growth, followed by a reduction in acoustic pressure, corresponding to further bubble growth, followed by a short increase in pressure which plateaus 


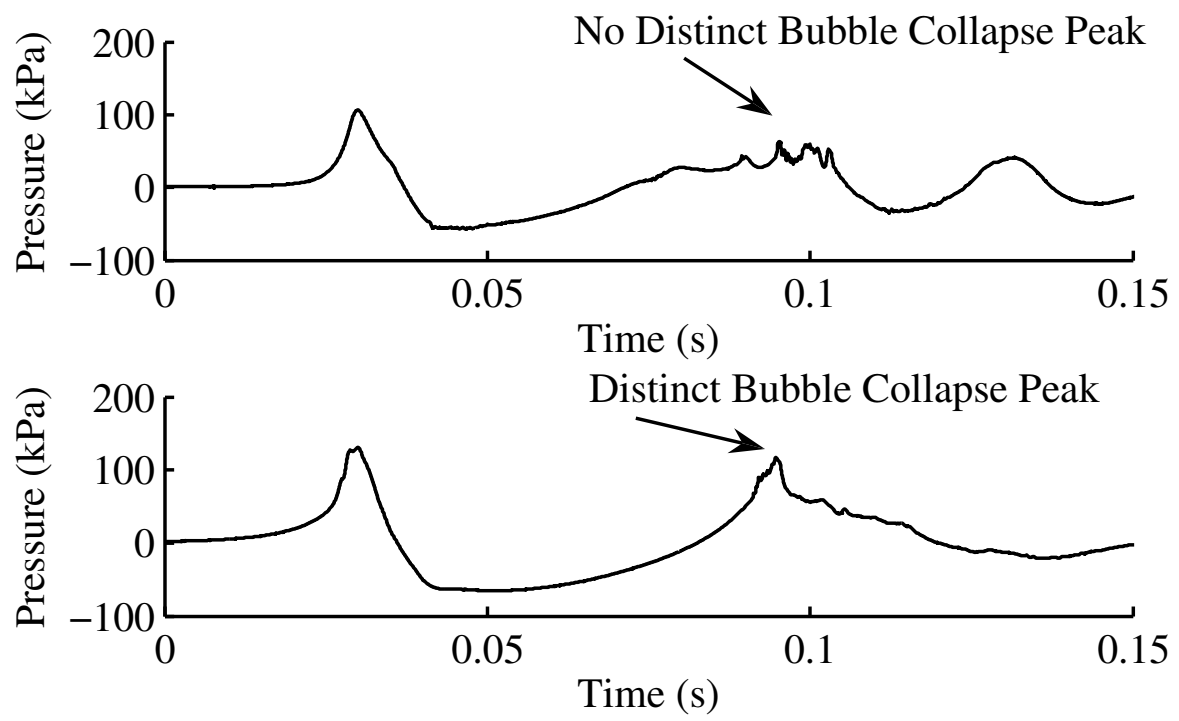

Figure 4.7: Time series data showing bubble collapse features of the air-hydrogen mixture in the $36 "$ combustion chamber fired at a depth of $10 \mathrm{~m}$ for volumes of $20 \mathrm{~L}$ (top) and $80 \mathrm{~L}$ (bottom).

before reaching several local peaks. This is contrasted in the bottom plot, corresponding to $80 \%$ fill volume, containing a distinct peak during bubble collapse. Here the time series data show a similar distinct peak, corresponding to the onset of bubble growth, followed by a reduction in acoustic pressure, corresponding to further bubble growth, follow by a distinct rise in pressure to a sharp point, corresponding to bubble collapse.

This variance in bubble collapse can be seen for each combustion chamber size. Small percent fill volumes yield multiple event collapses while large percent fill volumes yield single event collapses. Predicting the percent fill volume that is required to go from multiple to single event collapses is not yet possible. A more densely populated data set is needed to determine this threshold.

It is hypothesized that low bubble-to-chamber volume ratios result in a bubble that is fully contained with the chamber upon expansion. This results in the toroidal expansion of the bubble where the gas in the center of the chamber sur- 
rounded by the toroidal bubble prematurely collapses before the collapse of the larger toroidal bubble. Since the gas is completely contained within the chamber, all the gas must ultimately collapse into the chamber. Due to the toroidal nature of the bubble, this collapse is not necessarily uniform. Furthermore, it is hypothesized that high bubble-to-chamber volume ratios result in a bubble that expands beyond the chamber edges upon ignition. This results in a similar toroidal expansion, except beyond the edges of the chamber. Since the gas is not completely contained within the chamber, the entire bubble does not necessarily ultimately collapse back into the chamber. In this scenario it is hypothesized that some gas is being expelled from the chamber, which does not collapse but instead rises to the surface, and the gas that does collapse in the chamber does so in a fairly uniform manner. High speed video for various fill volumes could be used to confirm or disprove this hypothesis.

\subsubsection{Constant Volume}

This experiment yielded data for which all parameters were held constant except for the size of combustion chamber. Data were obtained for events composed of the air-hydrogen mixture, ignited at $5 \mathrm{~m}$ for $40 \mathrm{~L}$ and $80 \mathrm{~L}$ in both the $36 "$ and $48 "$ combustion chamber. Figure 4.8 shows the times series data for $40 \mathrm{~L}$ events fired in the 36" and 48" combustion chambers. All parameters are equivalent between these two events except the size of the chamber. Since $40 \mathrm{~L}$ is a relatively small volume of gas in both the 36" and 48" combustion chambers, both times series traces contain the aforementioned plateau effects before bubble collapse. This can be seen between 0.085 and 0.15 seconds on each signature. It should be noted that

the time trace for the event fired in the 48 " combustion chamber has the more 


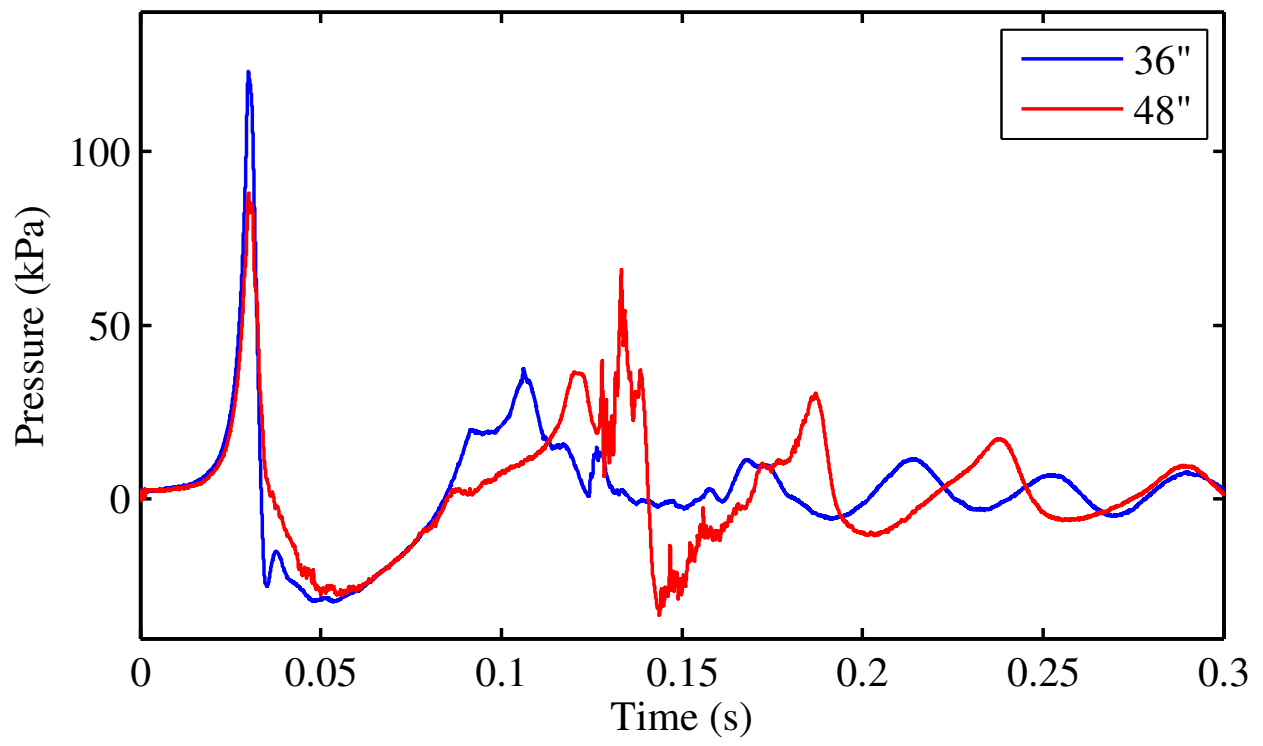

Figure 4.8: Comparison of time series data for $40 \mathrm{~L}$ of the air-hydrogen mixture events fired at a depth of $5 \mathrm{~m}$ in the $36 "$ and 48 " combustion chambers.

distinct multi-phase bubble collapse than the $36 "$ chamber. This could in part be due to the fact that the bubble-to-chamber volume ratio is much lower for the 48 " chamber; hence a multi-phase bubble collapse is predicted as previously discussed.

Although there are apparent differences in the time series plots of these two events, there are only small differences in the output SL of equivalent events in differing chambers. The average SL for peak pressure, SPL, ESL, and ESD are given in Table 4.2 for the $40 \mathrm{~L}$ and $80 \mathrm{~L}$ events using $36 "$ and 48 " chambers. The small data set given in Table 4.2 generally gives a slightly higher SL for the 48" combustion chamber. This could be due to the fact that a larger combustion chamber is able to contain the bubble upon collapse, which can yield a slightly higher acoustic output. Although the presented source levels in Table 4.2 tend to show the 48 " chamber yielding a higher acoustic output, further testing must be done to fully describe the acoustical gain of a larger chamber. 


\begin{tabular}{|c|c|c|c|c|}
\hline & Peak (dB) & $\mathrm{SPL}(\mathrm{dB})$ & ESL (dB) & ESD (dB) \\
\hline 40L, 5m, 36" Chamber & 222.8 & 205.5 & 201.8 & 190.8 \\
\hline 40L, 5m, 48" Chamber & 220.4 & 206.1 & 202.2 & 191.2 \\
\hline $80 \mathrm{~L}, 5 \mathrm{~m}, 36 "$ Chamber & 221.0 & 206.2 & 203.2 & 193.2 \\
\hline $80 \mathrm{~L}, 5 \mathrm{~m}, 48$ " Chamber & 222.5 & 206.8 & 204.6 & 194.2 \\
\hline
\end{tabular}

Table 4.2: Various source level values for the $40 \mathrm{~L}$ and $80 \mathrm{~L}$ of the air-hydrogen mixture fired at a depth of $5 \mathrm{~m}$ in the $36 "$ and $48 "$ combustion chambers.

\subsection{Consistency of Bandwidth}

A unique feature of the CSS is its ability to maintain bandwidth through SL variations. Section 4.1 shows the ability to vary the SL of CSS events, and Section 4.3 shows that each oxidizing gas provides a distinct spectral shape. This section shows the combined effects of these two sections by showing that the spectral shape and bandwidth are maintained as the SL is adjusted for each oxidizing gas.

\subsubsection{Air-Hydrogen Mixture}

Figure 4.9 shows the ESD for two CSS events comprised of the air-hydrogen mixture. The two events shown differ in SL by approximately $10 \mathrm{~dB}$, but it is seen that the spectrum keeps its shape and bandwidth is maintained throughout the change in SL. To further show the relation between the shape of the two events, Fig. 4.10 shows the two curves given in Fig. 4.9 with an amplitude shift to overlay the spectral shapes. Here the curves are plotted on an arbitrary vertical axis in order to more easily compare the spectral shapes regardless of the absolute amplitude of each curve. Figure 4.10 shows that the two events have nearly identical spectral shapes. 


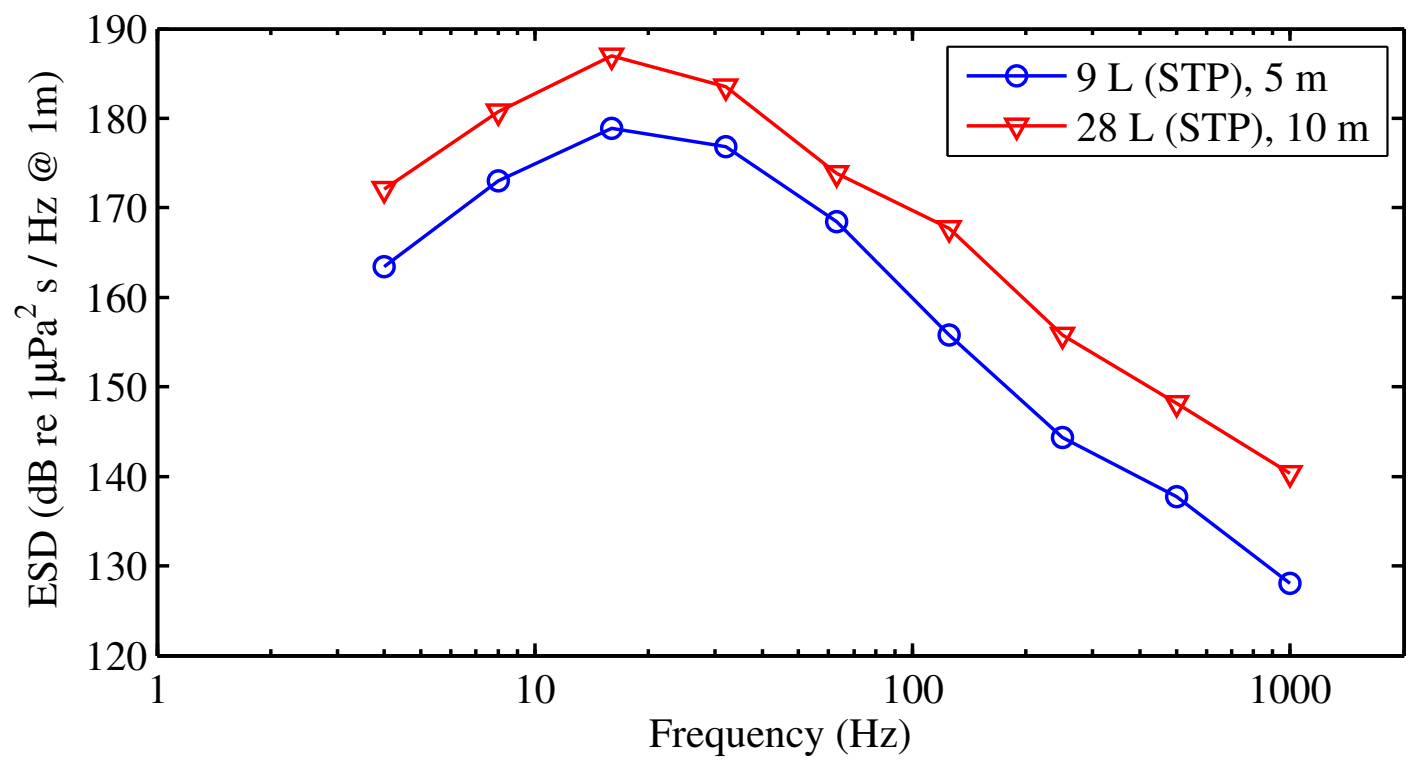

Figure 4.9: ESD of two CSS events comprised of the air-hydrogen mixture. The blue curve is the result of 9 liters (STP) ignited at a depth of 5 meters, and the red curve is the result of 28 liters (STP) ignited at a depth of 10 meters. The ESD is increased by approximately $10 \mathrm{~dB}$ across the spectrum while keeping its shape.

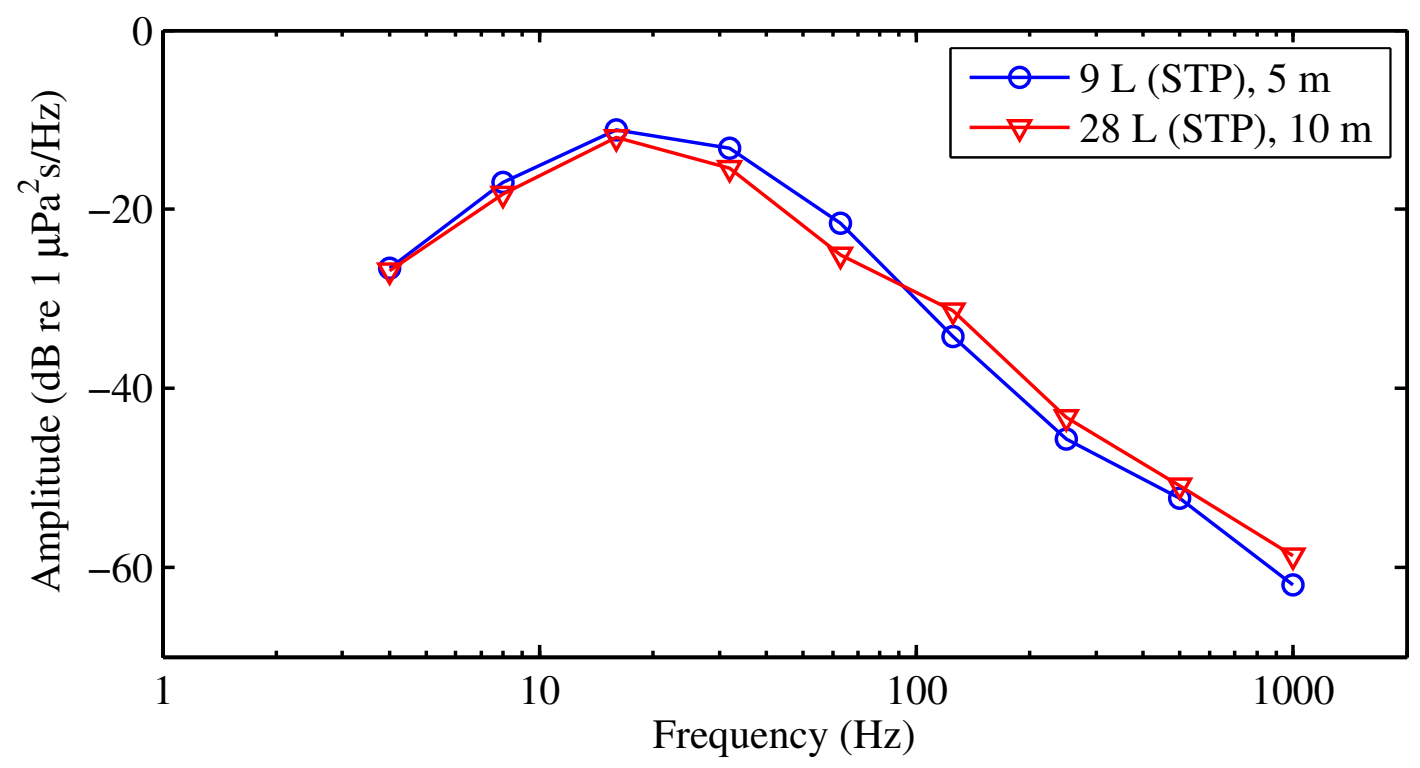

Figure 4.10: Spectral shape of two CSS events shown in Fig. 4.9. The two events are given here on an arbitrary vertical axis to compare the spectral shape without regard to the individual amplitudes of each event. 


\subsubsection{Oxygen-Hydrogen Mixture}

Figure 4.11 shows the ESD for two CSS events comprised of the oxygen-hydrogen mixture. The two events shown differ in SL by approximately $19 \mathrm{~dB}$, but it is seen that the spectrum keeps its same basic shape and bandwidth is maintained throughout SL variations. To further show the relation between the shape of the two events, Fig. 4.12 overlays the spectral shape of the two curves given in Fig. 4.11 on an arbitrary vertical axis in order to compare the spectral shape regardless of the amplitude of each curve. Figure 4.12 shows that the two events have similar spectral shapes with only minor differences. These differences could be due to the fact that the $6 \mathrm{~L}(\mathrm{STP})$ event ignited at $25 \mathrm{~m}$ was recorded during SW06, which was in a different location with a different chamber, than the $150 \mathrm{~L}$ (STP) event ignited at $10 \mathrm{~m}$. The experimental differences could be the cause of the minor differences in spectral shape seen in Fig. 4.12.

\subsubsection{Oxygen-Hydrogen Mixture and Explosive Charge Spectrum Com- parison}

Figure 4.13 shows the ESD for a explosive charge (1.8 lb TNT) detonated at $23.5 \mathrm{~m} \mathrm{[4]}$ and a CSS event comprised of $150 \mathrm{~L}$ (STP) of the oxygen-hydrogen mixture ignited at $10 \mathrm{~m}$. It can be seen that there is a difference in SL of approximately $20 \mathrm{~dB}$, and that the oxygen-hydrogen mixture produces a spectrum similar to that of the explosive charge with reduced amplitude. Figure 4.14 overlays the spectra of the explosive charge and the CSS event on an arbitrary vertical axis to compare the spectral shapes without regard to their absolute amplitudes. When the amplitude of the two spectra are adjusted as shown in Fig. 4.14 it is seen that the spectra show relatively good agreement in spectral shape from $30 \mathrm{~Hz}$ to $500 \mathrm{~Hz}$, with only minor deviations. The differences in the lowest frequency 


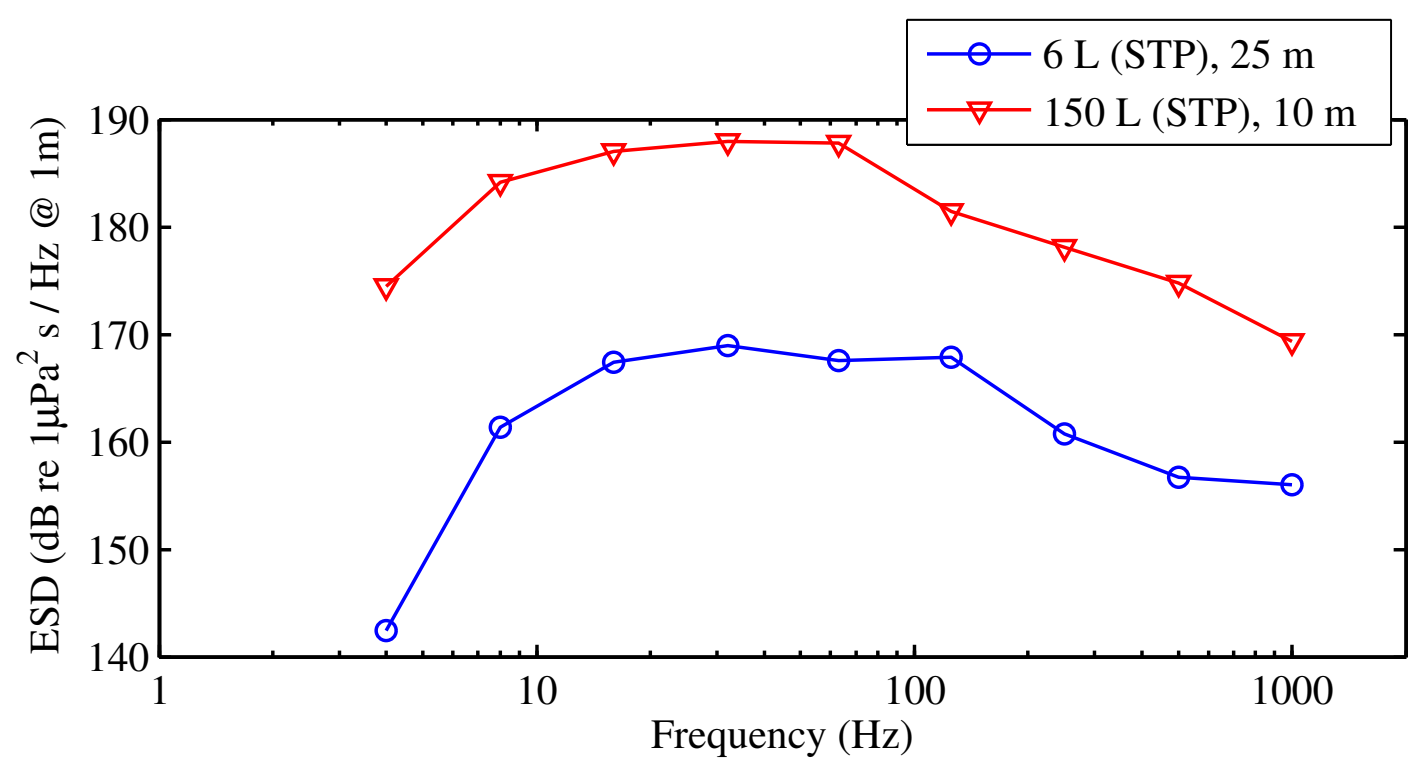

Figure 4.11: ESD of two CSS events comprised of the air-hydrogen mixture. The blue curve is the result of 6 liters (STP) ignited at a depth of 25 meters recorded during SW06, and the red curve is the result of 150 liters (STP) ignited at a depth of 10 meters. The ESD is increased by approximately $19 \mathrm{~dB}$ across the spectrum while keeping its same basic shape.

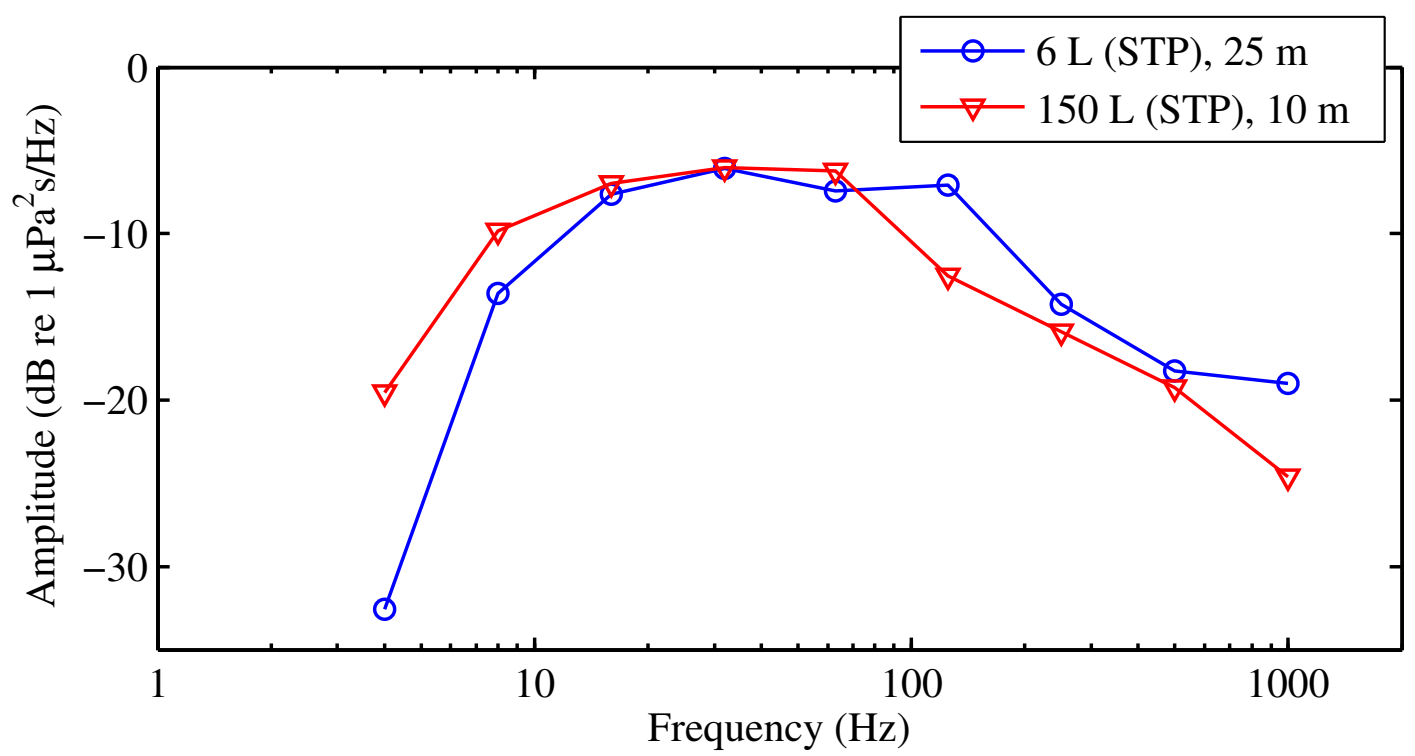

Figure 4.12: Spectral shape of two CSS events shown in Fig. 4.11. The two events are given here on an arbitrary vertical axis to compare the spectral shape without regard to the individual amplitudes of each event. 
bands show that the CSS event does not exactly replicate the spectral shape of an explosive charge; however, it is seen that oxygen-hydrogen CSS events do produce a spectral shape similar to that of an explosive charge and have the advantage of easily being adjusted in SL.

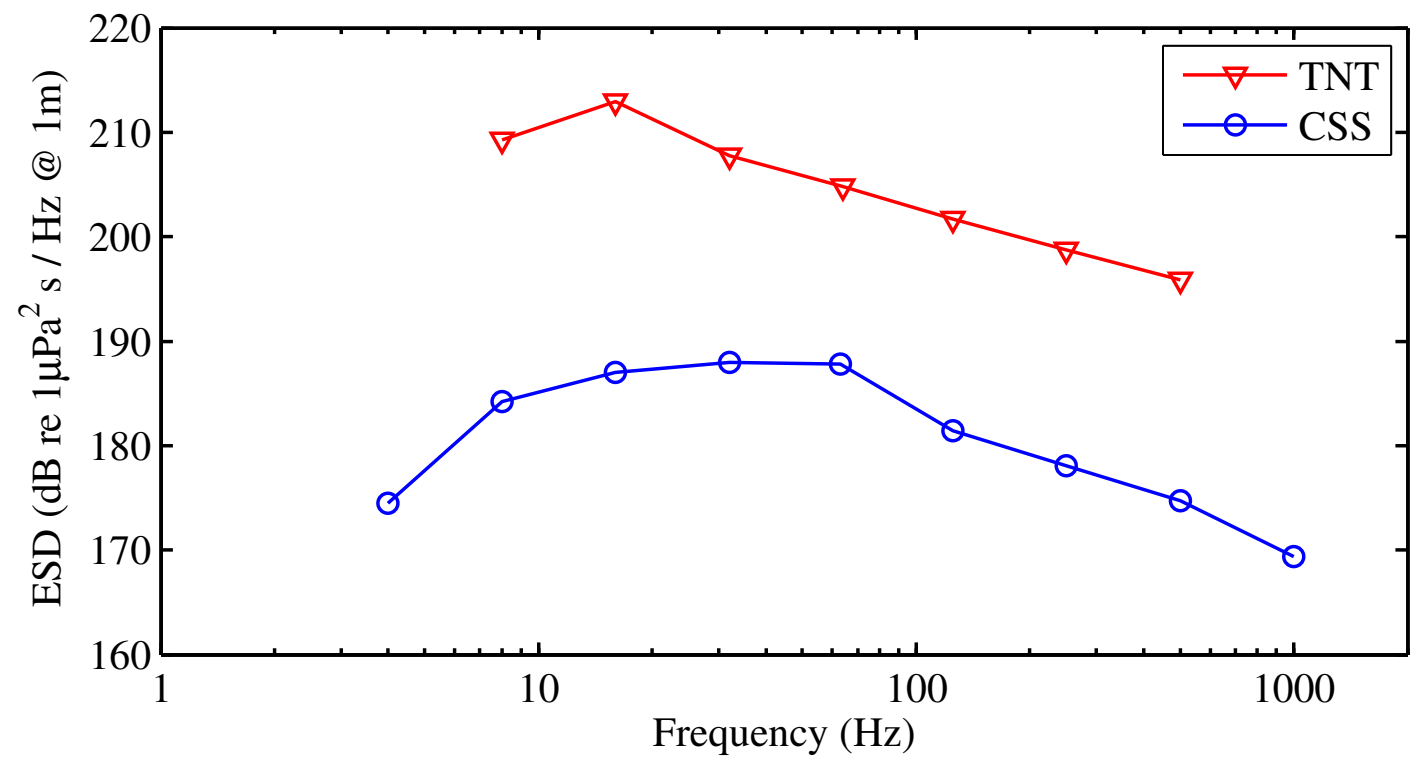

Figure 4.13: ESD of CSS event described by the 150 liters (STP) of the oxygen-hydrogen mixture ignited at 10 meters in the 48" chamber (shown in blue), and ESD of 1.8 lbs of TNT detonated at 23.5 meters [4] (shown in red).

\subsection{Repeatability}

Throughout testing it was seen that the acoustic output of the CSS for any given parameter set can be repeatable, especially at low frequencies. A qualitative assessment of the repeatability can be determined from Appendix C, and a quantitative assessment of the repeatability is given here. This section analyzes the repeatability of the source through peak cross-correlation values in the time domain and cross-spectral analysis in the frequency domain to compare the shape of the signals. The repeatability of the absolute amplitude is obtained by com- 


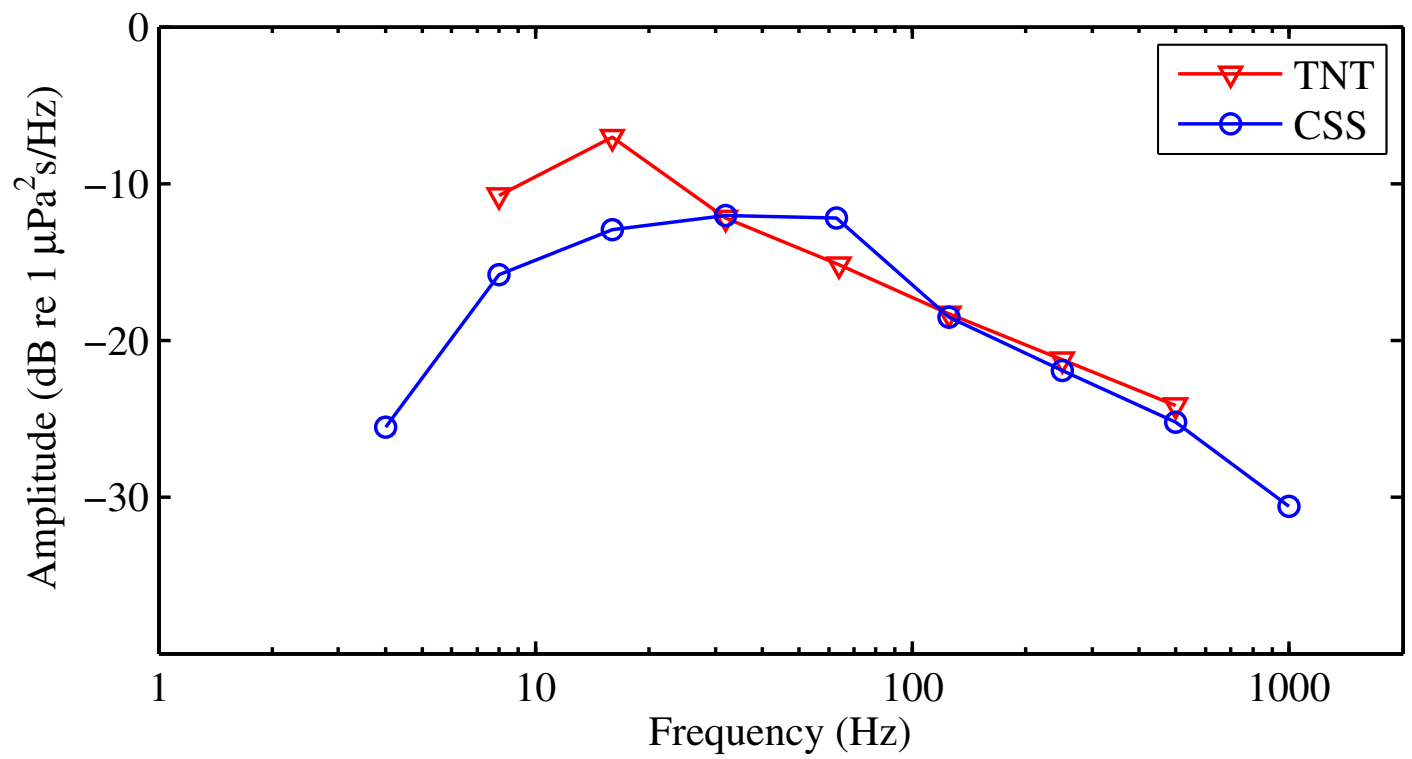

Figure 4.14: Spectral shape of CSS event described by 150 liters (STP) of the oxygen-hydrogen mixture ignited at 10 meters in the 48" chamber (shown in blue), and spectral shape of $1.8 \mathrm{lbs}$ of TNT detonated at 23.5 meters [4] (shown in red). The two events are given here on an arbitrary vertical axis to compare the spectral shape without regard to the individual amplitudes of each event.

paring band limited ESL between similar events. Due to the constraints of the experiment, each parameter set was limited to only three events. This limits the ability to obtain standard deviations for any analysis done within a single parameter set. However, a statistical analysis is performed for frequency bands across all parameter sets.

\subsubsection{Times Series Cross-Correlation}

The peak cross-correlation value was calculated for time series data for every combination of events within a parameter set to quantify the repeatability of the shape of the time series for similar events. This allowed the time series data for each event within each parameter set to be compared with the time series data for every other event in the same parameter set. This method yields three peak cross- 
correlation values for a parameter set containing three events, one value giving the peak correlation of the first event to the second event, one value giving the peak correlation of the first event to the third event, and one value giving the peak correlation of the second event to the third event. The limits of the crosscorrelation are $[0,1]$, where zero corresponds to no correlation and 1 corresponds to perfect correlation.

Table 4.3 shows the maximum and minimum of the three computed peak crosscorrelation values for each parameter set. The maximum value represents the peak cross-correlation value that is calculated for the two events most similar in shape, and the minimum value represents the peak cross-correlation value calculated for the two events most different in shape. It is seen that the CSS is very repeatable for each parameter set containing the air-hydrogen mixture. Each of the highest values for parameter sets containing the air-hydrogen mixture is above 0.96 , and several of these values are 0.99 , which shows the repeatability of events to be near unity.

Events containing the oxygen-hydrogen mixture yield a lower peak cross-correlation value than the air-hydrogen mixture. A value of 0.937 was calculated for the parameter set described by the oxygen-hydrogen mixture in the $48^{\prime \prime}$ chamber, fired at $5 \mathrm{~m}$, and an even lower value of 0.869 was calculated for the parameter set described by the oxygen-hydrogen mixture in the $48^{\prime \prime}$ chamber, fired at $18 \mathrm{~m}$. It was expected that the latter parameter set would have the lowest cross-correlation values due to hardware problems that arose during this portion of testing. However, these lower cross-correlation values are also likely due to the impulsive nature of the oxygen-hydrogen mixture. The sharp peaks associated with this type of gas mixture yields an increase in bandwidth over which a comparison is made, which yields more opportunities for differences in the signal. However, these differences 


\begin{tabular}{|c|c|c|}
\hline Parameter Set & Max Peak C.C. Value & Min Peak C.C. Value \\
\hline $24 ", 5 \mathrm{~m}, 9 \mathrm{~L}$ (STP), Air & 0.997 & 0.994 \\
\hline $24^{\prime \prime}, 5 \mathrm{~m}, 18 \mathrm{~L}(\mathrm{STP})$, Air & 0.999 & 0.997 \\
\hline $24 ", 5 \mathrm{~m}, 27 \mathrm{~L}(\mathrm{STP})$, Air & 0.999 & 0.992 \\
\hline 24", 10m, 12L (STP), Air & 0.998 & 0.909 \\
\hline $24 ", 10 \mathrm{~m}, 20 \mathrm{~L}$ (STP), Air & 0.997 & 0.989 \\
\hline $24 ", 10 \mathrm{~m}, 28 \mathrm{~L}$ (STP), Air & 0.997 & 0.976 \\
\hline $24 ", 10 \mathrm{~m}, 36 \mathrm{~L}$ (STP), Air & 0.998 & 0.988 \\
\hline $36 ", 5 \mathrm{~m}, 30 \mathrm{~L}$ (STP), Air & 0.997 & 0.551 \\
\hline $36^{\prime \prime}, 5 \mathrm{~m}, 60 \mathrm{~L}$ (STP), Air & 0.986 & 0.964 \\
\hline $36 ", 5 \mathrm{~m}, 90 \mathrm{~L}$ (STP), Air & 0.968 & 0.889 \\
\hline $36 ", 5 \mathrm{~m}, 120 \mathrm{~L}$ (STP), Air & 0.992 & 0.978 \\
\hline 36", 10m, 40L (STP), Air & 0.995 & 0.967 \\
\hline $36^{\prime \prime}, 10 \mathrm{~m}, 80 \mathrm{~L}(\mathrm{STP})$, Air & 0.999 & 0.994 \\
\hline $36^{\prime \prime}, 10 \mathrm{~m}, 120 \mathrm{~L}$ (STP), Air & 0.998 & 0.979 \\
\hline $36^{\prime \prime}, 10 \mathrm{~m}, 160 \mathrm{~L}$ (STP), Air & 0.986 & 0.909 \\
\hline $48 ", 5 \mathrm{~m}, 60 \mathrm{~L}(\mathrm{STP})$, Air & 0.994 & 0.924 \\
\hline 48", 5m, 120L (STP), Air & 0.997 & 0.992 \\
\hline 48", 5m, 180L (STP), Air & 0.994 & 0.977 \\
\hline $48 ", 5 \mathrm{~m}, 240 \mathrm{~L}$ (STP), Air & 0.991 & 0.954 \\
\hline $48 ", 5 \mathrm{~m}, 60 \mathrm{~L}(\mathrm{STP})$, Oxygen & 0.937 & 0.900 \\
\hline $48 ", 18 \mathrm{~m}, 60 \mathrm{~L}$ (STP), Oxygen & 0.869 & 0.564 \\
\hline
\end{tabular}

Table 4.3: The maximum and minimum peak cross-correlation values computed for each parameter set. The maximum value represents the peak cross-correlation value calculated for the two events most similar in shape, and the minimum value represents the peak cross-correlation value calculated for the two events most different in shape. The column on the left describes the parameter set by giving the size of the base diameter of the combustion chamber, depth of ignition, total gas volume, and oxidizing gas.

do not necessarily relate to significant differences in the energy found within given bands, as seen in the following section.

\subsubsection{Band Limited Cross-Spectral Analysis}

A band limited cross-spectral analysis was done for each parameter set. Each spectrum was divided into 11 octave bands centered at 4, 8, 16, 32, 63, 125, 250, 500, 1000, 2000, and $4000 \mathrm{~Hz}$. This allowed for specific regions of the spectrum to be analyzed, and yields insight into the repeatability of the CSS in various frequency bands.

The band limited cross-spectra analysis was obtained as follows. Each spectrum 
was divided into the 11 aforementioned octave bands. The peak cross-correlation value was then calculated in each band for each combination of events within a parameter set, as described in Section 1.4. A parameter set containing three events would thus yield 3 sets of 11 peak cross-correlation values. The 3 sets are the result of a comparison between the 1st and 2nd events, the 1st and 3rd events, and the 2 nd and 3rd events. Each of these sets contains 11 peak cross-correlation values corresponding to the 11 octave bands. The highest peak cross-correlation value in each octave band is considered the greatest repeatability obtained during the experiment for each band in a particular parameter set. Table 4.4 gives the highest peak cross-correlation value calculated for each parameter set in the 11 octave bands.

The average band limited peak cross-correlation values from Table 4.4 are displayed in Fig. 4.15 along with the average band limited peak cross-correlation values for the oxygen-hydrogen mixture. Figure 4.15 shows that the CSS is highly repeatable in the lower frequency bands for both the air-hydrogen and oxygenhydrogen mixtures. A peak cross-correlation near unity in the lower frequency bands implies that similar CSS events are virtually indistinguishable with respect to the low frequency content. However, each gas mixture tends to yield lower peak cross-correlation values in the higher frequency bands, especially the air-hydrogen mixture.

Further analysis of Fig. 4.15 shows that the highest peak cross-correlation value for an octave band generally decreases as the center frequency increases. This shows that the current ability to replicate spectra in the lowest frequency bands is possible, but exact replication of the spectra in higher frequency bands is difficult. The difficulty in exact replication of the higher frequency bands could 


\begin{tabular}{|l|c|c|c|c|c|c|}
\hline & $4 \mathrm{~Hz}$ & $8 \mathrm{~Hz}$ & $16 \mathrm{~Hz}$ & $32 \mathrm{~Hz}$ & $63 \mathrm{~Hz}$ & $125 \mathrm{~Hz}$ \\
\hline \hline $24 ", 5 \mathrm{~m}, 9 \mathrm{~L}$ (STP), Air & 1.000 & 1.000 & 1.000 & .999 & .995 & .969 \\
\hline $24 ", 5 \mathrm{~m}, 18 \mathrm{~L}$ (STP), Air & 1.000 & 1.000 & 1.000 & .999 & .999 & .998 \\
\hline $24 ", 5 \mathrm{~m}, 27 \mathrm{~L}$ (STP), Air & 1.000 & 1.000 & 1.000 & 1.000 & .999 & .997 \\
\hline $24 ", 10 \mathrm{~m}, 12 \mathrm{~L}$ (STP), Air & 1.000 & 1.000 & 1.000 & 1.000 & .997 & .983 \\
\hline $24 ", 10 \mathrm{~m}, 20 \mathrm{~L}$ (STP), Air & 1.000 & 1.000 & 1.000 & .998 & .999 & .996 \\
\hline $24 ", 10 \mathrm{~m}, 28 \mathrm{~L}$ (STP), Air & 1.000 & 1.000 & 1.000 & .999 & .993 & .989 \\
\hline $24 ", 10 \mathrm{~m}, 36 \mathrm{~L}$ (STP), Air & 1.000 & 1.000 & 1.000 & .998 & .997 & .996 \\
\hline $36 ", 5 \mathrm{~m}, 30 \mathrm{~L}$ (STP), Air & 1.000 & 1.000 & 1.000 & .999 & .995 & .964 \\
\hline $36 ", 5 \mathrm{~m}, 60 \mathrm{~L}$ (STP), Air & 1.000 & 1.000 & .997 & .963 & .992 & .982 \\
\hline $36 ", 5 \mathrm{~m}, 90 \mathrm{~L}$ (STP), Air & 1.000 & .999 & .968 & .979 & .963 & .863 \\
\hline $36 ", 5 \mathrm{~m}, 120 \mathrm{~L}$ (STP), Air & 1.000 & 1.000 & .998 & .996 & .973 & .925 \\
\hline $36 ", 10 \mathrm{~m}, 40 \mathrm{~L}$ (STP), Air & 1.000 & 1.000 & 1.000 & .995 & .993 & .969 \\
\hline $36 ", 10 \mathrm{~m}, 80 \mathrm{~L}$ (STP), Air & 1.000 & 1.000 & 1.000 & .999 & .999 & .996 \\
\hline $36 ", 10 \mathrm{~m}, 120 \mathrm{~L}$ (STP), Air & 1.000 & 1.000 & 1.000 & .999 & .999 & .968 \\
\hline $36 ", 10 \mathrm{~m}, 160 \mathrm{~L}$ (STP), Air & 1.000 & 1.000 & .994 & .975 & .968 & .876 \\
\hline $48 ", 5 \mathrm{~m}, 60 \mathrm{~L}$ (STP), Air & 1.000 & 1.000 & .999 & .994 & .989 & .941 \\
\hline $48^{\prime \prime}, 5 \mathrm{~m}, 120 \mathrm{~L}$ (STP), Air & 1.000 & 1.000 & .998 & .996 & .994 & .973 \\
\hline $48 ", 5 \mathrm{~m}, 180 \mathrm{~L}$ (STP), Air & 1.000 & 1.000 & .992 & .997 & .989 & .961 \\
\hline $48^{\prime \prime}, 5 \mathrm{~m}, 240 \mathrm{~L}$ (STP), Air & 1.000 & .999 & .987 & .994 & .952 & .856 \\
\hline
\end{tabular}

\begin{tabular}{|c|c|c|c|c|c|}
\hline & $250 \mathrm{~Hz}$ & $500 \mathrm{~Hz}$ & $1 \mathrm{kHz}$ & $2 \mathrm{kHz}$ & $4 \mathrm{kHz}$ \\
\hline $2 \overline{24 "}^{\prime \prime}, 5 \mathrm{~m}, 9 \mathrm{~L}$ (STP), Air & .950 & .916 & .489 & .425 & .137 \\
\hline $24 ", 5 \mathrm{~m}, 18 \mathrm{~L}$ (STP), Air & .982 & .889 & .612 & .359 & .162 \\
\hline $24^{\prime \prime}, 5 \mathrm{~m}, 27 \mathrm{~L}$ (STP), Air & .929 & .571 & .614 & .505 & .169 \\
\hline $24 ", 10 \mathrm{~m}, 12 \mathrm{~L}$ (STP), Air & .948 & .927 & .608 & .452 & .511 \\
\hline $24 ", 10 \mathrm{~m}, 20 \mathrm{~L}$ (STP), Air & .987 & .853 & .704 & .479 & .149 \\
\hline $24 ", 10 \mathrm{~m}, 28 \mathrm{~L}$ (STP), Air & .934 & .827 & .591 & .716 & .482 \\
\hline $24 ", 10 \mathrm{~m}, 36 \mathrm{~L}$ (STP), Air & .988 & .749 & .669 & .488 & .093 \\
\hline $36^{\prime \prime}, 5 \mathrm{~m}, 30 \mathrm{~L}$ (STP), Air & .909 & .825 & .634 & .496 & .428 \\
\hline $36^{\prime \prime}, 5 \mathrm{~m}, 60 \mathrm{~L}$ (STP), Air & .962 & .638 & .549 & .459 & .210 \\
\hline $36^{\prime \prime}, 5 \mathrm{~m}, 90 \mathrm{~L}$ (STP), Air & .713 & .722 & .534 & .603 & .313 \\
\hline $36 ", 5 \mathrm{~m}, 120 \mathrm{~L}$ (STP), Air & .655 & .743 & .688 & .793 & .644 \\
\hline 36", 10m, 40L (STP), Air & .955 & .692 & .570 & .452 & .332 \\
\hline $36 ", 10 \mathrm{~m}, 80 \mathrm{~L}$ (STP), Air & .974 & .604 & .640 & .215 & .134 \\
\hline $36 ", 10 \mathrm{~m}, 120 \mathrm{~L}$ (STP), Air & .895 & .811 & .792 & .561 & .303 \\
\hline $36 ", 10 \mathrm{~m}, 160 \mathrm{~L}$ (STP), Air & .818 & .753 & .489 & .533 & .457 \\
\hline $48^{\prime \prime}, 5 \mathrm{~m}, 60 \mathrm{~L}$ (STP), Air & .815 & .595 & .590 & .339 & .438 \\
\hline $48^{\prime \prime}, 5 \mathrm{~m}, 120 \mathrm{~L}$ (STP), Air & .844 & .377 & .415 & .348 & .510 \\
\hline $48^{\prime \prime}, 5 \mathrm{~m}, 180 \mathrm{~L}$ (STP), Air & .735 & .783 & .771 & .399 & .191 \\
\hline $48^{\prime \prime}, 5 \mathrm{~m}, 240 \mathrm{~L}(\mathrm{STP}), \mathrm{Air}$ & .643 & .748 & .523 & .616 & .284 \\
\hline
\end{tabular}

Table 4.4: The peak cross-correlation values computed for 11 octave bands in each parameter set. This value represents the peak correlation that can found between any two band limited spectra within a parameter set. 


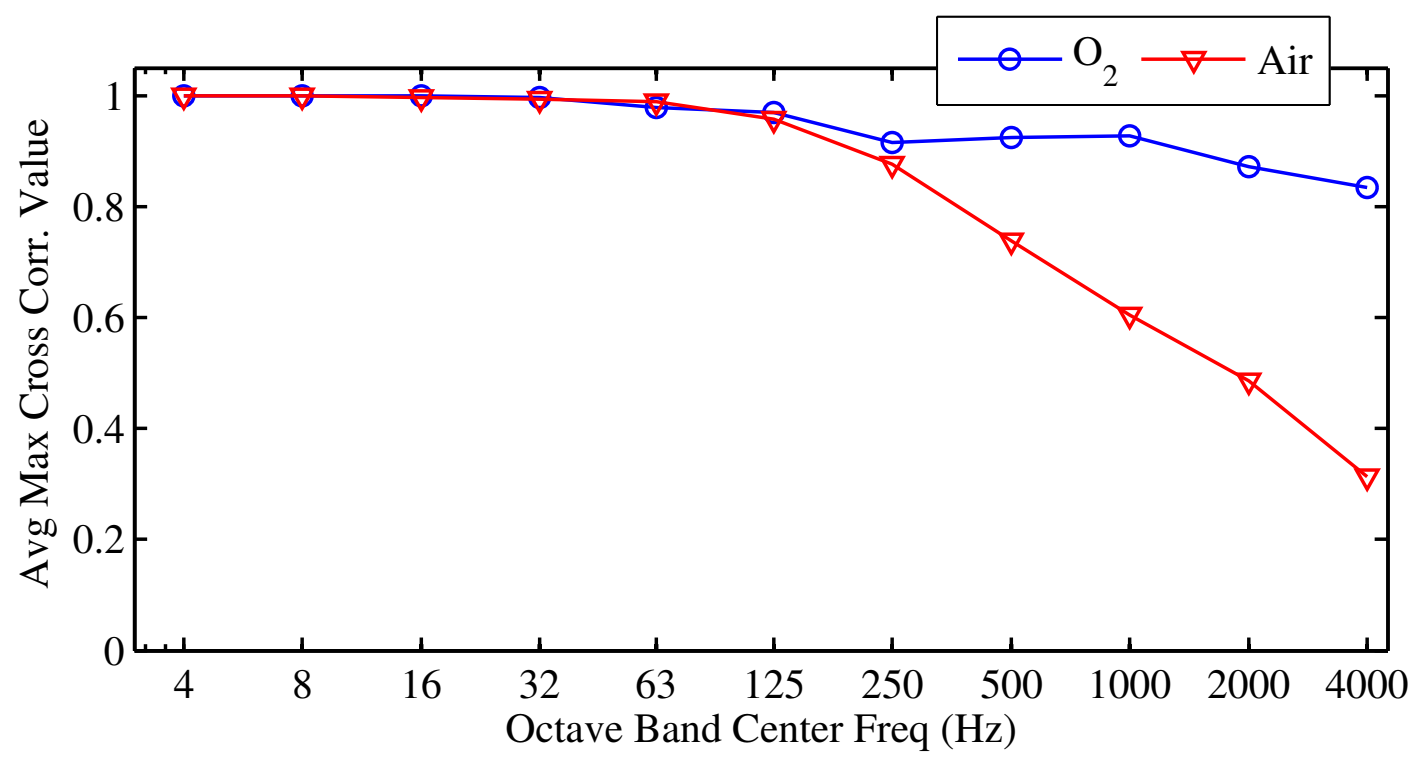

Figure 4.15: The average of the peak cross-correlation values for 11 octave bands for the airhydrogen and oxygen-hydrogen mixtures.

be the result of recording a surface reflection. Each event was fired at a shallow depth, which led to an interference pattern between the direct signal and surface reflection at the hydrophone. It would be very difficult to replicate this interference pattern from event to event, and small phase changes resultant from the surface reflection could greatly lower the peak cross-correlation value calculated in the higher frequency bands. Band limited ESL calculations tend to "smear" out the inconsistencies that arise with small phase changes in spectral plots, and give the total energy contained in a band. This will be analyzed in the following section.

\subsubsection{Band-Limited ESL}

This section analyzes band-limited ESLs calculated for each event across 11 octave bands centered at 4, 8, 16, 32, 63, 125, 250, 500, 1000, 2000, and $4000 \mathrm{~Hz}$. Breaking the spectrum into multiple bands yields insight into the repeatability of the CSS in terms of frequency, as in the band limited cross-spectral analysis. The band 
limited ESL repeatability analysis is similar to the band limited spectra crosscorrelation analysis. Each spectrum was divided into 11 octave bands, and the band limited ESL was calculated for each of the 11 bands for each event for a parameter set. As before, a parameter set containing 3 events yielded 33 band limited ESLs values.

The following analysis was done for each of the eleven bands. Band limited ESL values were calculated for each event within a parameter set. The mean of the band limited ESL values were then calculated for each band. Then the excursion from the corresponding mean value was calculated for each event within a parameter set. For example, a particular parameter set contained three events with band limited ESL values of 197.7, 197.0, and 195.7 dB in a particular band. The mean was then calculated as $196.8 \mathrm{~dB}$, and the respective excursions from this mean were found to be $0.9,0.2$, and $-1.1 \mathrm{~dB}$. This method was used as a means of normalizing band limited ESL values across all parameter sets. A histogram of ESL excursion from the mean for data taken from all the parameter sets containing the airhydrogen mixture is given for each of the 11 bands in Appendix D. The standard deviation of the excursion from the mean across all parameter sets containing the air-hydrogen mixture can then be computed for each octave band and is shown in Fig. 4.16. Figure 4.16 shows that there are generally only small deviations from the mean (less than $1 \mathrm{~dB}$ ) in the lower frequency bands. The higher bands generally yield band limited ESL deviations from the mean ESL of less than $2 \mathrm{~dB}$, with exception of the band centered at $2 \mathrm{kHz}$. 


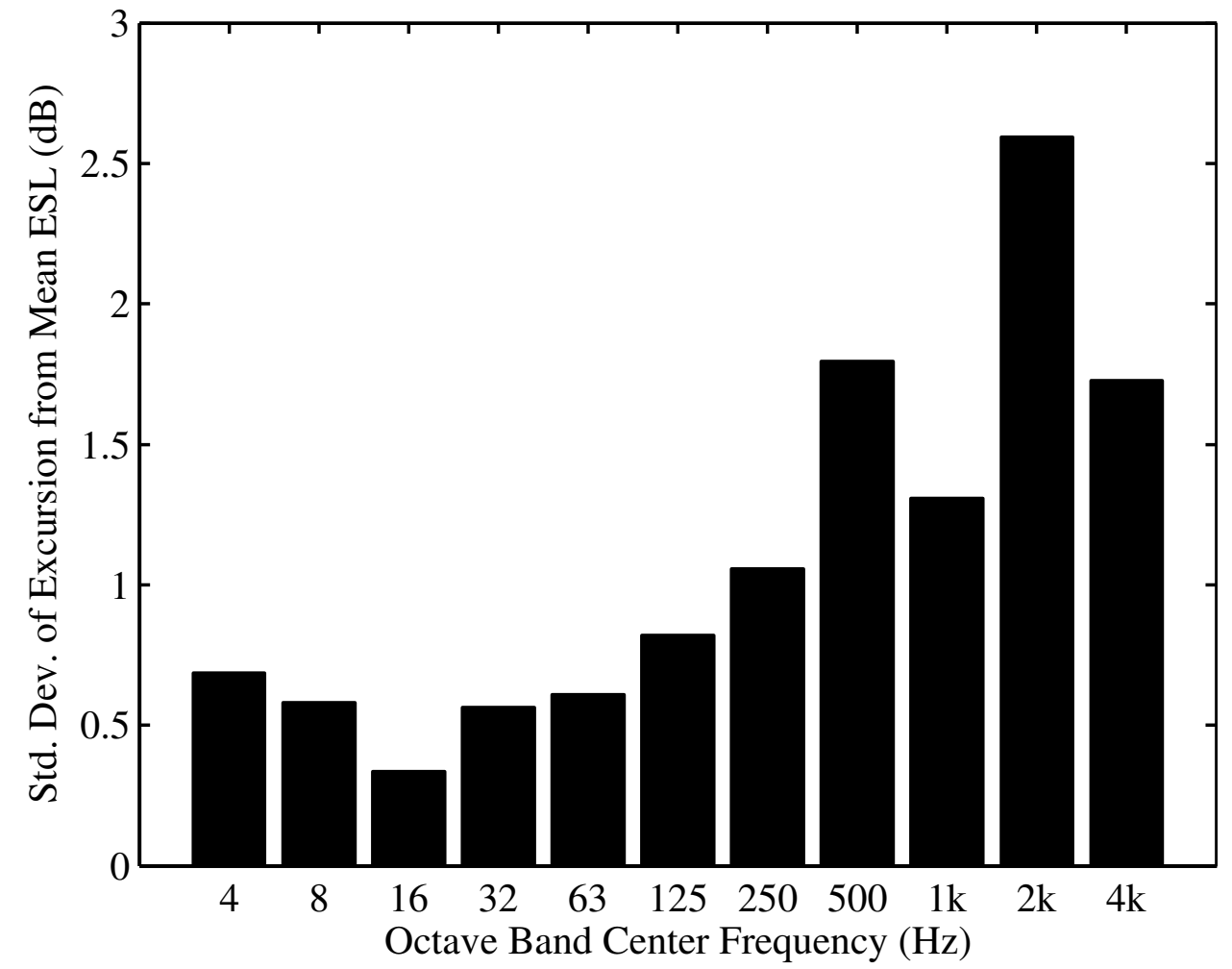

Figure 4.16: Standard deviation of the band limited ESL excursion from the mean across all parameter sets containing the air hydrogen mixture. 


\section{Chapter 5}

\section{Apparatus Robustness}

The goals of the experiment were not only to obtain acoustic data from the CSS but to determine the state of robustness under rigorous testing. In order for the CSS to become a feasible acoustic source it must be able to withstand the rigors of usage over significant periods of time without user maintenance. Ideally, the CSS should be able to operate at a range of depths for hundreds of consecutively fired events with minimum maintenance.

The CSS tested in the August 2009 test required a great deal of user maintenance during testing. The majority of required maintenance throughout testing was due to the repeated impulsive forces experienced by hardware mechanically connected to the apex of the combustion chamber. Hardware including spark plugs, manifold plate, threaded connections, and solenoid valves were all significantly damaged or compromised during testing.

\subsection{Threaded Connections}

The CSS required a great deal of user maintenance to keep all threaded connections securely fit. Users had to continually tighten multiple threaded connections to ensure that the source would not leak and would remain securely bolted to- 
gether upon repeated use. It was seen that even a single event could loosen bolts and screws, putting the apparatus in a temporarily inoperable state. The chamber head that connects the combustion chamber to the gas delivery and ignition systems is an area of major concern. There are several threaded connections covering the chamber head and attached flange which include: bolts connecting the flange to the combustion chamber, bolts connecting the flange to the vertical supports, threaded spark plug connections, and threaded tube connections.

The loosening of the bolts connecting the chamber head flange to the combustion chamber raises two problems. The first problem is that loosening of these bolts relieves the airtight seal between the chamber head and the combustion chamber, which causes leakage. A small gap forms between the combustion chamber and chamber head as these bolts loosen. This allows gas to flow through the top of the combustion chamber, under the chamber head and freely rise to the surface. The second, more extreme, concern is that the bolts will become completely disengaged and allow the combustion chamber to sink to the bottom of the water column. It was witnessed that these bolts can become loose after only a single event. The current method of connecting the combustion chamber to the chamber head must be improved before the desired robustness is reached.

The loosening of spark plugs and threaded tube connections raises similar problems of gas leakage. Gas is allowed to flow out of the combustion chamber and to the surface when the threaded spark plug and tube connections become loose. The loosening of these connections is in part due to their placement at the apex of the combustion chamber. The spark plugs and gas delivery tubes were placed at the apex of the combustion chamber to ignite the gas and blow the spark plugs dry as described in Section 2.2. However, the bubble ultimately collapses to the apex of the chamber transferring the highest amount of pressure to the small area 
around the apex of the chamber. The spark plugs and tube connections receive a sharp high amplitude force, which can loosen their threaded connections in as little as a single event.

Differences were noted in the robustness of the connections around the apex of the chamber when using the air-hydrogen mixture as opposed to the oxygenhydrogen mixture. The combustive process associated with the oxygen-hydrogen mixture leaves little or no gas in the chamber to cushion the impact of bubble collapse. After the oxygen-hydrogen mixture is burned, water violently hammers the apex of the chamber during bubble collapse. Oxygen-hydrogen events were observed to quickly loosen spark plugs and tube connections. However, the combustive process associated with the air-hydrogen mixture results in a gas bubble primarily comprised of nitrogen left unburned in the combustion chamber. Now, after the oxygen contained in air burns with the hydrogen, the water hammering is cushioned by a nitrogen bubble, which protects the connections at the apex of the chamber. The spark plug and tube connections were observed to remain secure for longer periods of time when testing with the air-hydrogen mixture.

Several connections were found to be loose throughout the gas delivery and ignition system connected to the combustion chamber after repeated testing. Generally, threaded connections near the apex of the combustion chamber required the most attention, but threaded connections throughout the apparatus also required attention during testing. Any threaded connection by which a rigid path can be traced to the apex of the combustion chamber will become loose over extensive testing. Therefore, all threaded connections should be removed from a mechanical connection to the apex of the combustion chamber. 


\subsection{Solenoid Valves}

The solenoid valves used in the gas delivery system and vent circuit began to show significant wear and failure throughout the experiment. As described in Section 2.2, each of the two solenoid valves in the gas delivery system, one for air and one for hydrogen, were placed in line with check valves as an attempt to protect them from the shock propagating up from the combustion chamber through the gas delivery tubes and to the solenoid valves. The solenoid valve in the vent circuit was placed in line with stacked stainless steel mesh disks, which attempted to allow passing flow but reduce the edge of a shock wave propagating toward the solenoid valve. However, even with these precautions every solenoid valve experienced problems throughout testing and required replacement.

The Omega SV202 is a normally-closed solenoid valve which requires $12 \mathrm{~V}$ to open. When the $12 \mathrm{~V}$ is applied, current is driven through a coil which magnetically pulls the diaphragm to an open position to allow flow. When $12 \mathrm{~V}$ is not applied the spring returns the diaphragm to the closed position. It was observed that the solenoid valves began to show signs of abuse even after a small number of events fired. The solenoid valves began to show wear as shock waves continually propagated past the check vales and steel mesh into the housing of the valve, which contains the spring and diaphragm. At times the shock wave would cause a misalignment to occur between the diaphragm, spring, and electric coil, which would lock the solenoid in an open or closed position.

It was discovered that slightly increasing the voltage above the recommended $12 \mathrm{~V}$ could snap the diaphragm, spring, and coil back into alignment. This solution of slightly increasing the voltage until alignment would temporarily work before the voltage had to be increased to levels that would potentially damage other pieces 
of hardware. Each solenoid valve ultimately failed and required replacement. It was determined that these solenoid valves were not manufactured to withstand the repeated stresses applied by the shock wave. These valves should not be pneumatically connected with the apex of the combustion chamber. The shock waves propagate through a pneumatic circuit from the apex of the chamber to the solenoid valves and ultimately destroy the valves.

\subsection{Flame Arrestor}

The SGD, Inc. flashback arrestor used to prevent flame propagation into the hydrogen circuit caused several problems throughout testing. The flashback arrestor is a normally open module that allows flow. Sudden increases in pressure or temperature cause the arrestor to automatically close, thus preventing a flame from propagating through hoses containing hydrogen gas. Initially, the flashback arrestor was mounted on the gas delivery manifold. However, it was found that the mechanical shock traveling through the manifold would cause the flashback arrestor to inadvertently close. One must bring the entire CSS apparatus to the surface to manually open the flashback arrestor once it is shut. Although nothing is truly broken, a great deal of time is wasted surfacing the CSS and opening the flashback arrestor before every subsequent event. It was determined that the flashback arrestor should not be rigidly mounted in an area receiving mechanical shock.

It was also determined that the flashback arrestor would not remain in an open position beyond a depth of around $15 \mathrm{~m}$. The arrestor would close as it was lowered to depths exceeding 15 to 20 meters. Similarly, the CSS had to be brought to the surface for users to manually reopen the flashback arrestor. The 
inability of the flashback arrestor to operate below $15 \mathrm{~m}$ greatly limited the depths for which the CSS could repeatedly be fired without surfacing. It was determined that this flashback arrestor should not be used if required to operate beyond a depth of $10 \mathrm{~m}$.

\subsection{Water Level Sensor}

A water level sensor was needed to accurately fill the combustion chamber with a desired volume of gas, as described in Section 2.2. The water level sensor returns a $3 \mathrm{~V}$ signal corresponding to 'wet' when a conducting path is formed between the probe and the circuit ground, and no voltage is returned for an open circuit between the probe and circuit ground, corresponding to 'dry'. Although the mechanical aspects of the water level sensor held up throughout testing, two problematic electrical issues arose during the test. The first common issue with the water level sensor was the pervasive nature of water producing a slight film from the tip of the sensor to the metal threads in the combustion chamber when the chamber is full of gas. This allows a conducting path to be formed between the probe and circuit ground, which incorrectly describes the chamber as full of water instead of gas. Although this issue was eventually solved by covering the probe with a hydrophobic cream, another problem remained. Periodically, a floating ground voltage would arise throughout the manifold. The improper ground reference voltage would cause the water level sensor to incorrectly return a wet/dry signal. The misbehavior of the water level sensor could ultimately shut down testing if not properly working. Therefore, the current design of the water level sensor is not satisfactory. 


\subsection{Spark Plugs}

The spark plugs were not only found to become loose after testing but at times were found to physically break into two separate pieces. This became a time consuming malfunction since the CSS must be brought to the surface to replace the broken spark plugs. The current location of the spark plugs is at the apex of the combustion chamber which is the most violent area of bubble collapse. The spark plugs used in the August 2009 tests were not durable enough to withstand the repeated blast of the CSS. 


\section{Chapter 6}

\section{Conclusions}

This thesis has presented the further development, the current status, and an analysis of a unique high amplitude, low frequency underwater sound source called the Combustive Sound Source. Chapter 1 presented the background and history of the CSS including previous findings and analysis. The purpose of developing a CSS was discussed to give insight into the overall goals of the experiment. Theory and metrics for quantifying impulsive sound sources were presented as a precursor to the analysis.

Chapter 2 provided a description of the experimental apparatus to present the details of the hardware. This section included the description of the combustion chambers, the gas delivery system, the ignition system, and the various hardware features. Insight was given into the overall development of the apparatus and why specific hardware features were added.

Chapter 3.1 then explained the operation of the system and described an experiment (August 2009). Procedures that must be taken to successfully fire the CSS and collect the desired data were described. The details of the experimental procedure were outlined to allow for replication of the experiment if desired.

The results and analysis of the August 2009 experiment were then presented. The analysis used a set of metrics for quantifying impulsive sound sources de- 
scribed in Chapter 2. The analysis of the experimental data included quantifying the effect of increasing the gas volume, the effect of increasing the depth of ignition, the effect of the oxidizing gas, the effect of the combustion chamber size, and the repeatability of the source. The repeatability of the source was analyzed and presented in the time and frequency domains for various events. The repeatability section included analysis of the time series cross-correlation of separate events, band limited cross-spectral analysis, and the repeatability of band limited ESL values for various events.

The analysis showed that an increase in gas volume yields an increase in SL, and that higher source levels are generally achievable at greater depths. It was also shown that the fundamental frequency of an event increases with ignition depth and decreases with gas volume. The ability to produce various time series and spectra with maintained bandwidth was shown for air-hydrogen and oxygenhydrogen gas mixtures. Finally, The repeatability of the source was shown to be high through metrics quantifying the shape and amplitude of various events.

An analysis of the apparatus robustness during the August 2009 experiment was also presented. Insight was given into problematic issues that arose during testing. These issues included: threaded connections, solenoid valves, flame arrestor, water level sensor, and spark plugs. The problems caused by each of these areas were detailed to give insight into future designs of the CSS.

This work presents a continuing effort to fully understand and optimize the Combustive Sound Source. While continued research is required to design a CSS that will be useful for field work in underwater acoustics, this thesis shows the potential for the CSS to become a viable high amplitude, impulsive underwater sound source. 


\section{Appendix A}

\section{Water level sensor}

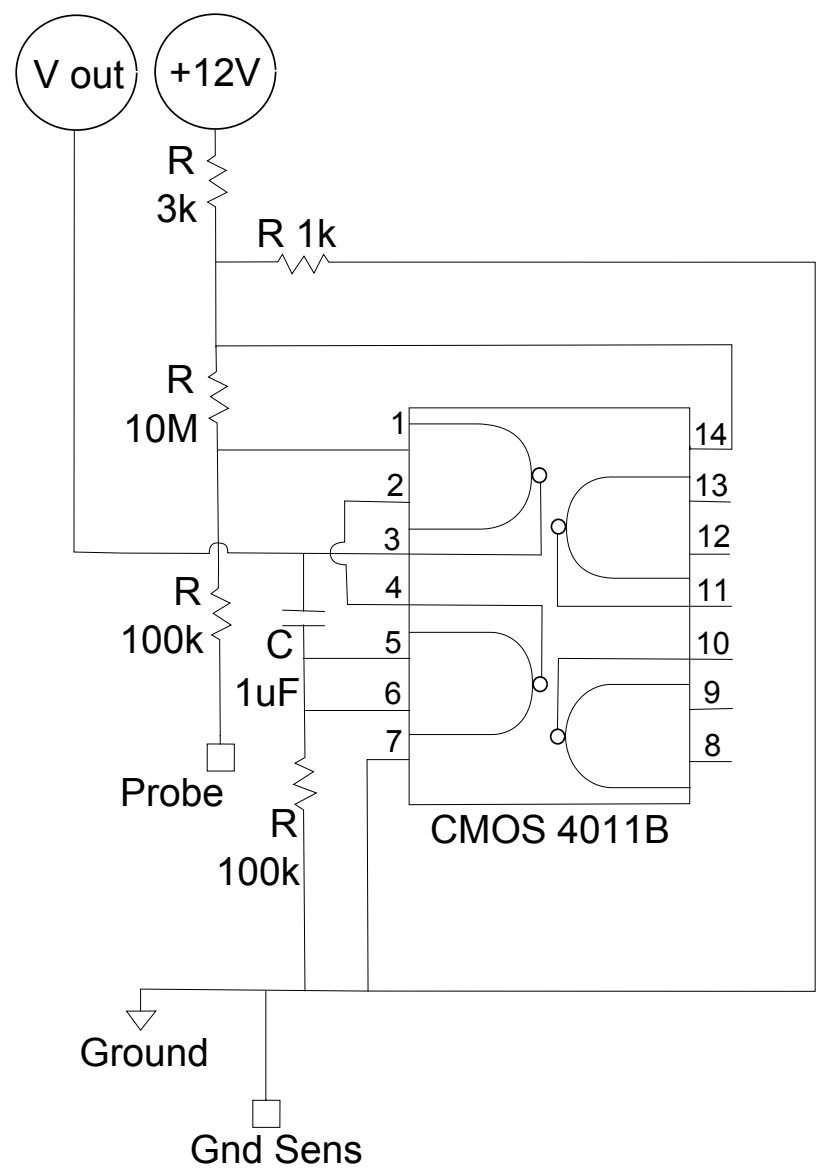

Figure A.1: Circuit schematic of water level sensor. This sensor is comprised of a probe connected to a circuit powered by a $12 \mathrm{~V}$ signal. When no conductive path is available from probe to ground the circuit remains open, but when a conductive path is available the circuit returns a $3 \mathrm{~V}$ signal. 


\section{Appendix B}

\section{LabView flow charts}

Each flow chart is constructed as follows: (1) the underlined sections correspond to a task the user must complete or an option that must be set manually, (2) the white boxes correspond to specific options the user must select to continue, and (3) the dark boxes correspond to a task completed within the LabVIEW software. 


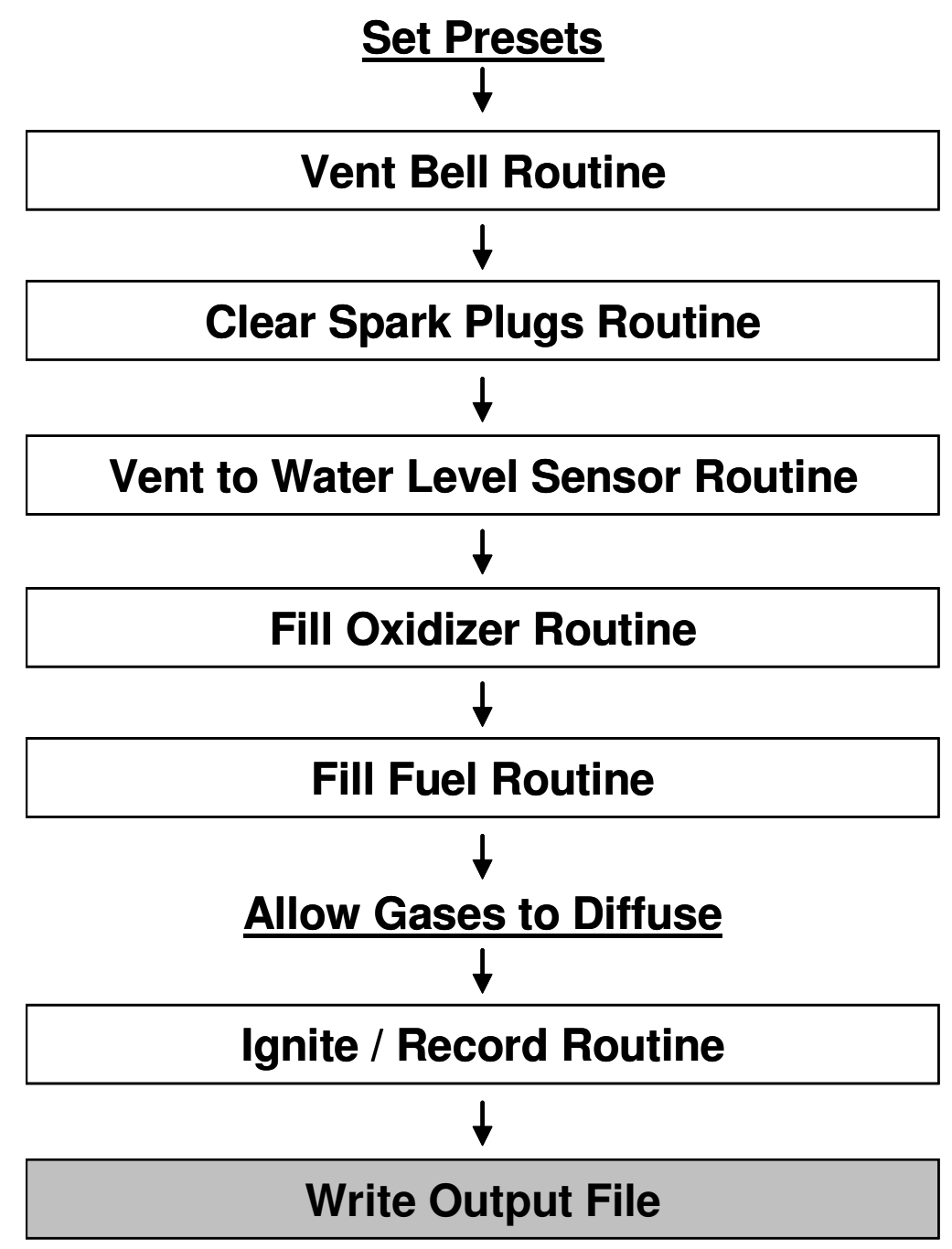

Figure B.1: The operational flow chart for firing the CSS with an air-hydrogen mixture. The underlined items refer to manual tasks, the items in white boxes refer to LabVIEW tasks that must be manually initiated, and the dark box refers to LabVIEW tasks that are automatically completed. 


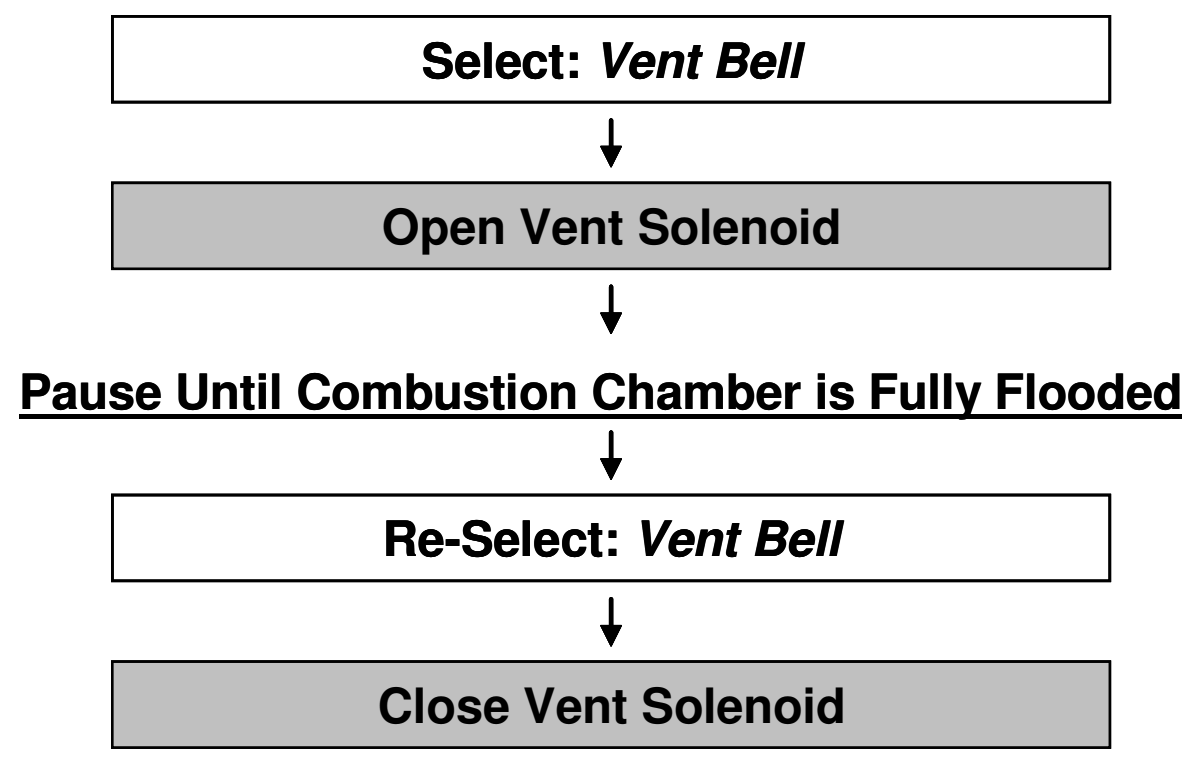

Figure B.2: Flow chart of LabVIEW user interface for Vent Routine. 


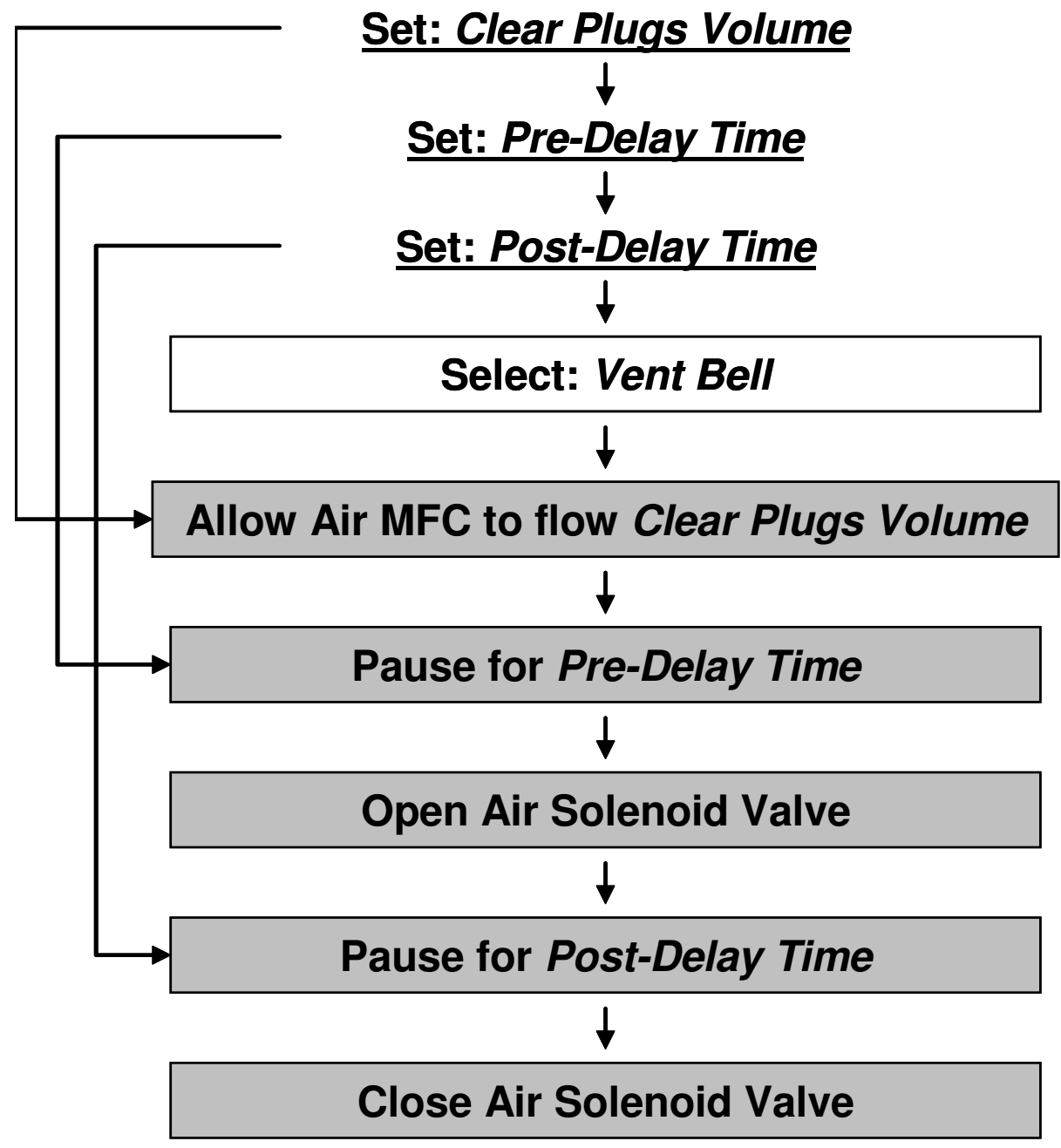

Figure B.3: Flow chart of LabVIEW user interface for Clear Plugs Routine. 


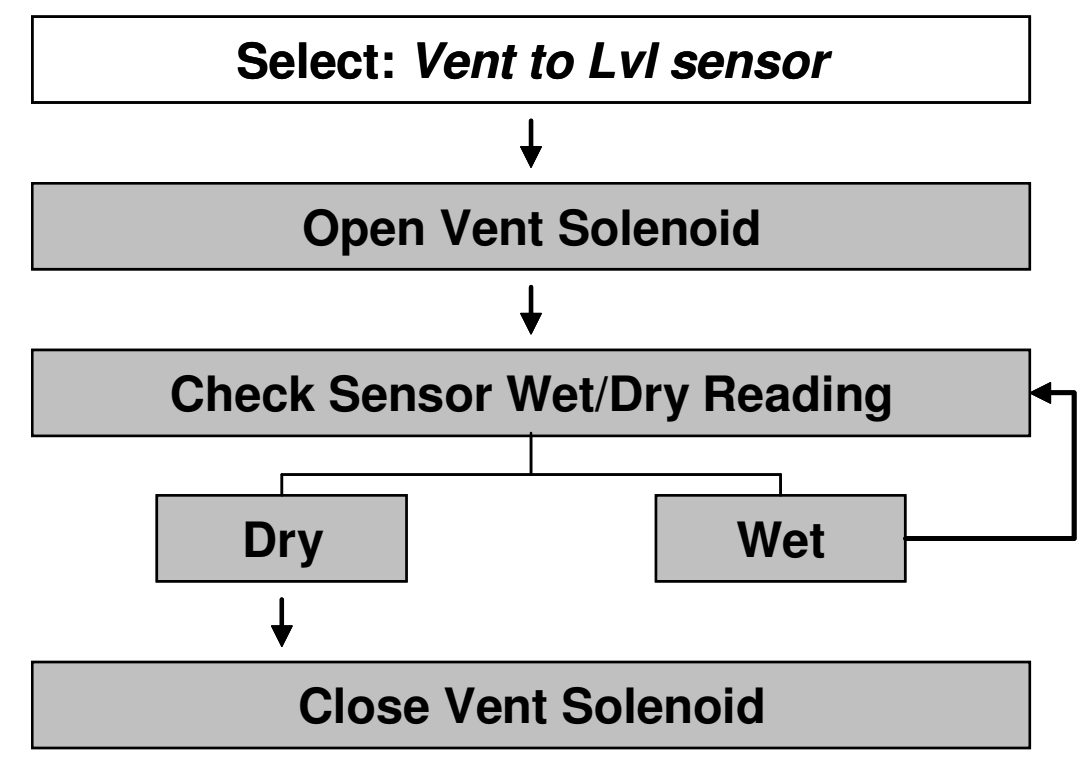

Figure B.4: Flow chart of LabVIEW user interface for Water Level Sensor Routine.

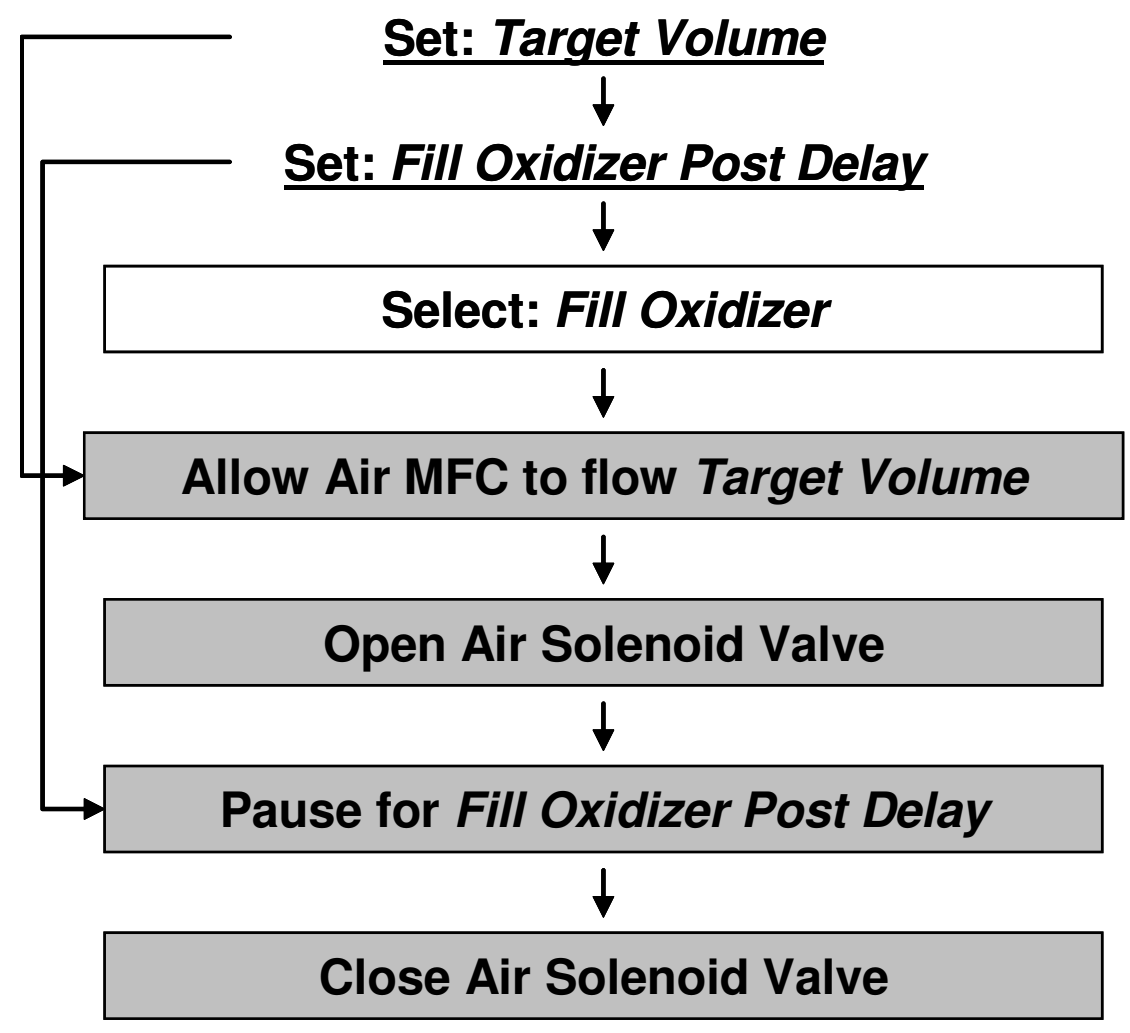

Figure B.5: Flow chart of LabVIEW user interface for Fill Oxidizer Routine. 


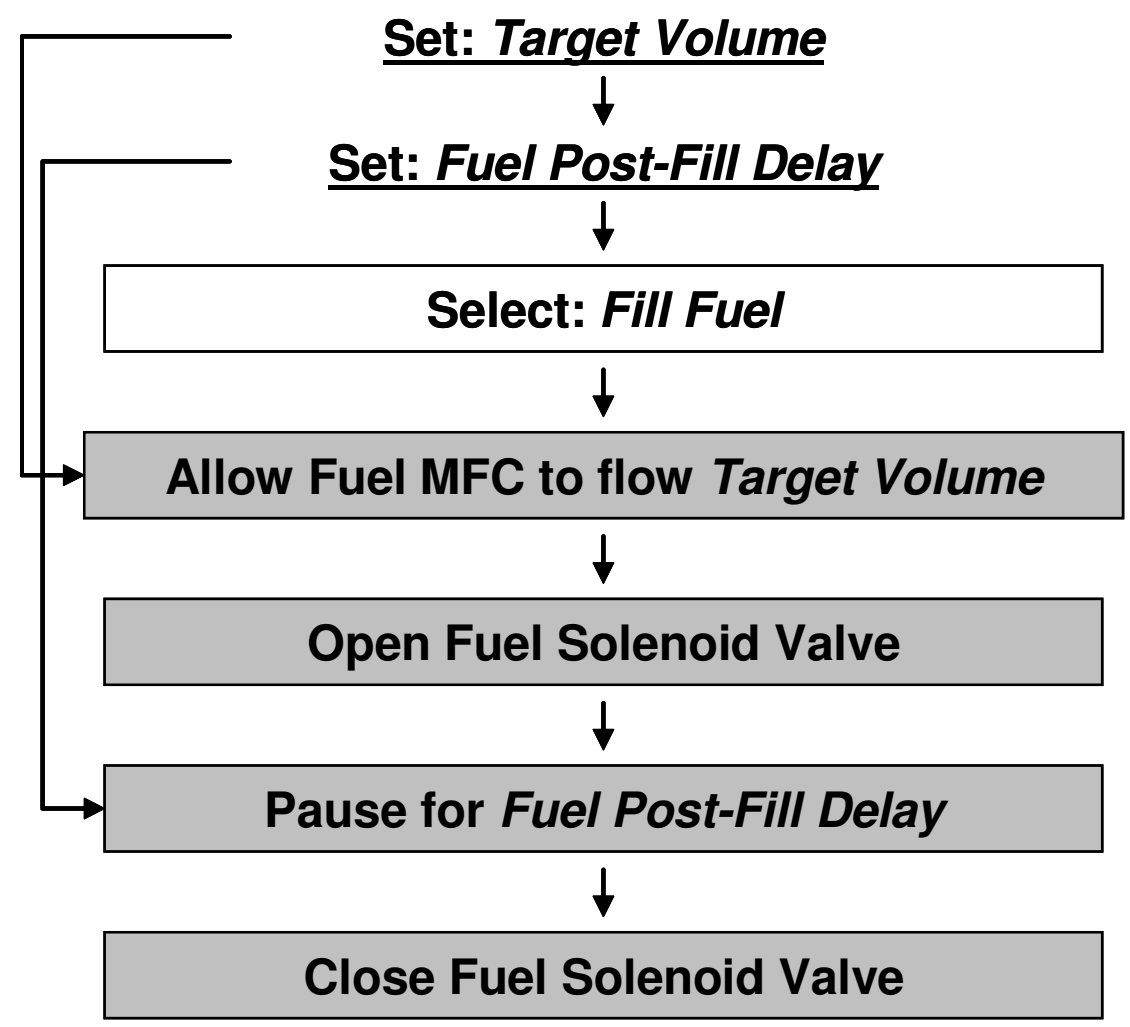

Figure B.6: Flow chart of LabVIEW user interface for Fill Fuel Routine. 


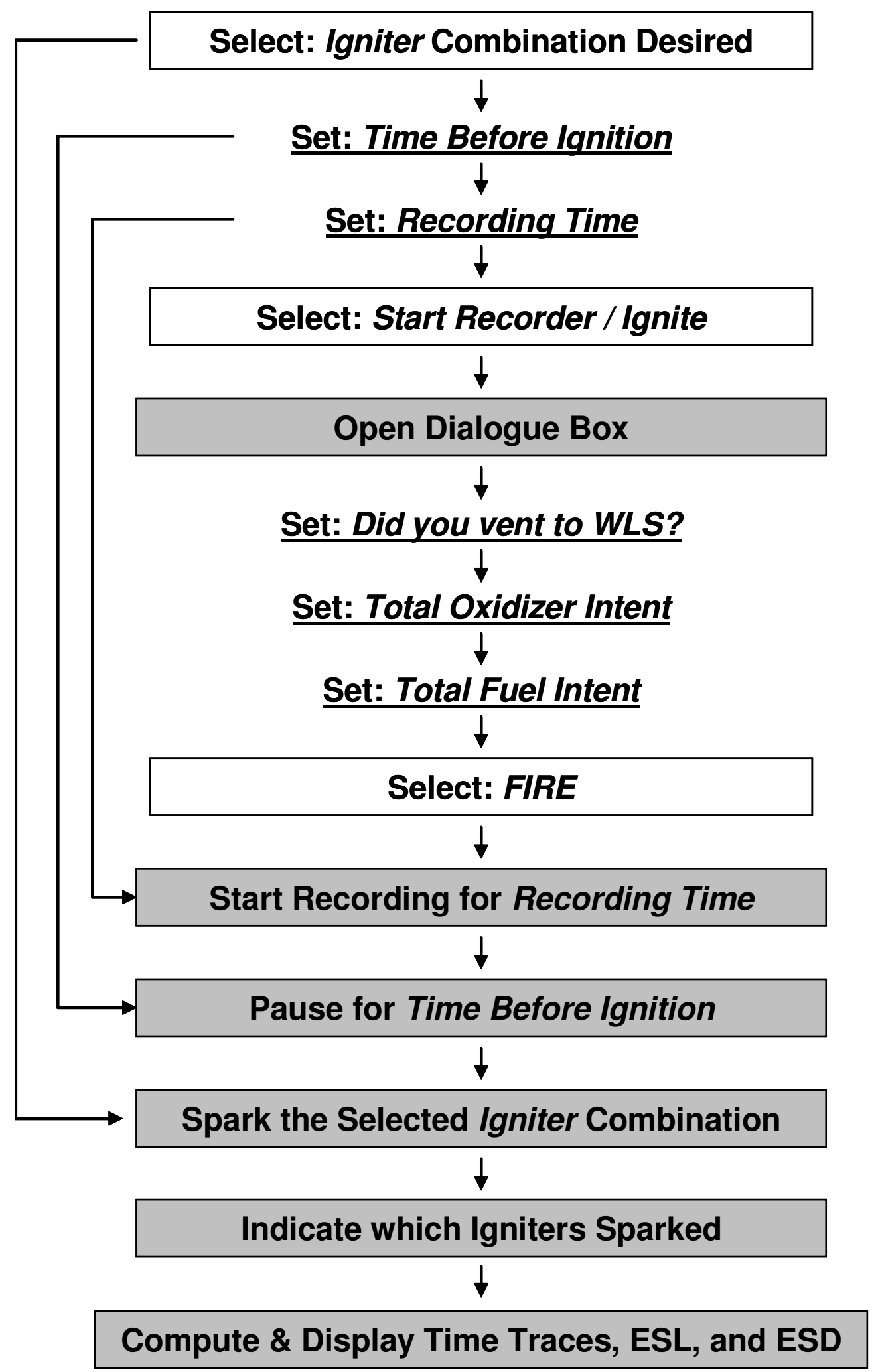

Figure B.7: Flow chart of LabVIEW user interface for Ignite/Record Routine. 


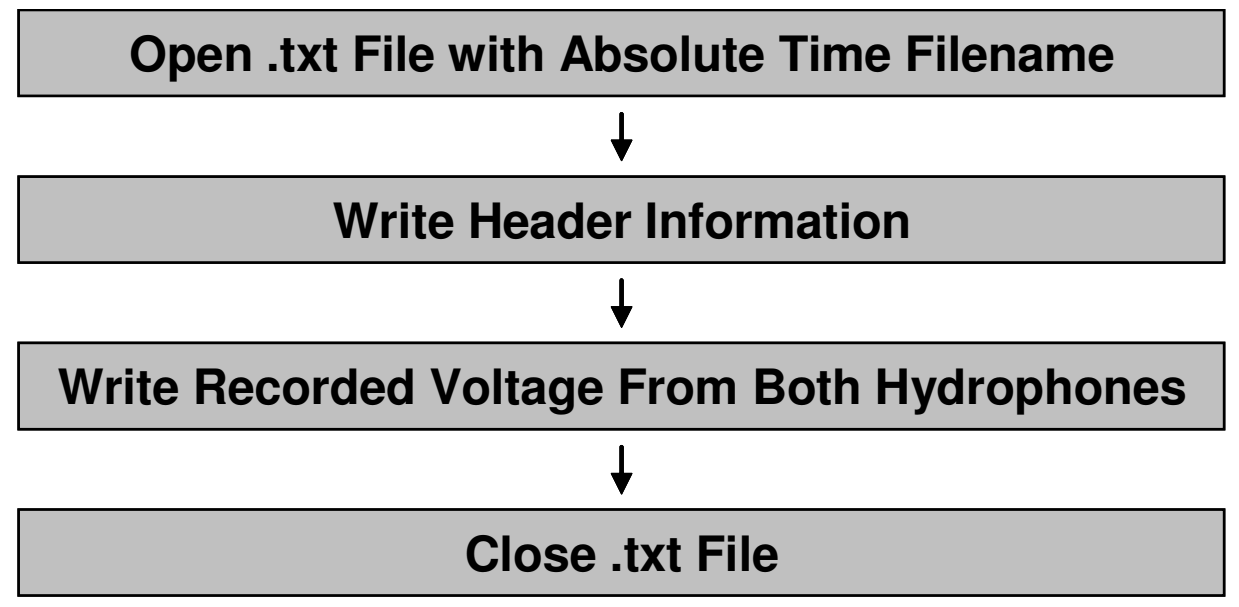

Figure B.8: Flow chart of LabVIEW user interface for Write Output Routine. 


\section{Appendix C}

\section{CSS Time Series and Spectra}

All the free-field recordings of CSS shots obtained during this work are presented in Appendix C.

\section{C.1 Air-Hydrogen, 24" Combustion Chamber}

Presented are time series and spectra of each parameter set described by the airhydrogen mixture fired in the 24" combustion chamber for various volumes and depths. 

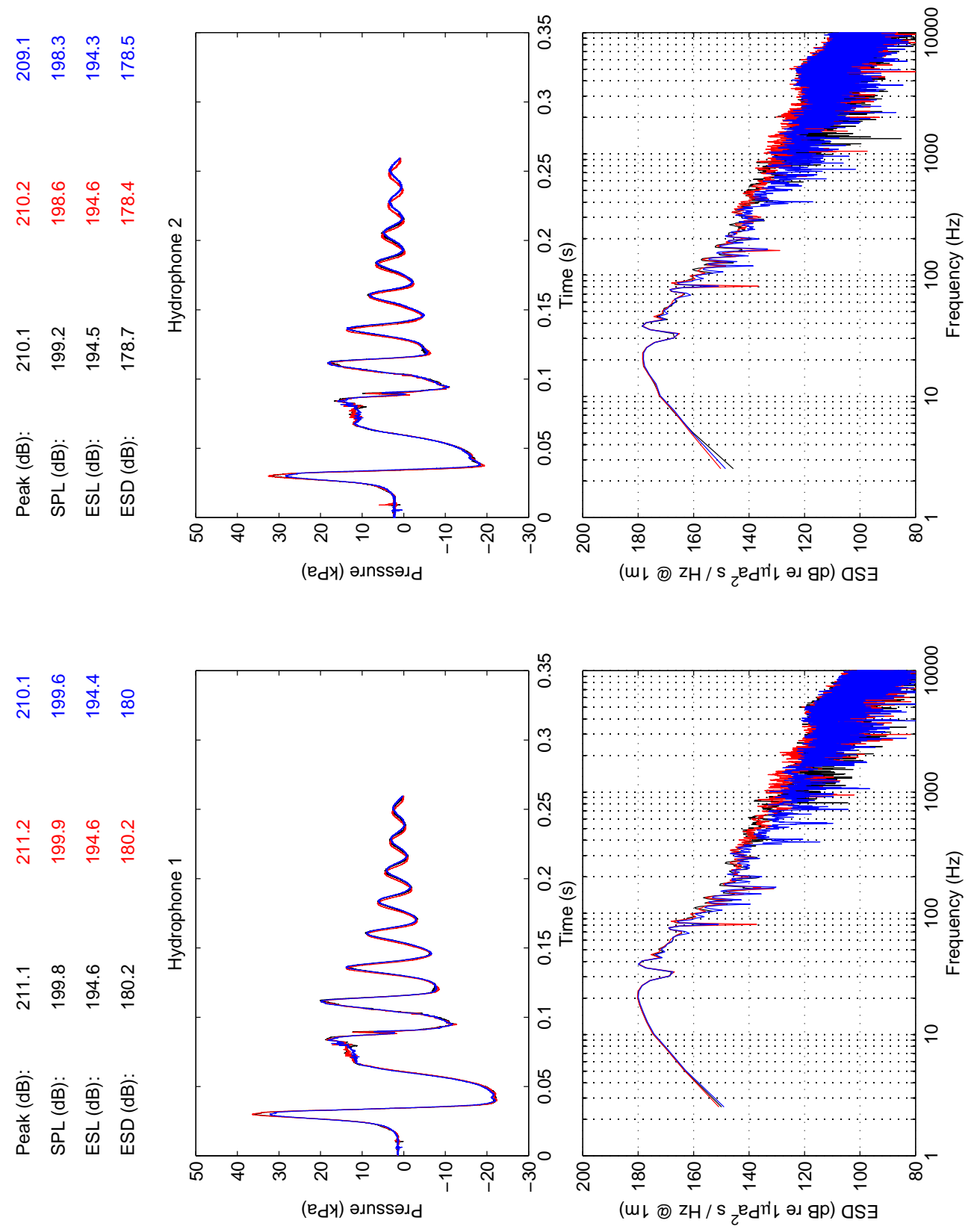

Figure C.1: Time series and spectra of the parameter set described by 9 L (STP) of the airhydrogen mixture fired in the $24 "$ chamber at a depth of $5 \mathrm{~m}$. 

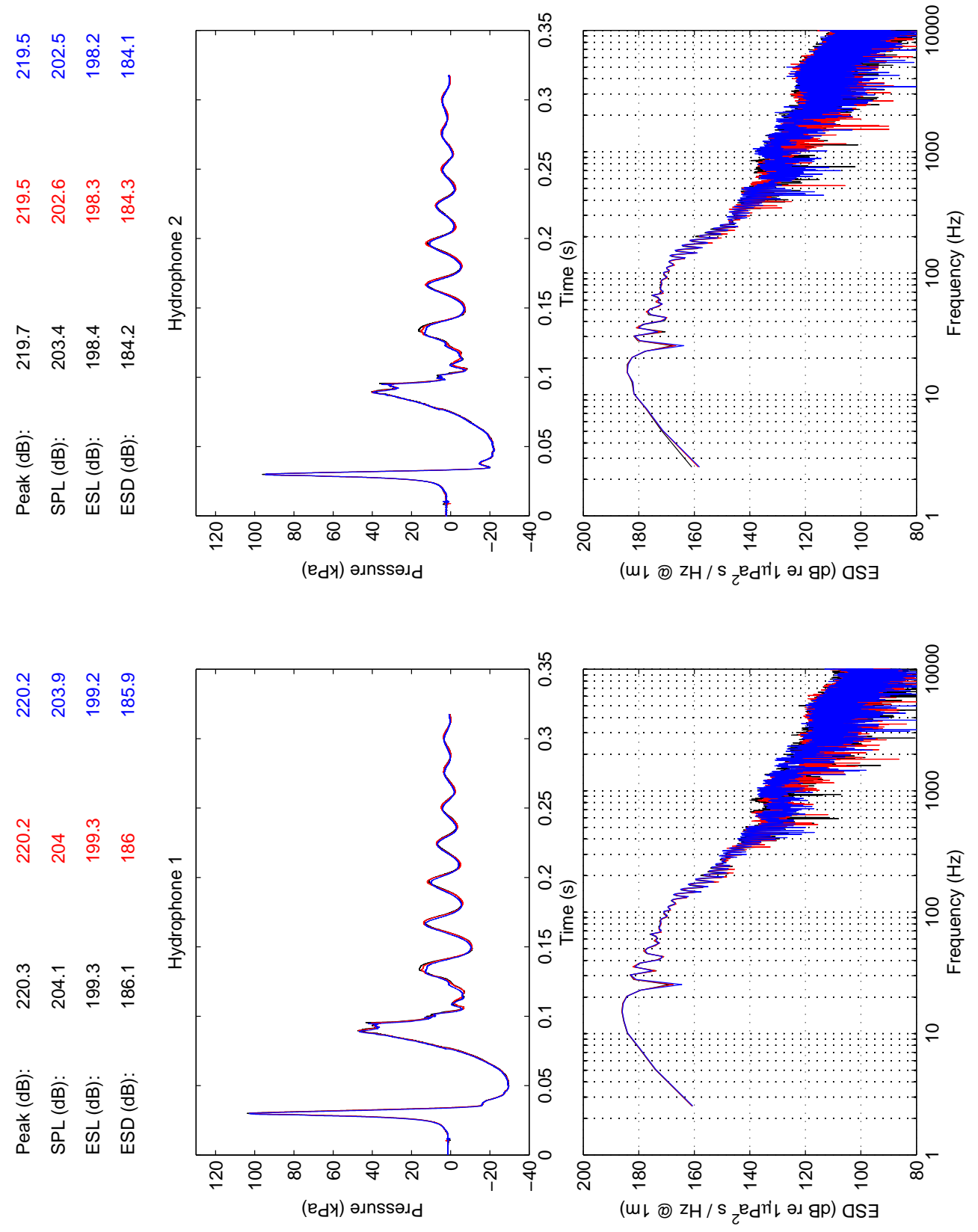

Figure C.2: Time series and spectra of the parameter set described by $18 \mathrm{~L}$ (STP) of the air-hydrogen mixture fired in the 24 " chamber at a depth of $5 \mathrm{~m}$. 

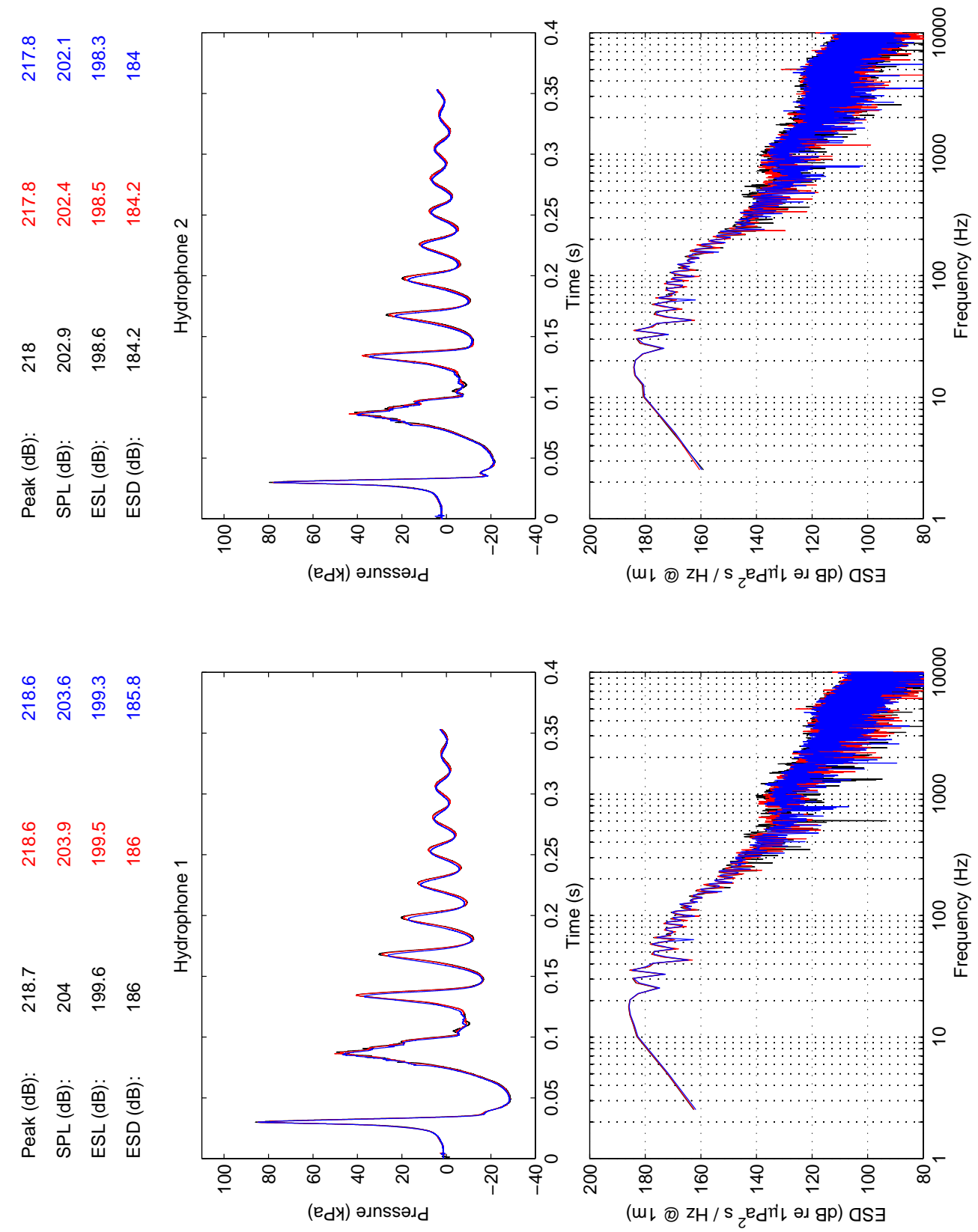

Figure C.3: Time series and spectra of the parameter set described by $27 \mathrm{~L}$ (STP) of the air-hydrogen mixture fired in the 24 " chamber at a depth of $5 \mathrm{~m}$. 

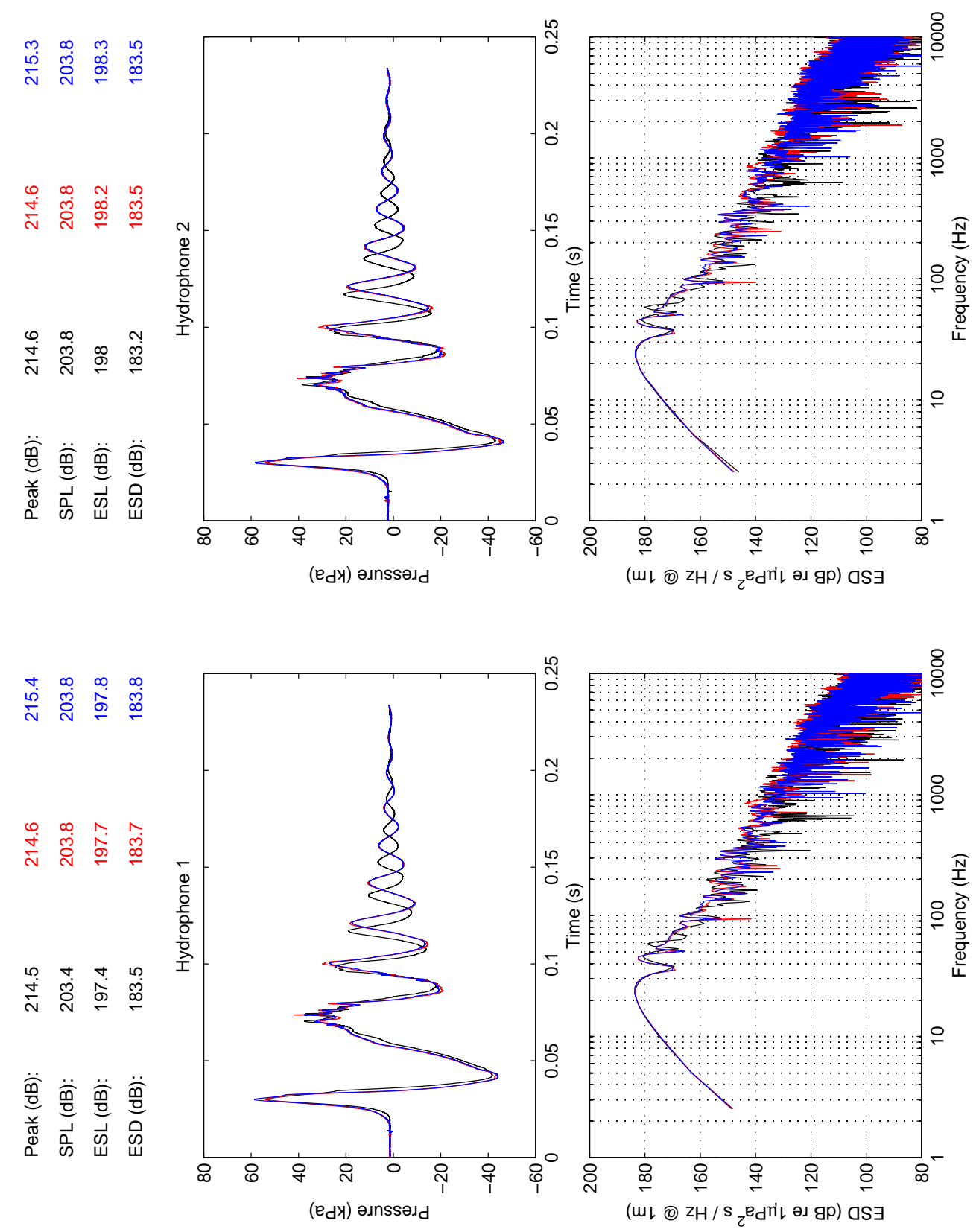

Figure C.4: Time series and spectra of the parameter set described by $12 \mathrm{~L}$ (STP) of the air-hydrogen mixture fired in the $24 "$ chamber at a depth of $10 \mathrm{~m}$. 

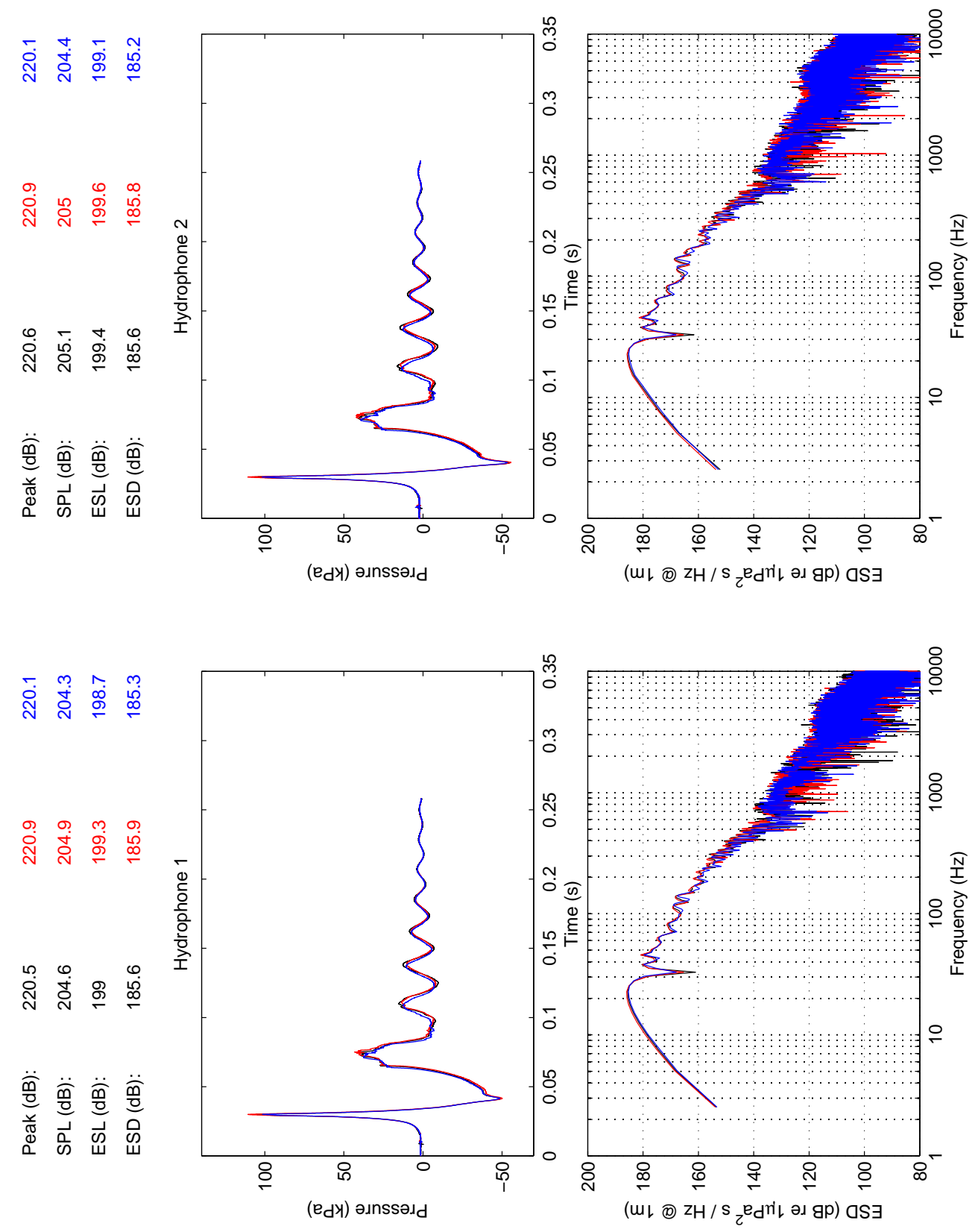

Figure C.5: Time series and spectra of the parameter set described by $20 \mathrm{~L}$ (STP) of the air-hydrogen mixture fired in the $24 "$ chamber at a depth of $10 \mathrm{~m}$. 

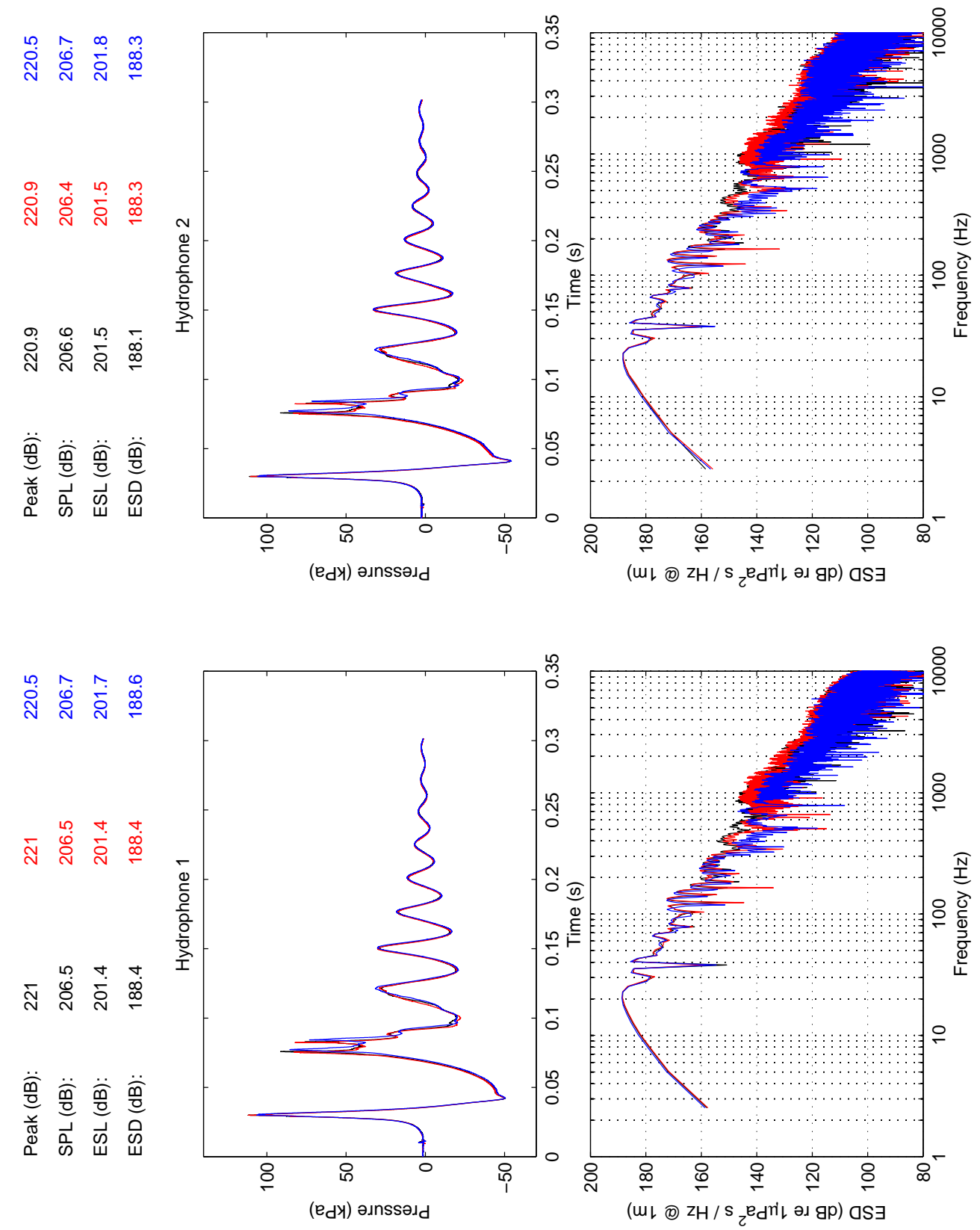

Figure C.6: Time series and spectra of the parameter set described by $28 \mathrm{~L}$ (STP) of the air-hydrogen mixture fired in the $24 "$ chamber at a depth of $10 \mathrm{~m}$. 

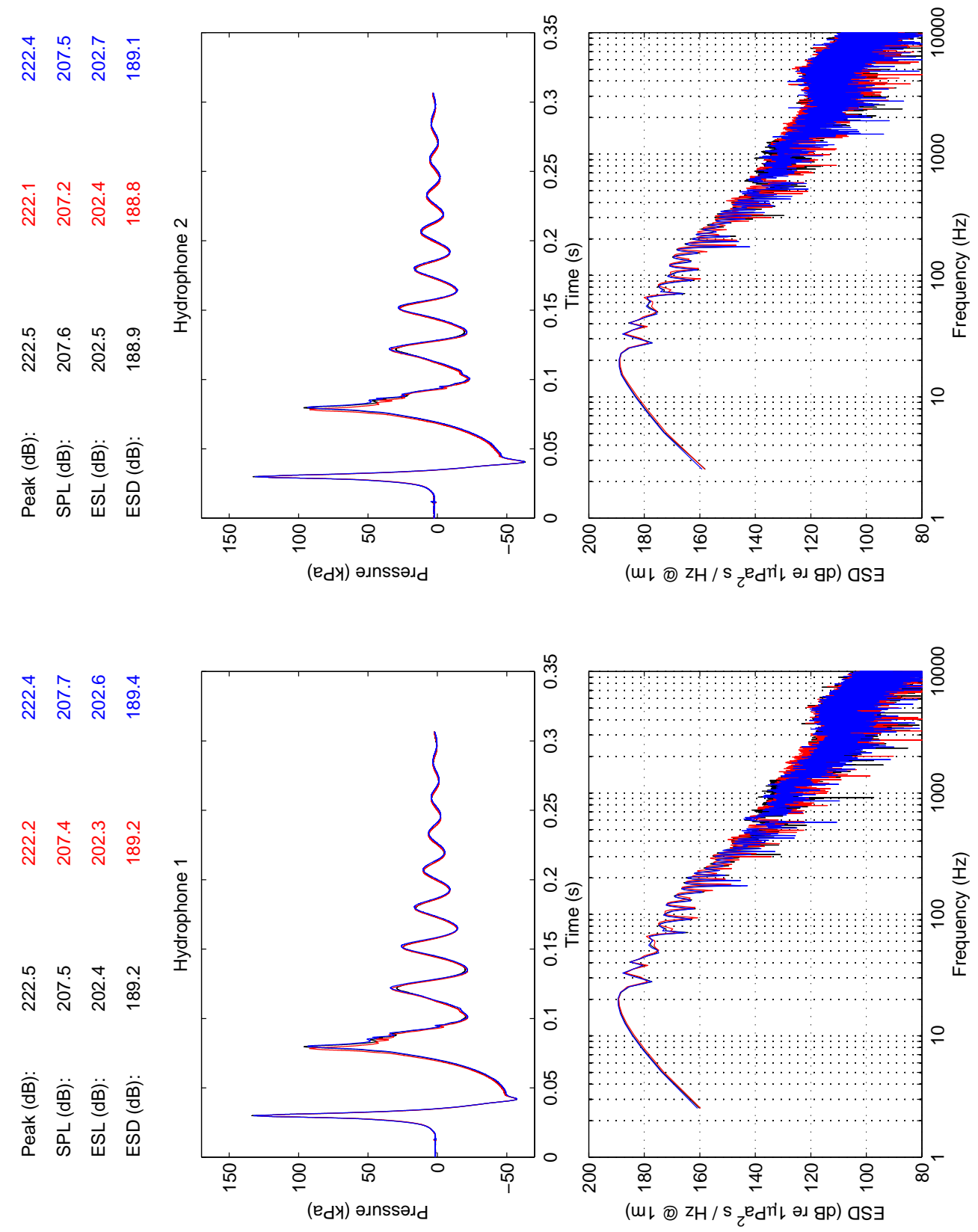

Figure C.7: Time series and spectra of the parameter set described by $36 \mathrm{~L}$ (STP) of the air-hydrogen mixture fired in the $24 "$ chamber at a depth of $10 \mathrm{~m}$. 


\section{C.2 Air-Hydrogen, 36" Combustion Chamber}

Presented are time series and spectra of each parameter set described by the airhydrogen mixture fired in the 36 " combustion chamber for various volumes and depths. 

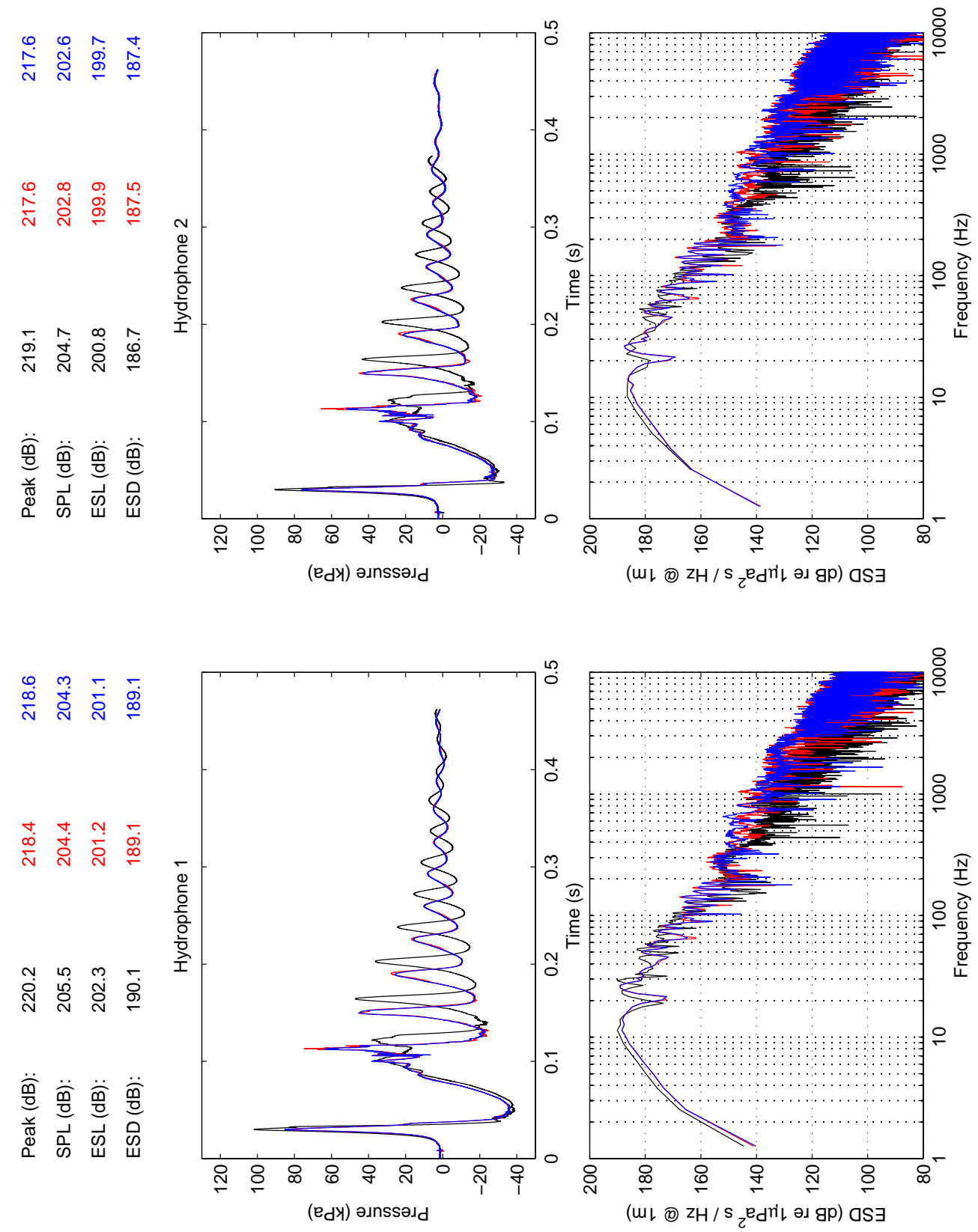

Figure C.8: Time series and spectra of the parameter set described by $30 \mathrm{~L}$ (STP) of the air-hydrogen mixture fired in the 36 " chamber at a depth of $5 \mathrm{~m}$. 

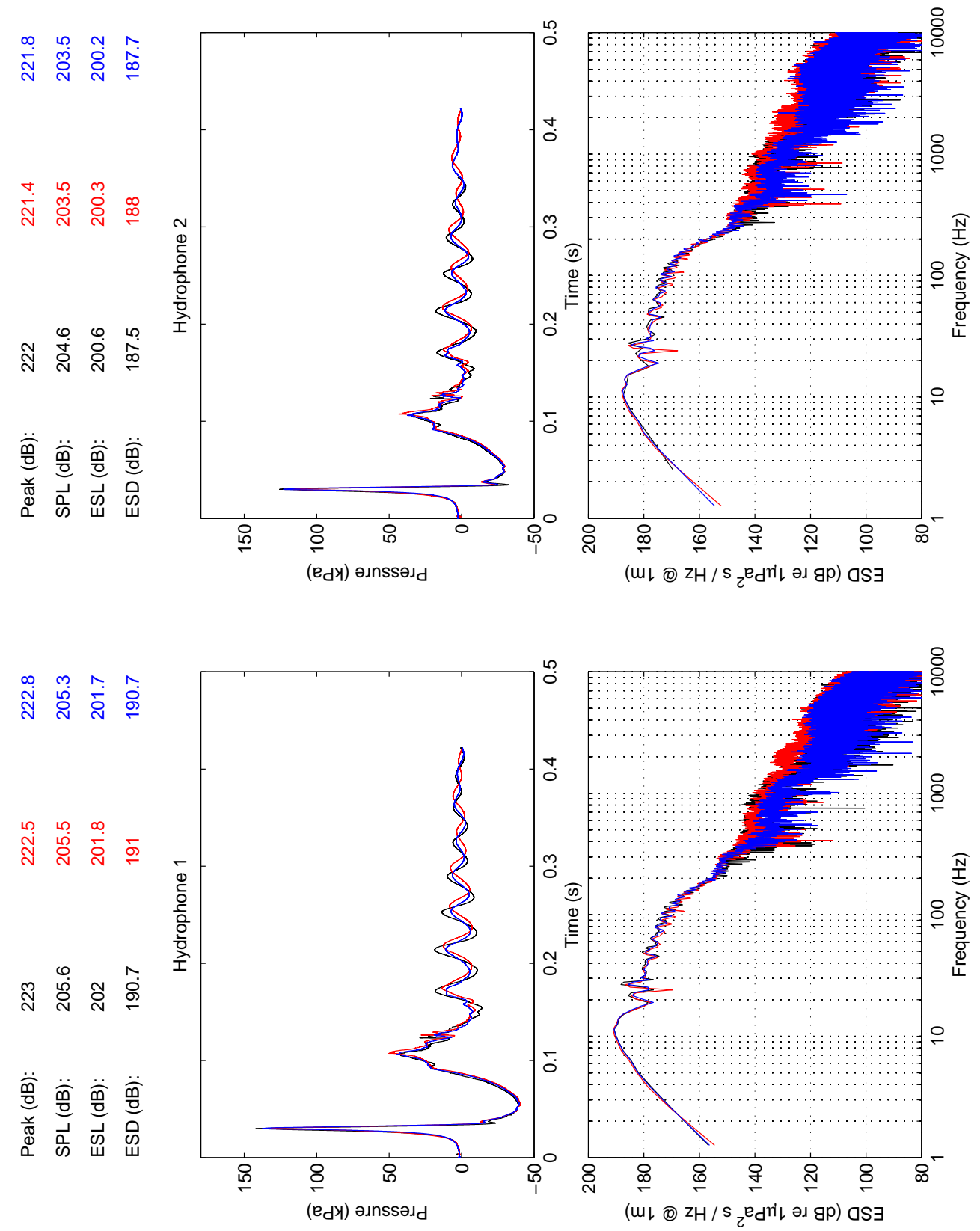

Figure C.9: Time series and spectra of the parameter set described by $60 \mathrm{~L}$ (STP) of the air-hydrogen mixture fired in the $36 "$ chamber at a depth of $5 \mathrm{~m}$. 

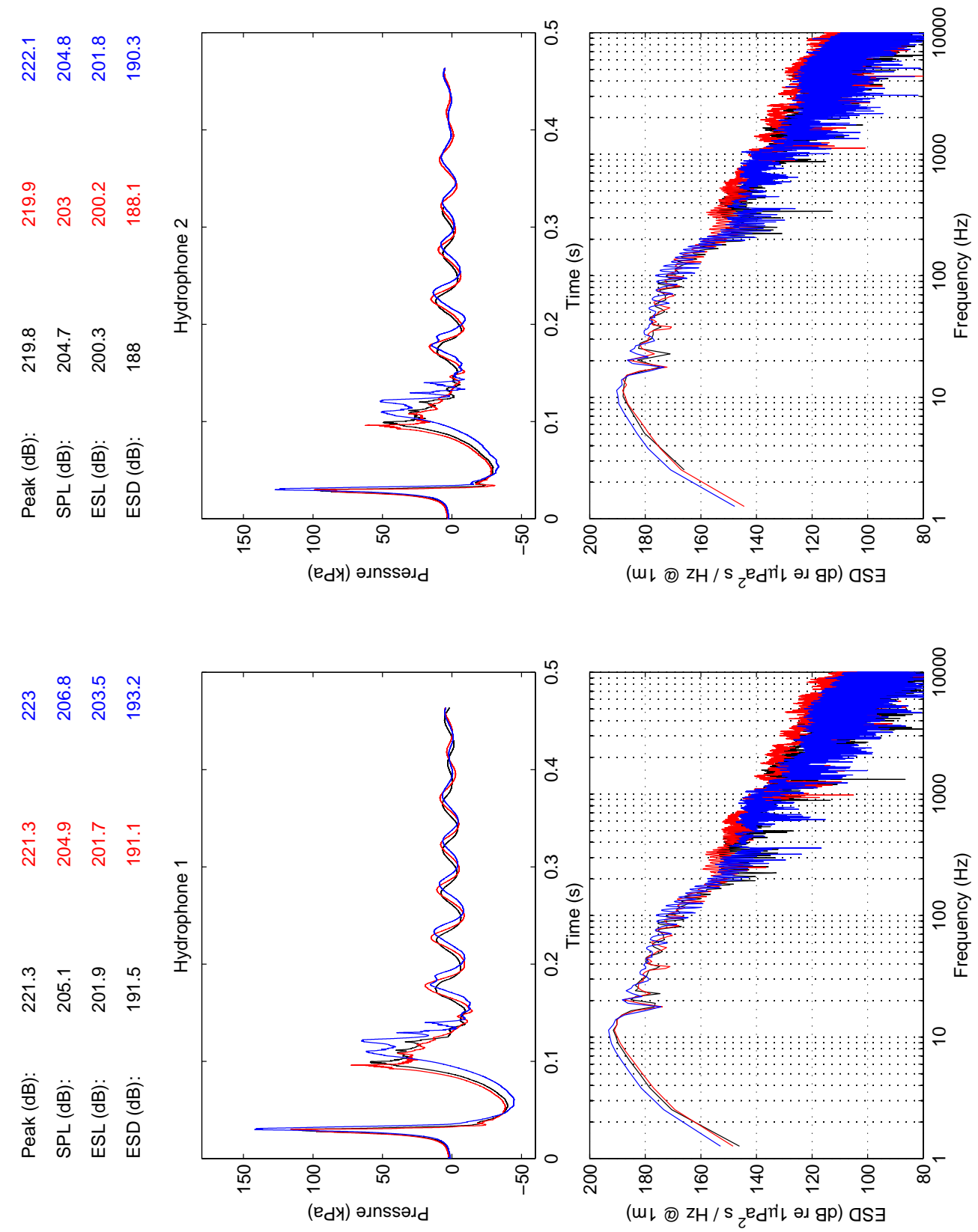

Figure C.10: Time series and spectra of the parameter set described by $90 \mathrm{~L}$ (STP) of the air-hydrogen mixture fired in the 36 " chamber at a depth of $5 \mathrm{~m}$. 

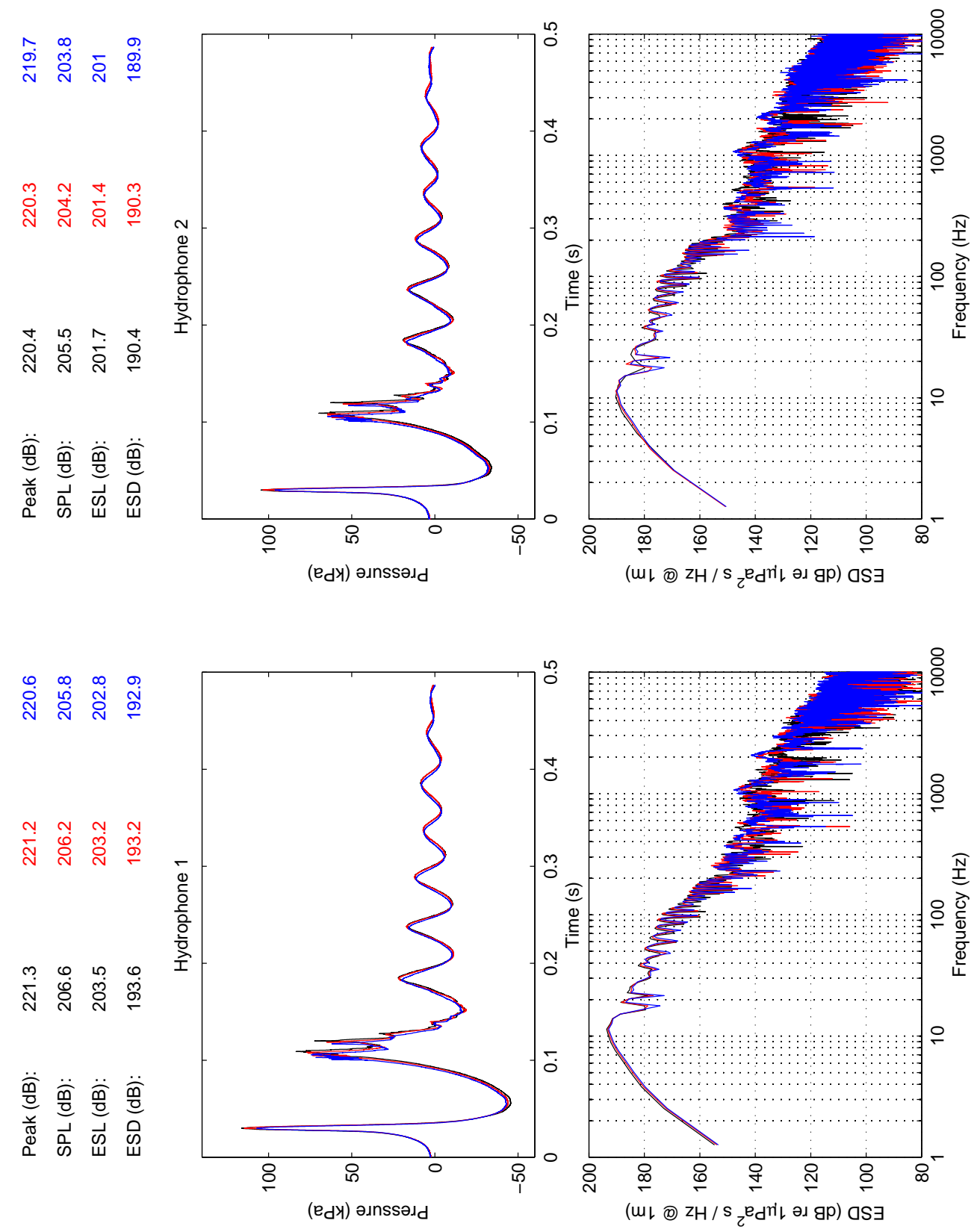

Figure C.11: Time series and spectra of the parameter set described by $120 \mathrm{~L}$ (STP) of the air-hydrogen mixture fired in the $36^{\prime \prime}$ chamber at a depth of $5 \mathrm{~m}$. 

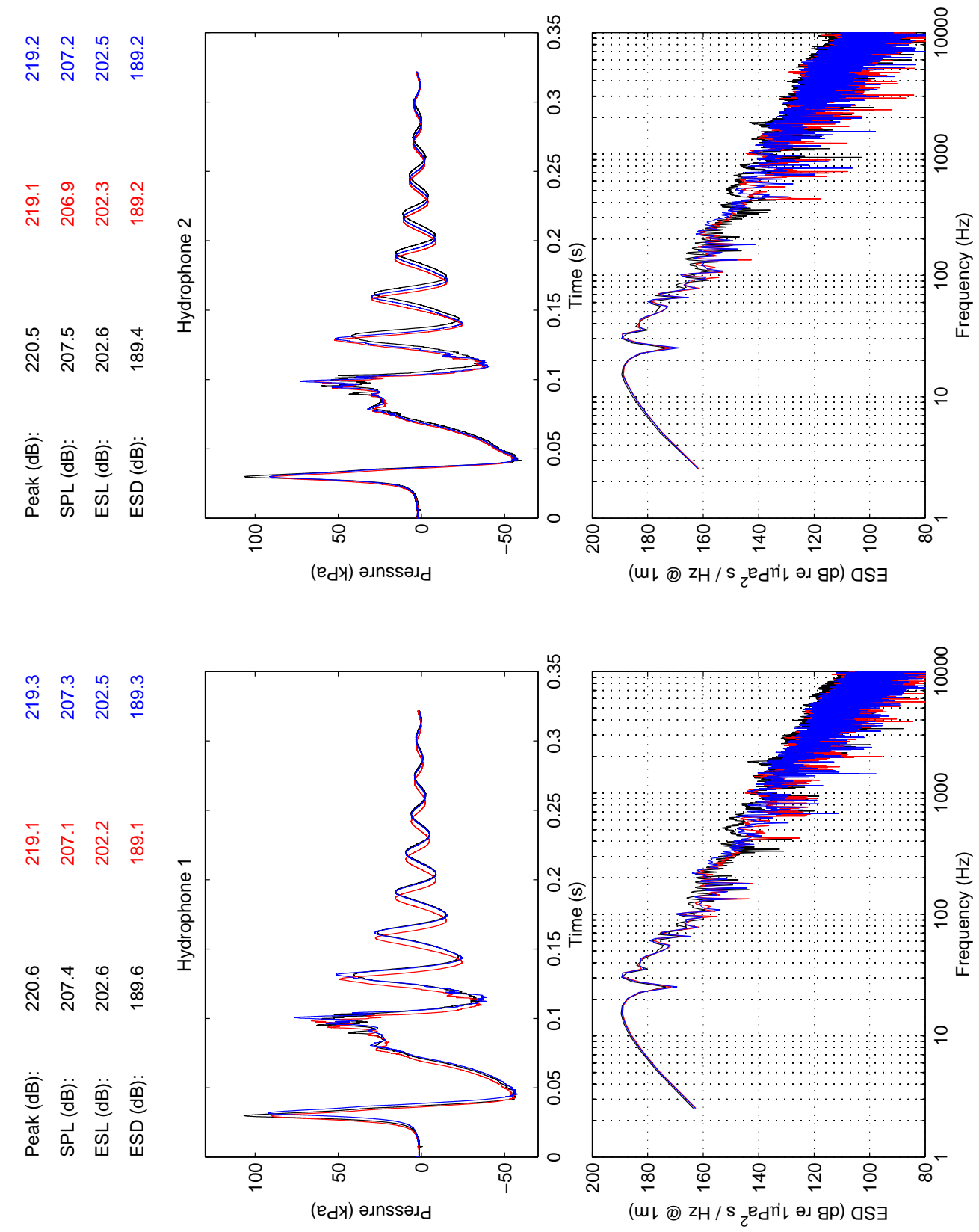

Figure C.12: Time series and spectra of the parameter set described by $40 \mathrm{~L}$ (STP) of the air-hydrogen mixture fired in the $36 "$ chamber at a depth of $10 \mathrm{~m}$. 

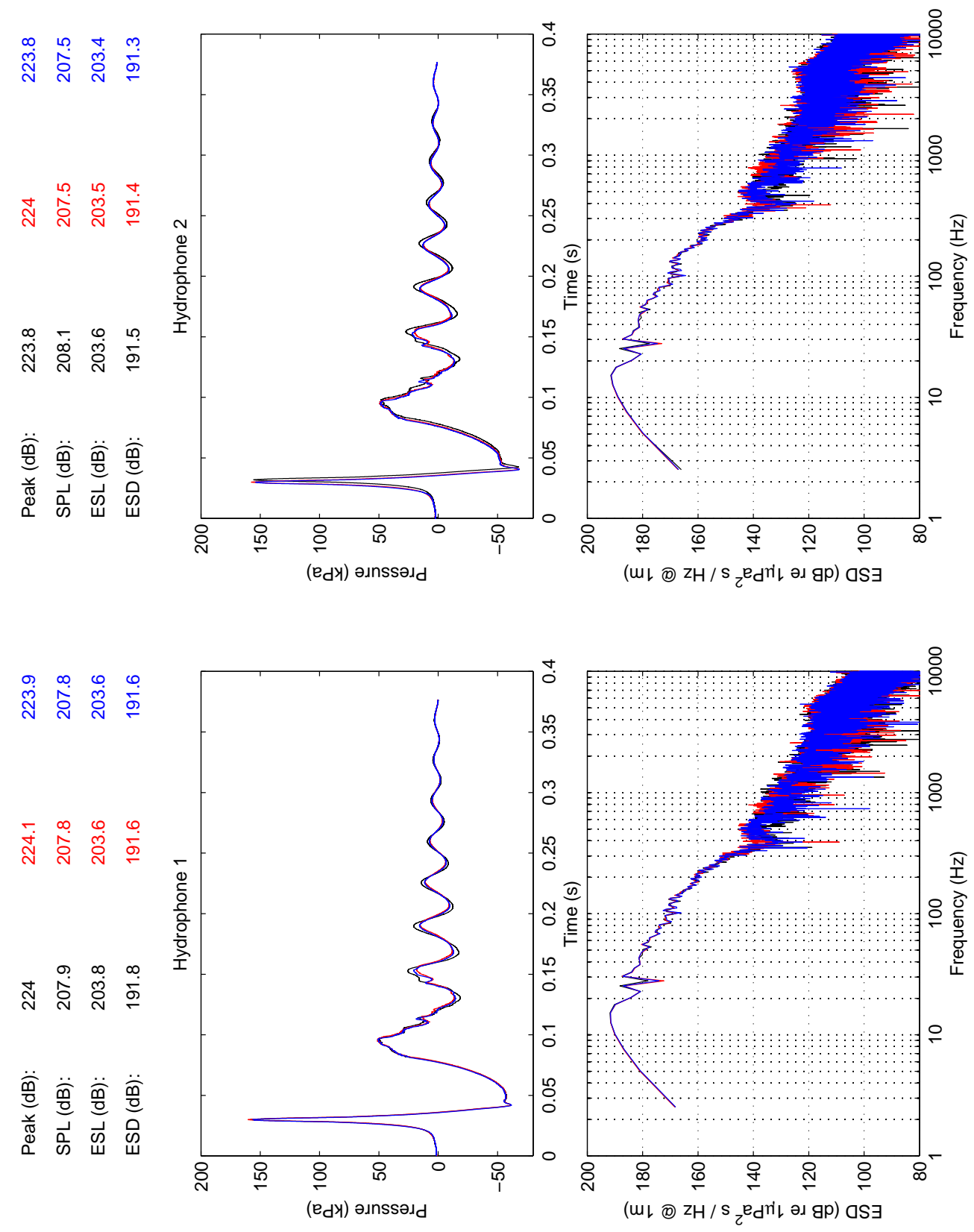

Figure C.13: Time series and spectra of the parameter set described by $80 \mathrm{~L}$ (STP) of the air-hydrogen mixture fired in the $36 "$ chamber at a depth of $10 \mathrm{~m}$. 

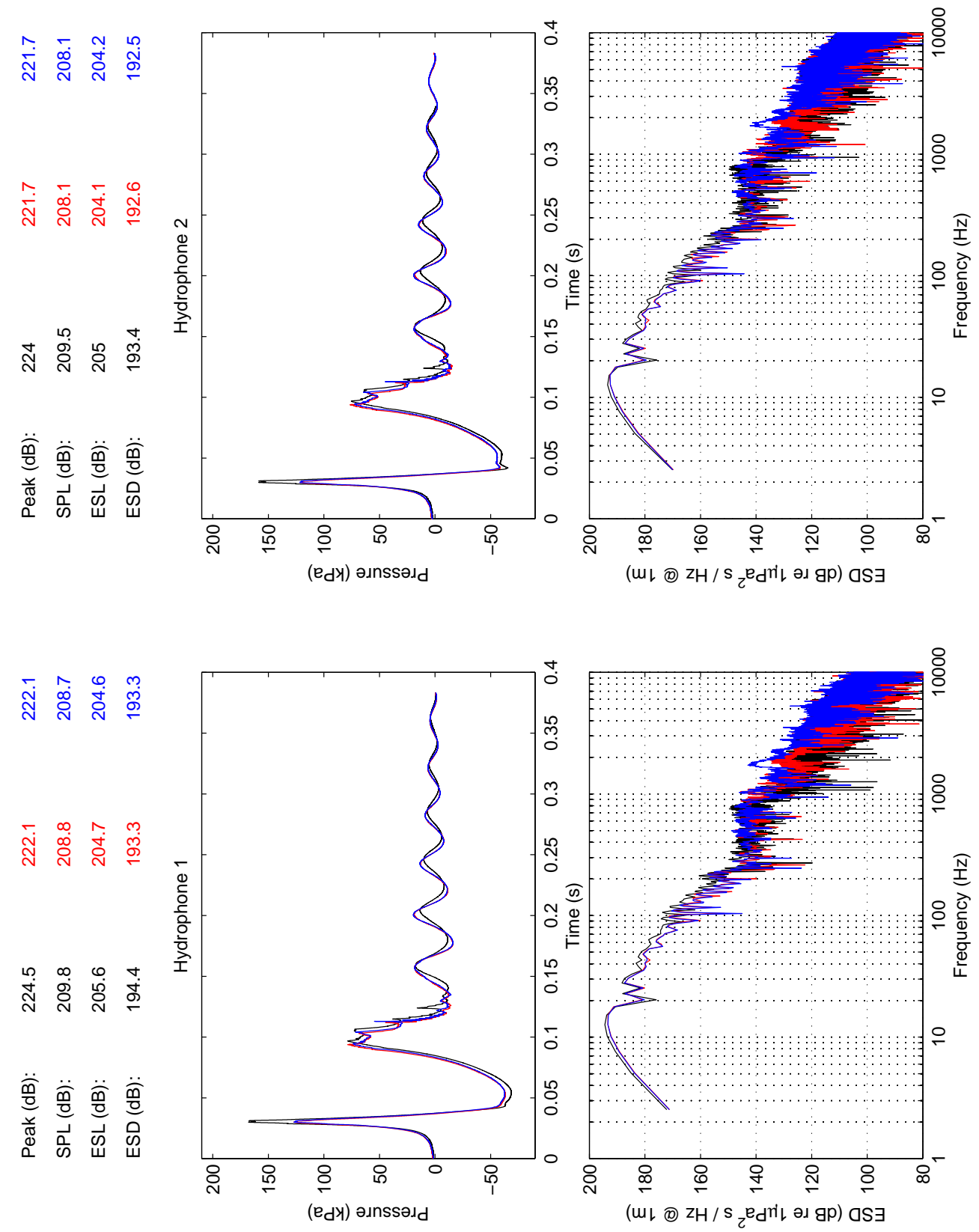

Figure C.14: Time series and spectra of the parameter set described by $120 \mathrm{~L}$ (STP) of the air-hydrogen mixture fired in the $36 "$ chamber at a depth of $10 \mathrm{~m}$. 

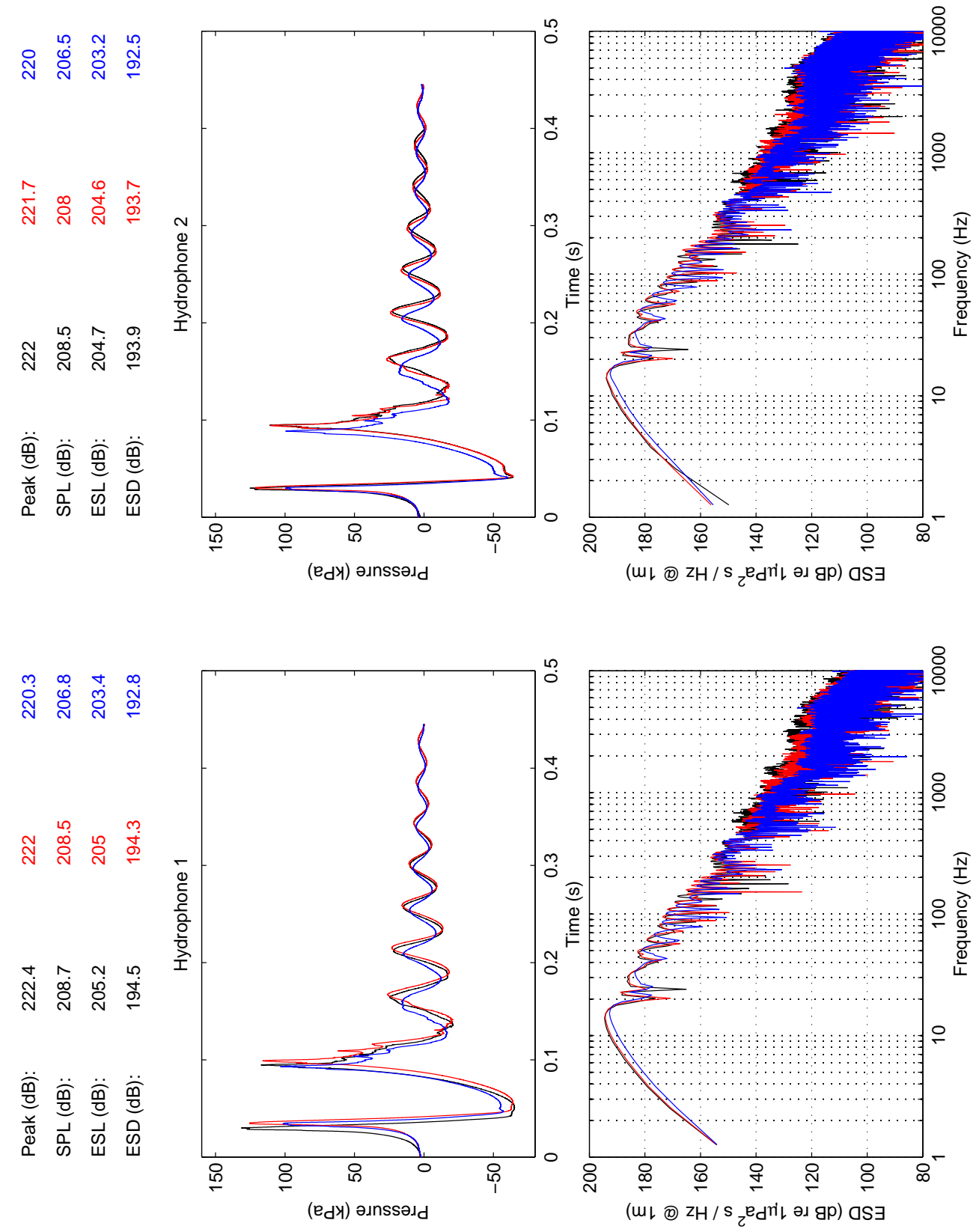

Figure C.15: Time series and spectra of the parameter set described by $160 \mathrm{~L}$ (STP) of the air-hydrogen mixture fired in the $36 "$ chamber at a depth of $10 \mathrm{~m}$. 


\section{C.3 Air-Hydrogen, 48" Combustion Chamber}

Presented are time series and spectra of each parameter set described by the airhydrogen mixture fired in the $48^{\prime \prime}$ combustion chamber for various volumes and depths. 

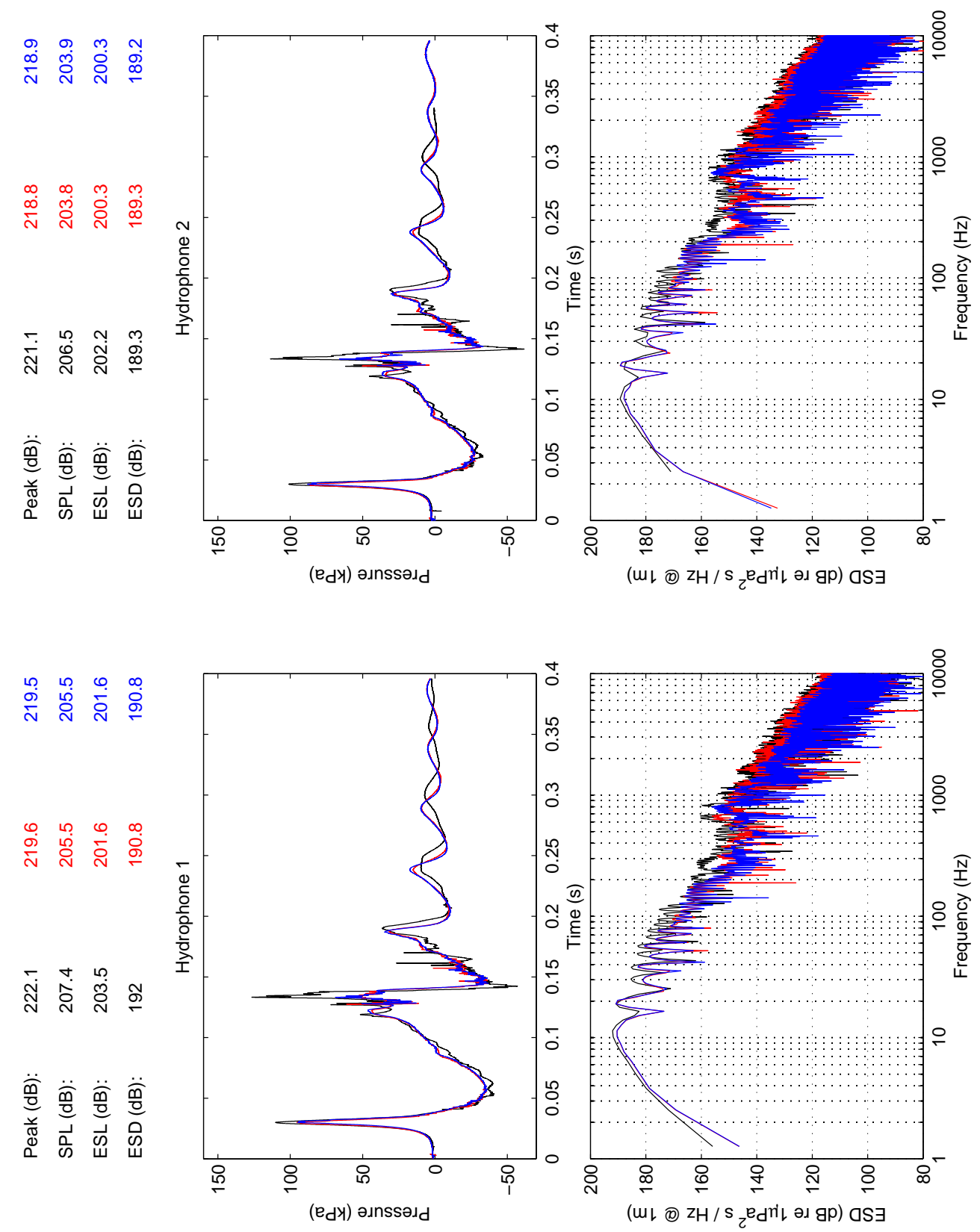

Figure C.16: Time series and spectra of the parameter set described by $60 \mathrm{~L}$ (STP) of the air-hydrogen mixture fired in the 48 " chamber at a depth of $5 \mathrm{~m}$. 

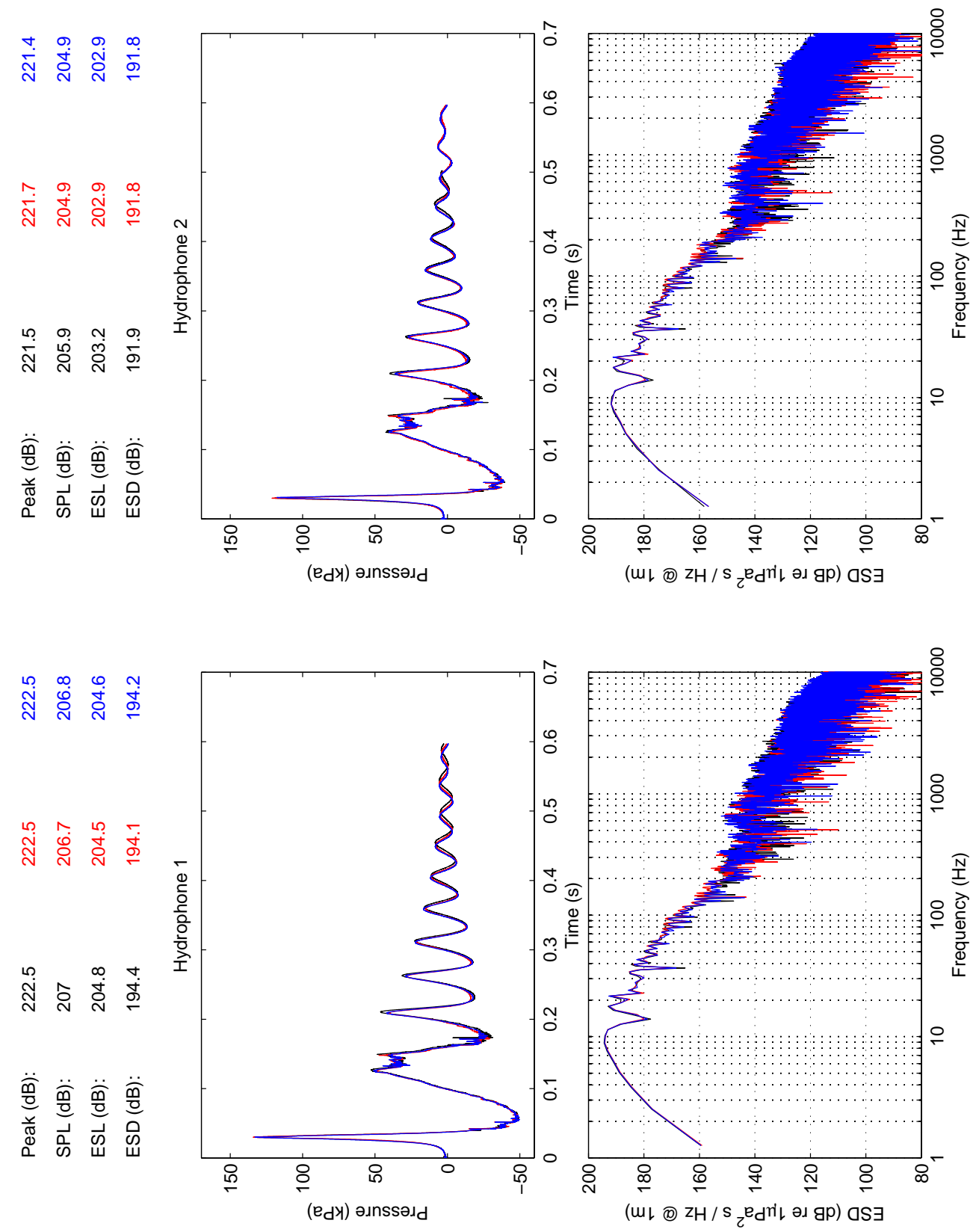

Figure C.17: Time series and spectra of the parameter set described by $120 \mathrm{~L}$ (STP) of the air-hydrogen mixture fired in the 48 " chamber at a depth of $5 \mathrm{~m}$. 

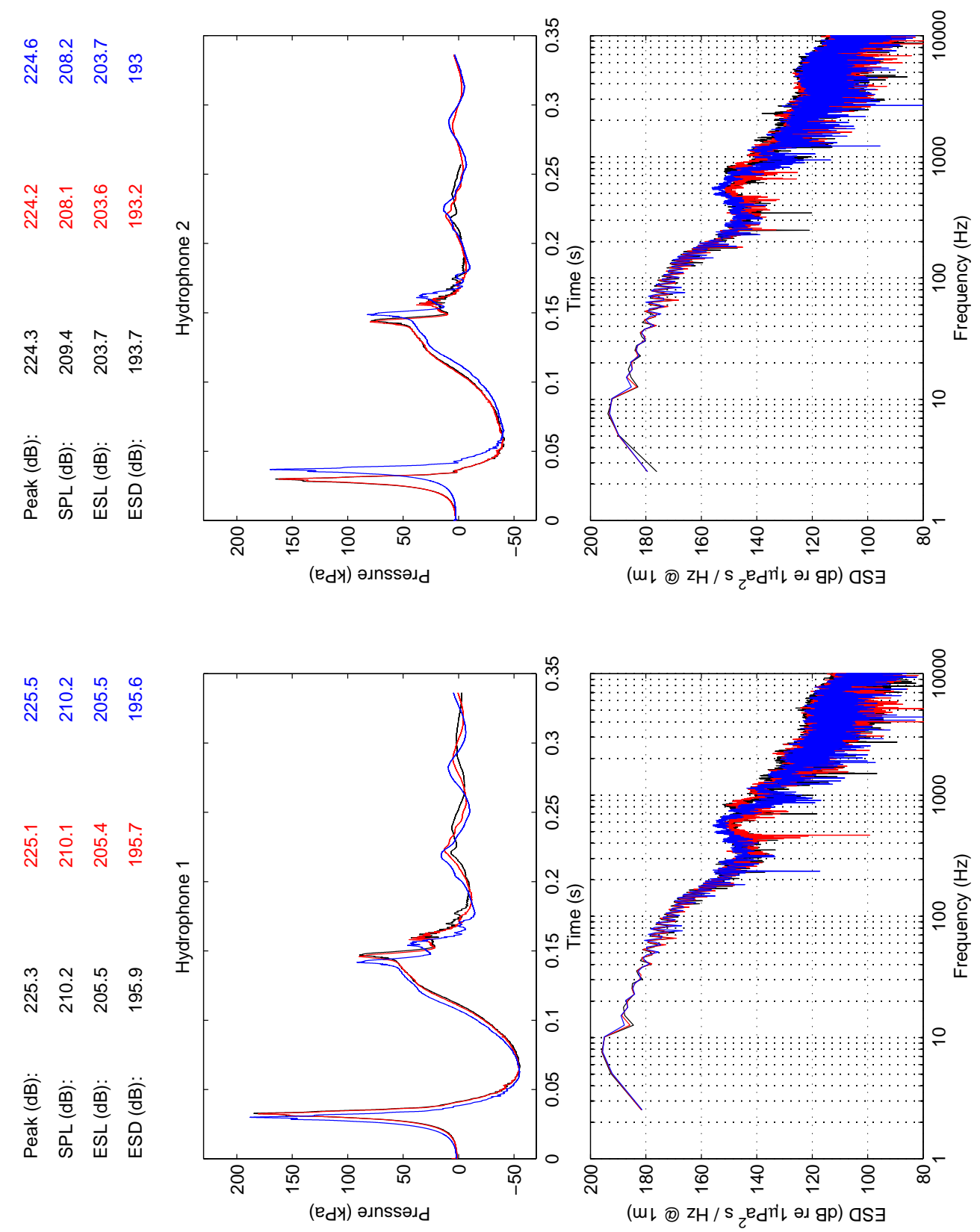

Figure C.18: Time series and spectra of the parameter set described by $180 \mathrm{~L}$ (STP) of the air-hydrogen mixture fired in the 48 " chamber at a depth of $5 \mathrm{~m}$. 

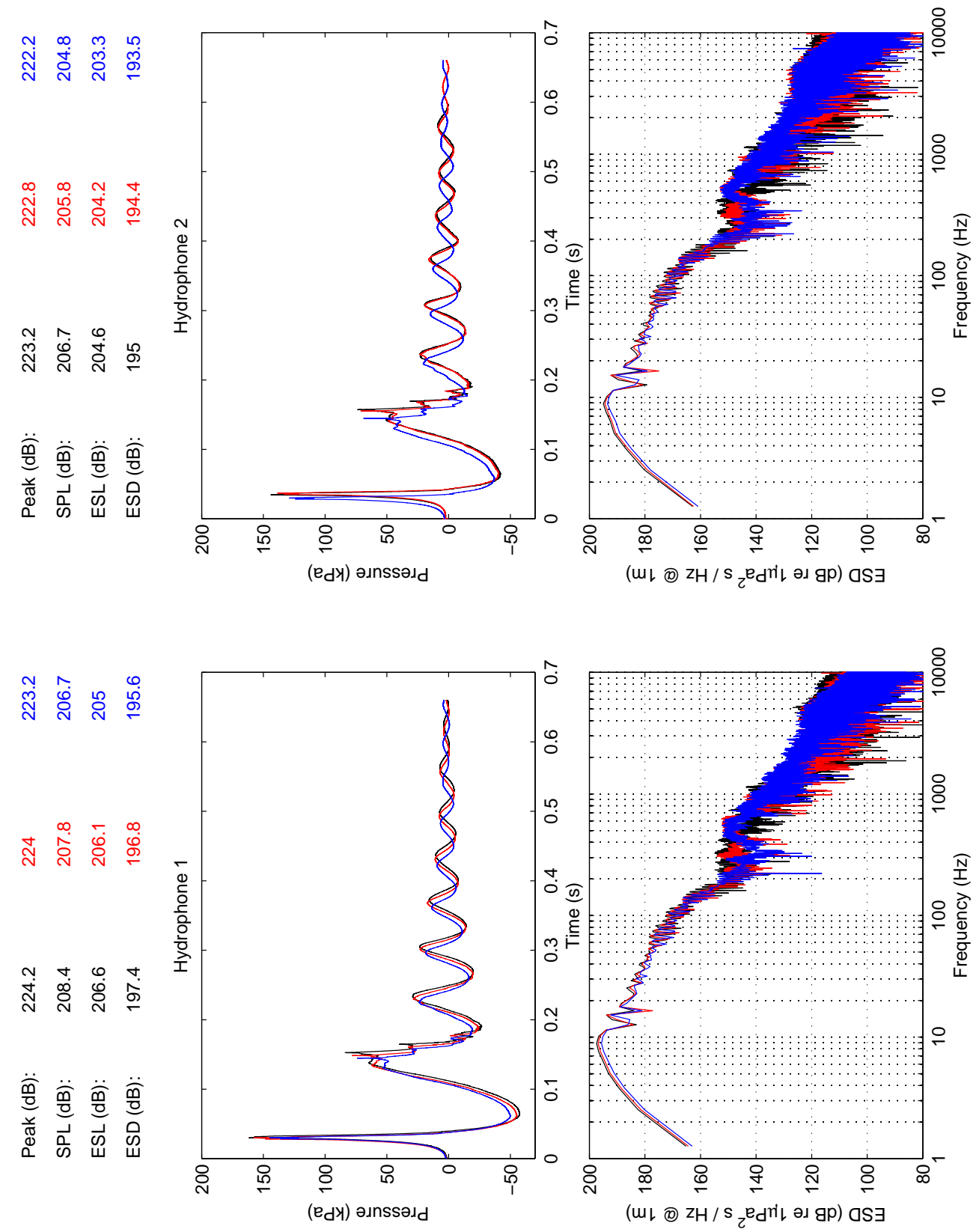

Figure C.19: Time series and spectra of the parameter set described by $240 \mathrm{~L}$ (STP) of the air-hydrogen mixture fired in the 48 " chamber at a depth of $5 \mathrm{~m}$. 


\section{C.4 Oxygen-Hydrogen, 48" Combustion Chamber}

Presented are time series and spectra of each parameter set described by the oxygen-hydrogen mixture fired in the $48^{\prime \prime}$ combustion chamber for various volumes and depths. 

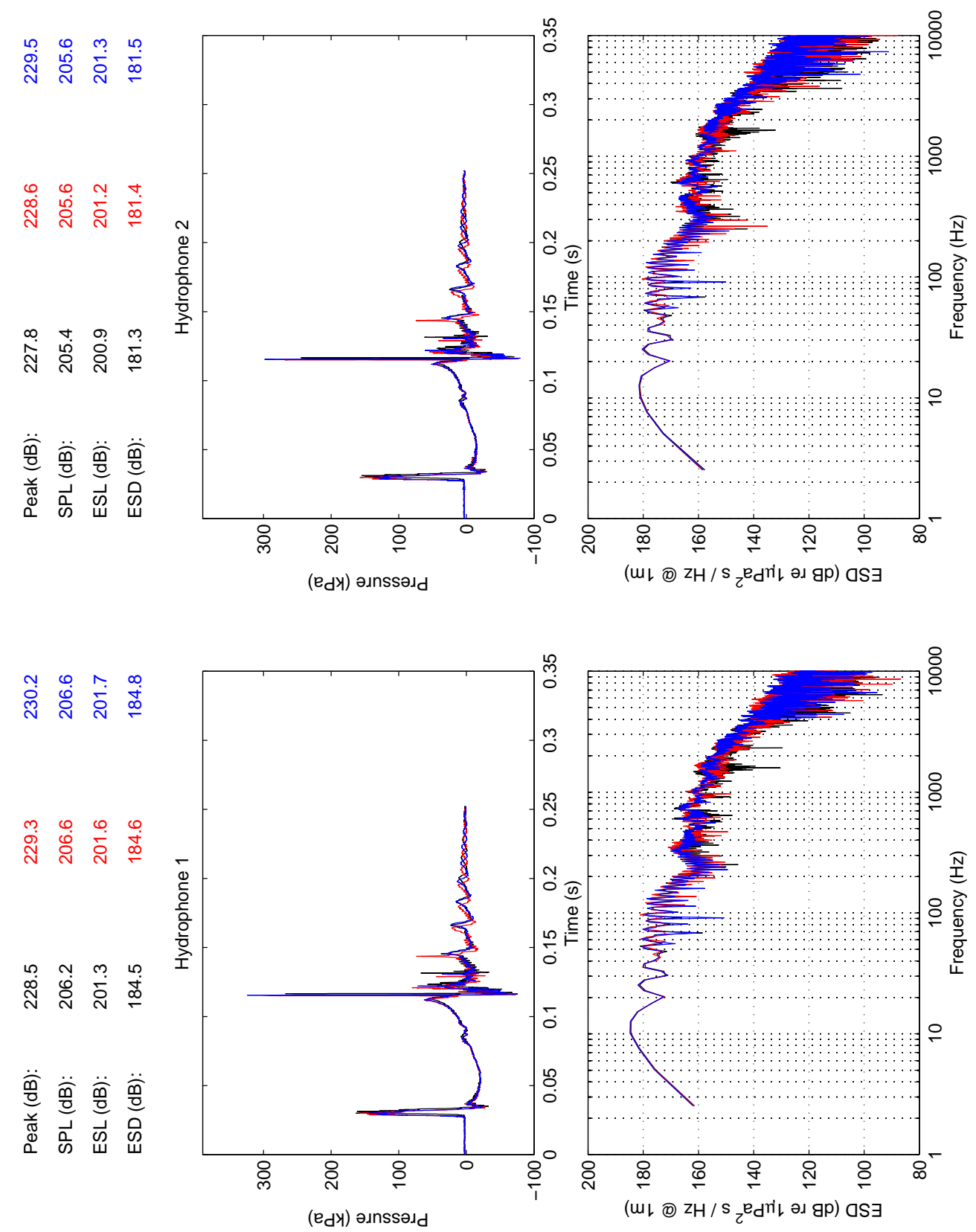

Figure C.20: Time series and spectra of the parameter set described by $60 \mathrm{~L}$ (STP) of the oxygen-hydrogen mixture fired in the $48^{\prime \prime}$ chamber at a depth of $5 \mathrm{~m}$. 

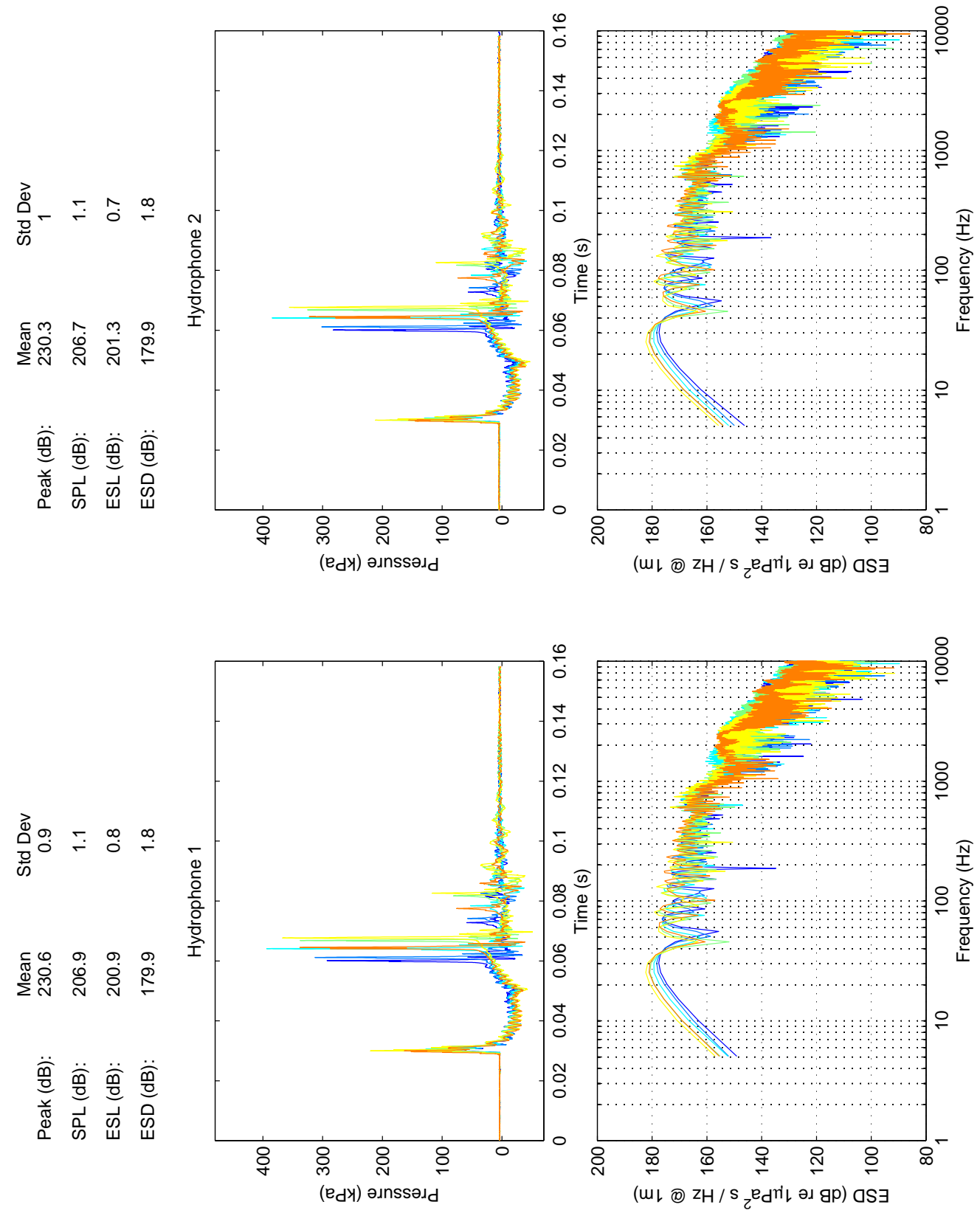

Figure C.21: Time series and spectra of the parameter set described by $60 \mathrm{~L}$ (STP) of the oxygen-hydrogen mixture fired in the 48 " chamber at a depth of $18 \mathrm{~m}$. 

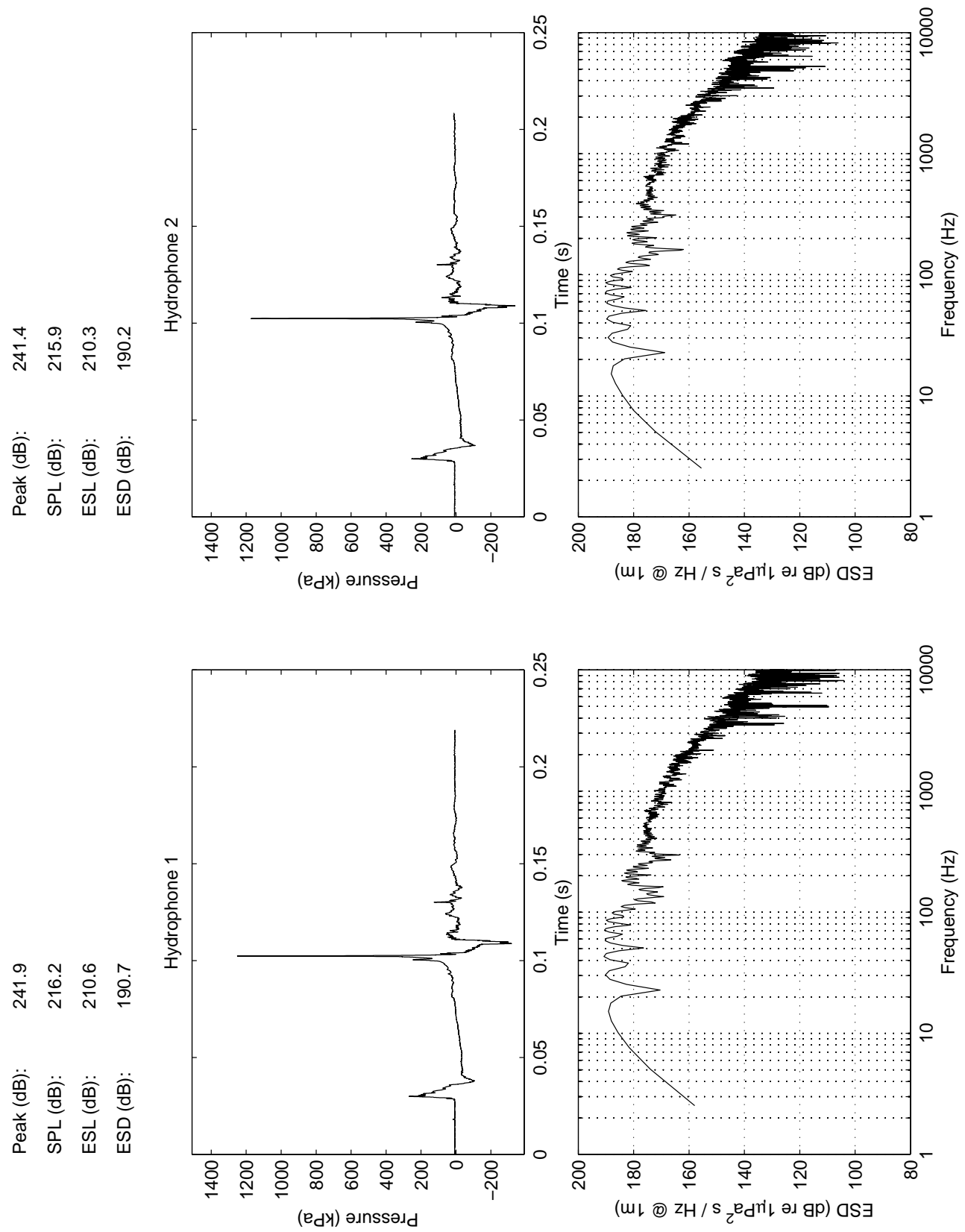

Figure C.22: Time series and spectra of the event described by 150 L (STP) of the oxygenhydrogen mixture fired in the $48^{\prime \prime}$ chamber at a depth of $10 \mathrm{~m}$. 


\section{Appendix D}

\section{ESL Histograms of Deviation from the Mean}

The following histograms show the ESL deviation from the mean of 57 events for various octave bands as described in Section 4.6.3.

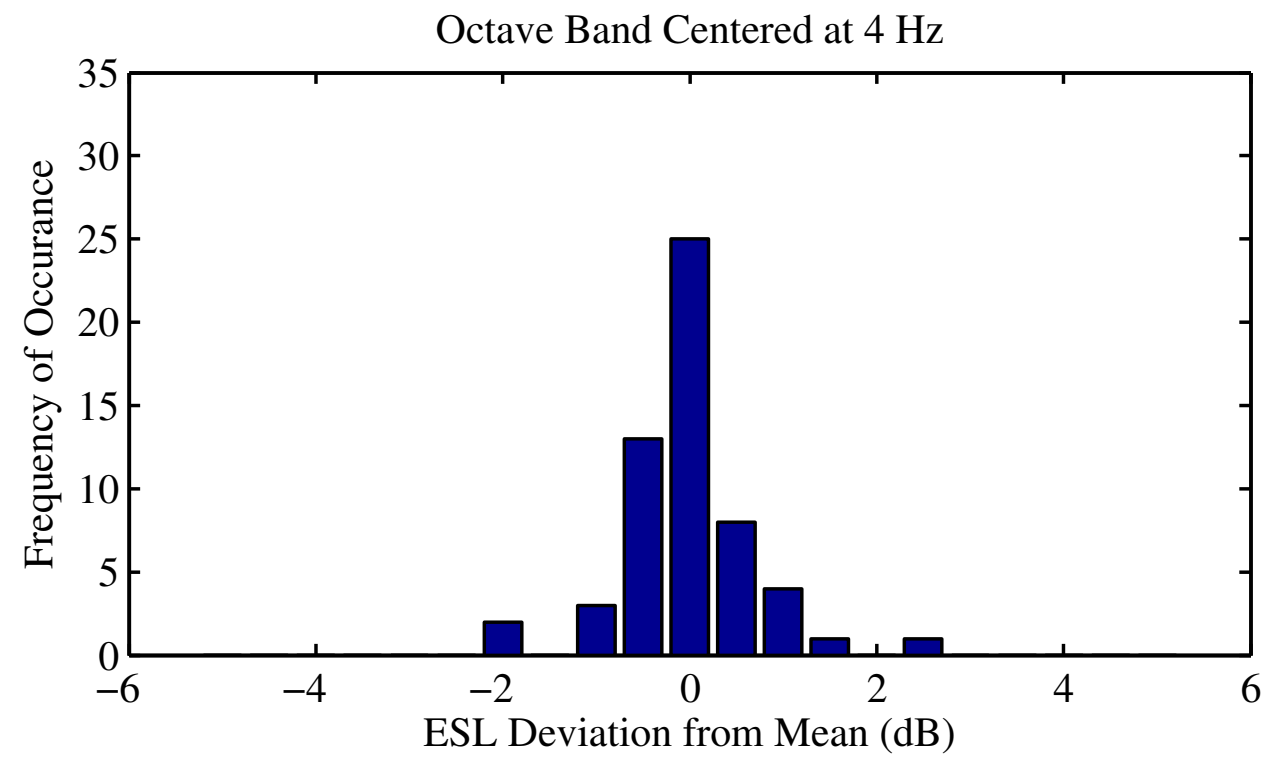

Figure D.1: Histogram of ESL deviation from the mean for the octave band centered at $4 \mathrm{~Hz}$. 


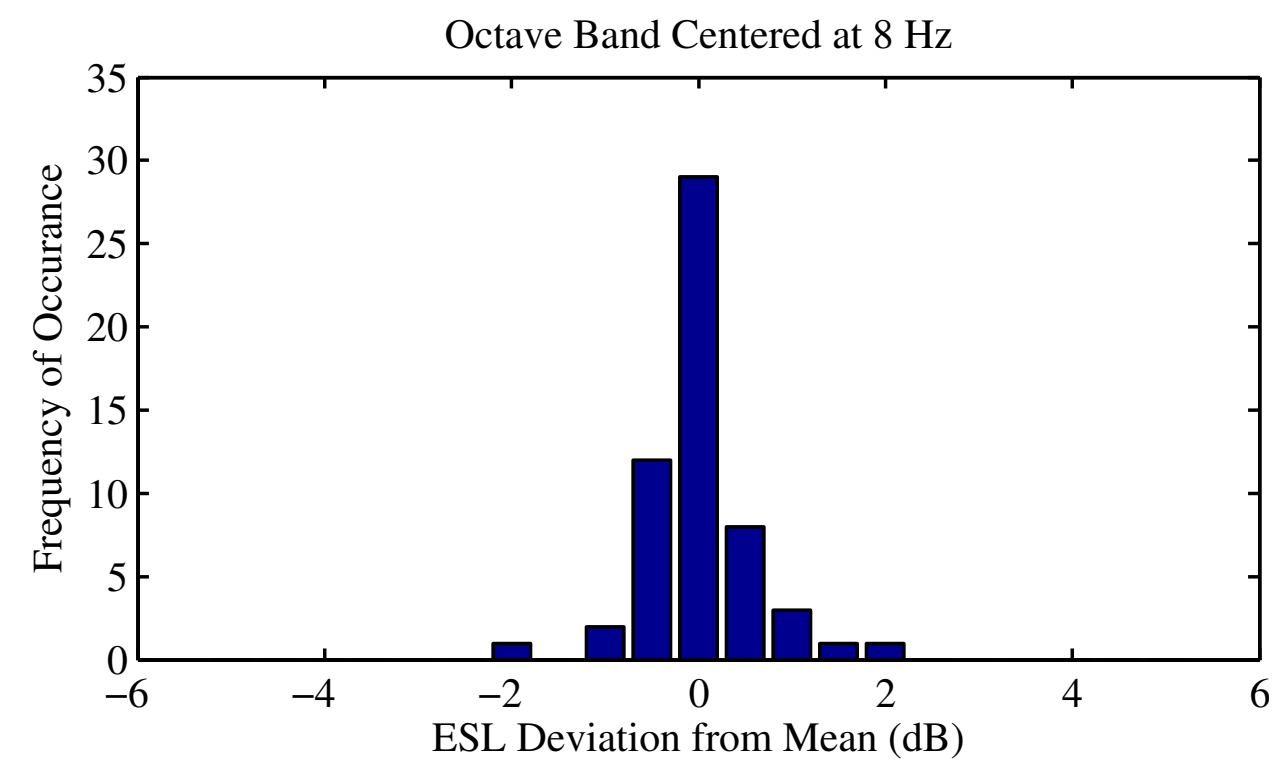

Figure D.2: Histogram of ESL deviation from the mean for the octave band centered at $8 \mathrm{~Hz}$.

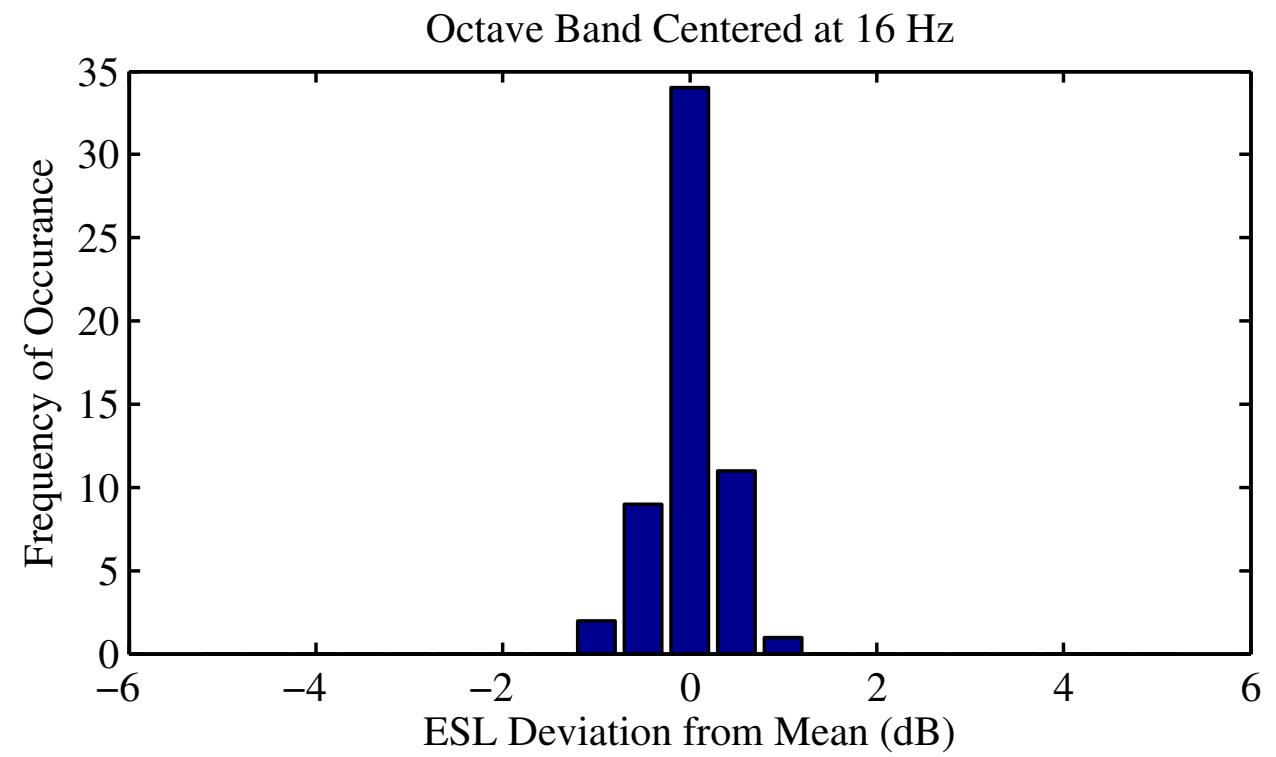

Figure D.3: Histogram of ESL deviation from the mean for the octave band centered at $16 \mathrm{~Hz}$. 


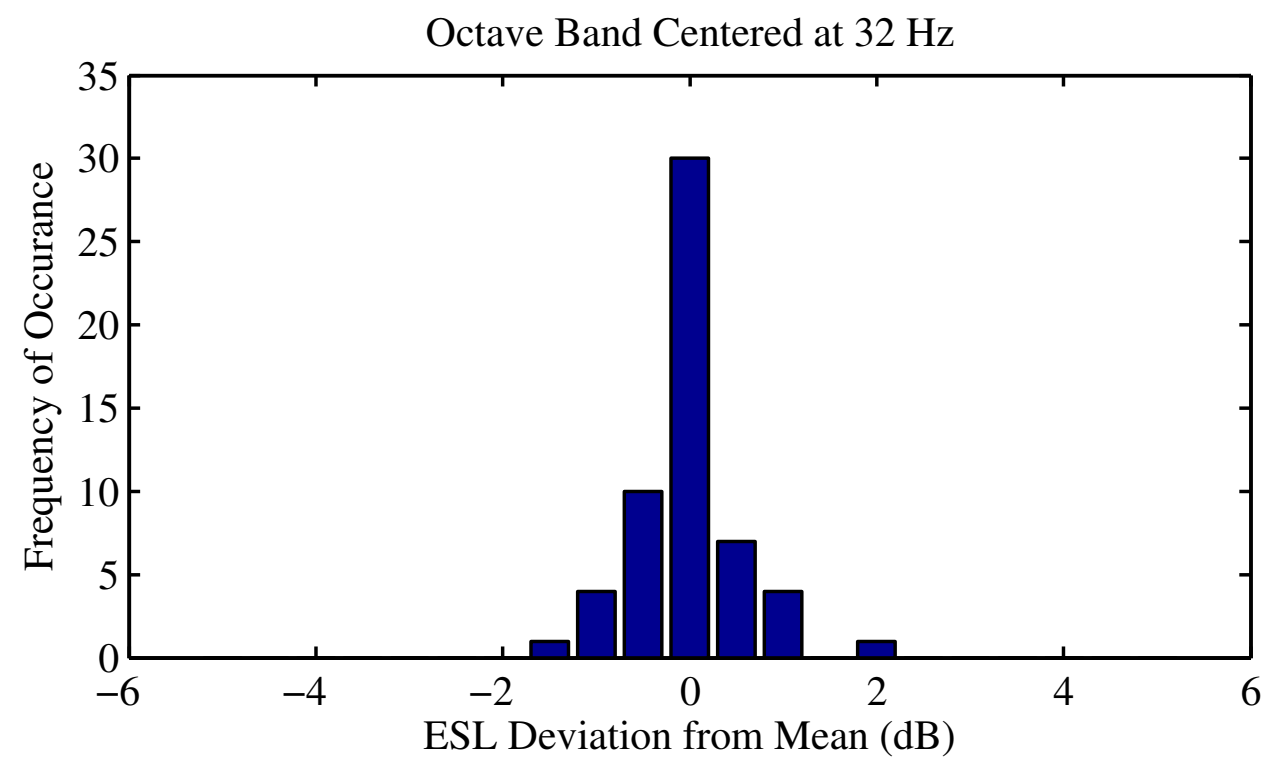

Figure D.4: Histogram of ESL deviation from the mean for the octave band centered at $32 \mathrm{~Hz}$.

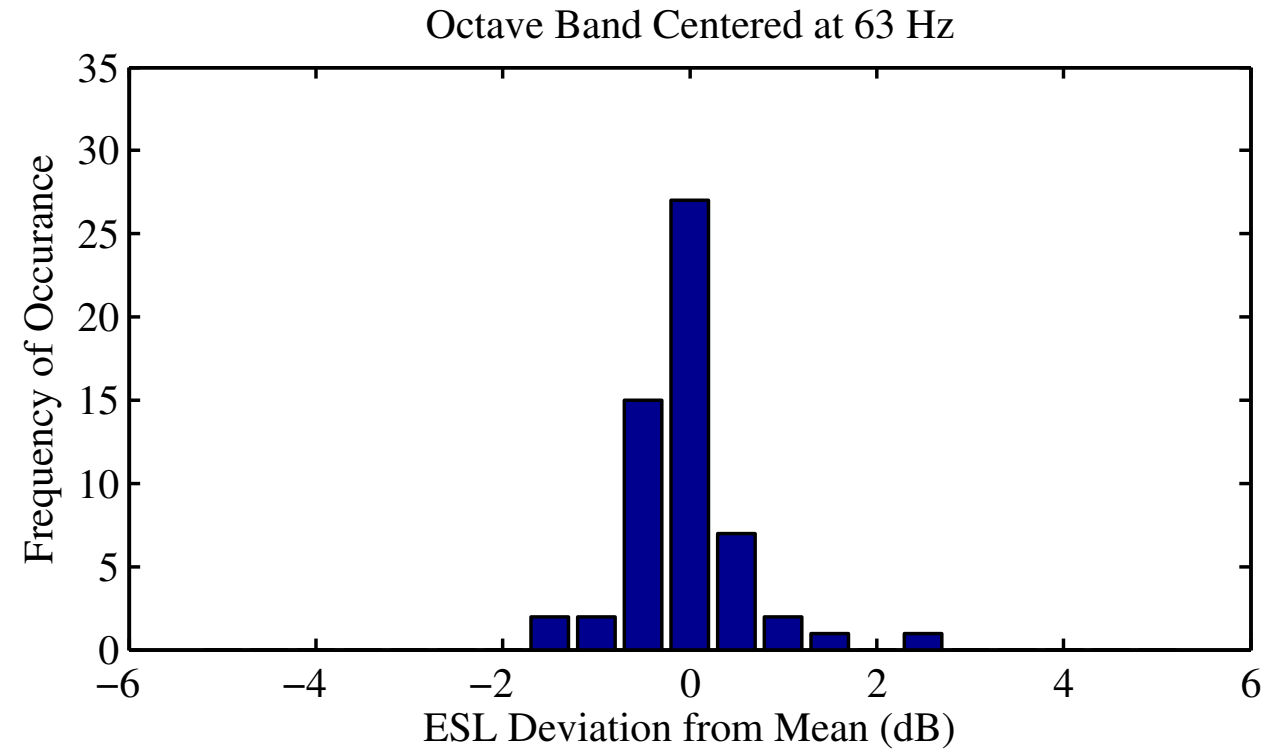

Figure D.5: Histogram of ESL deviation from the mean for the octave band centered at $63 \mathrm{~Hz}$. 


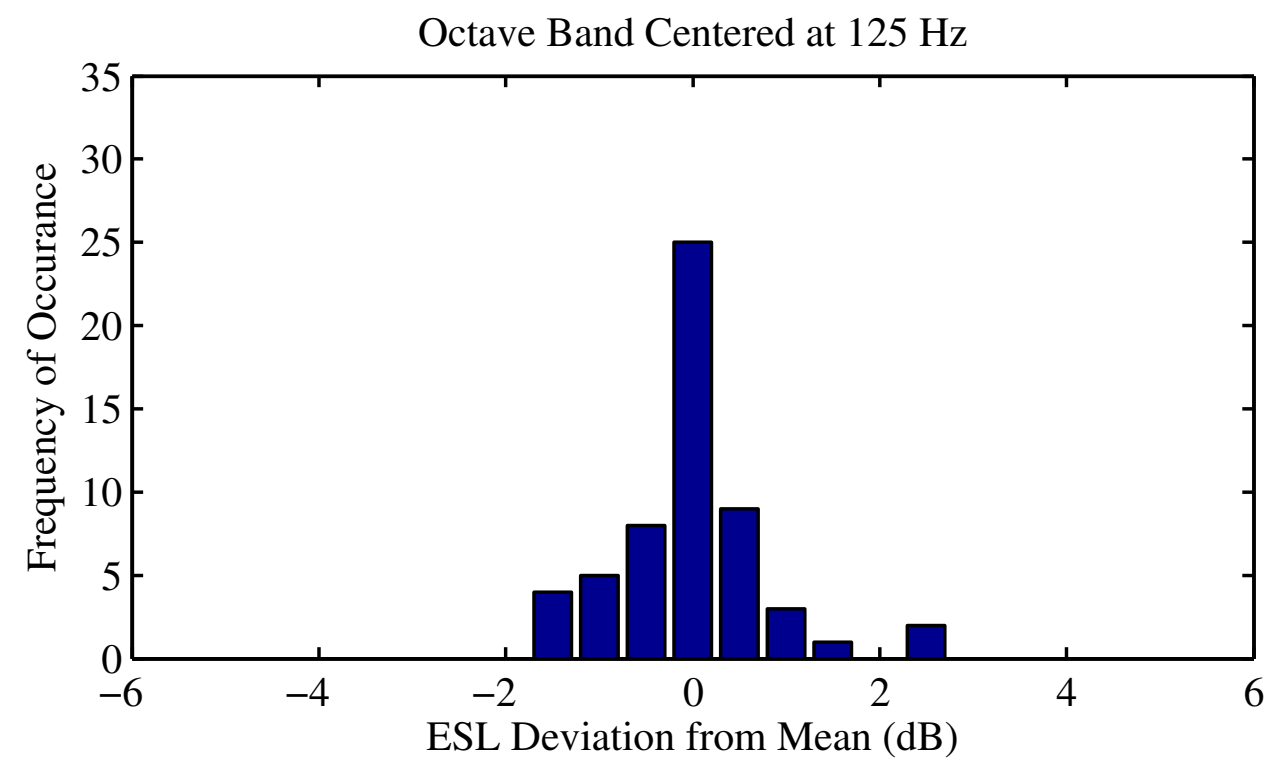

Figure D.6: Histogram of ESL deviation from the mean for the octave band centered at $125 \mathrm{~Hz}$.

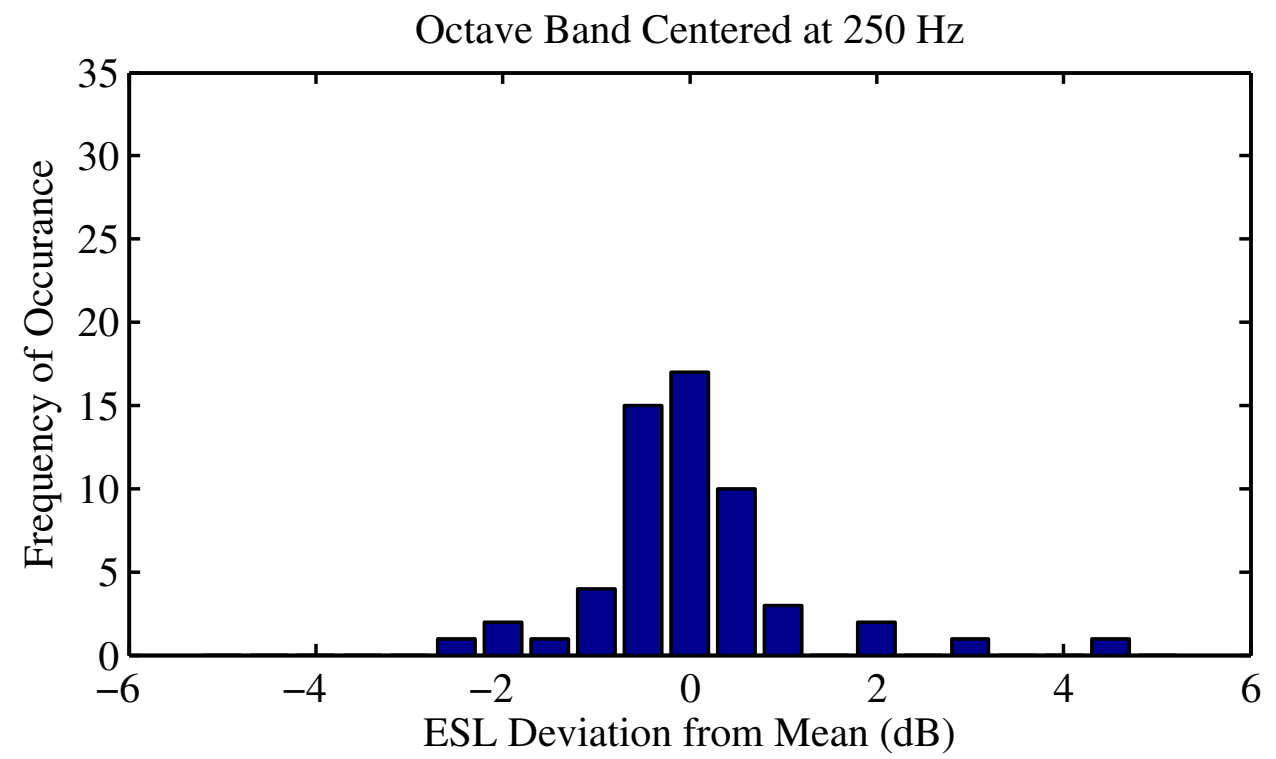

Figure D.7: Histogram of ESL deviation from the mean for the octave band centered at $250 \mathrm{~Hz}$. 


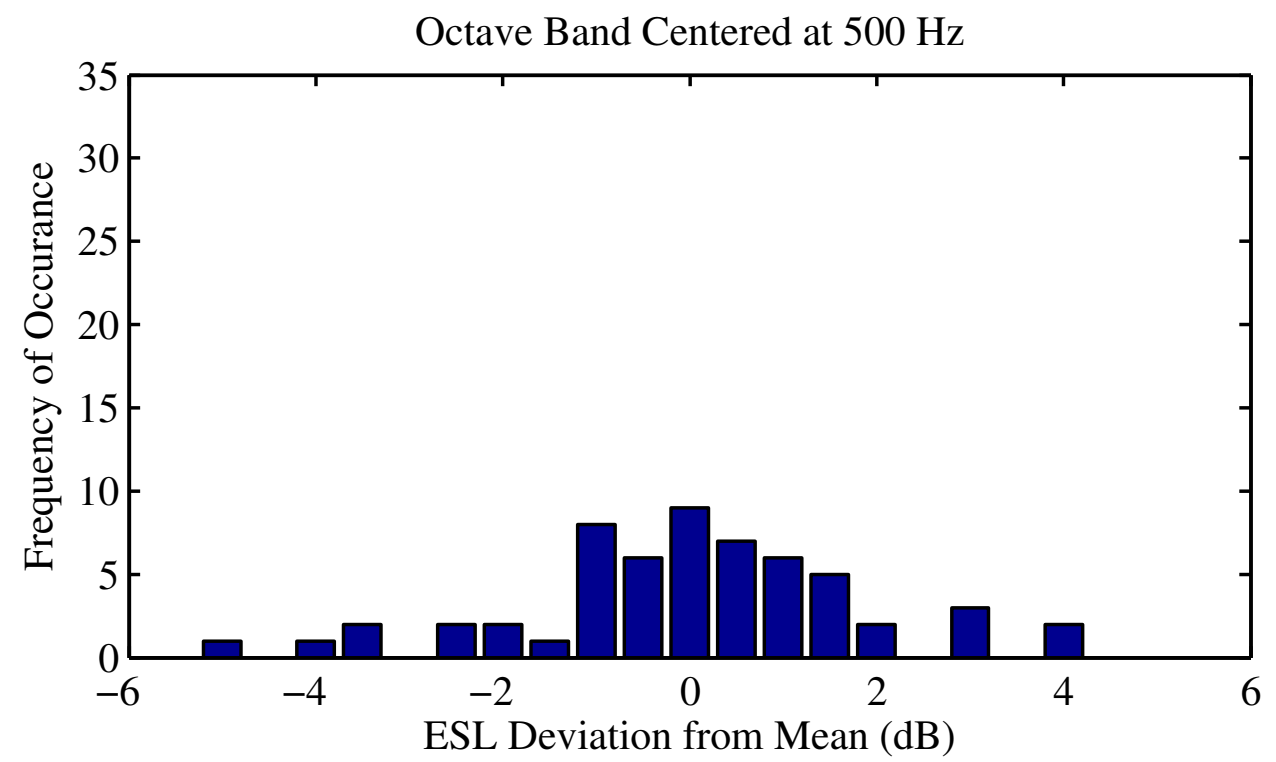

Figure D.8: Histogram of ESL deviation from the mean for the octave band centered at $500 \mathrm{~Hz}$.

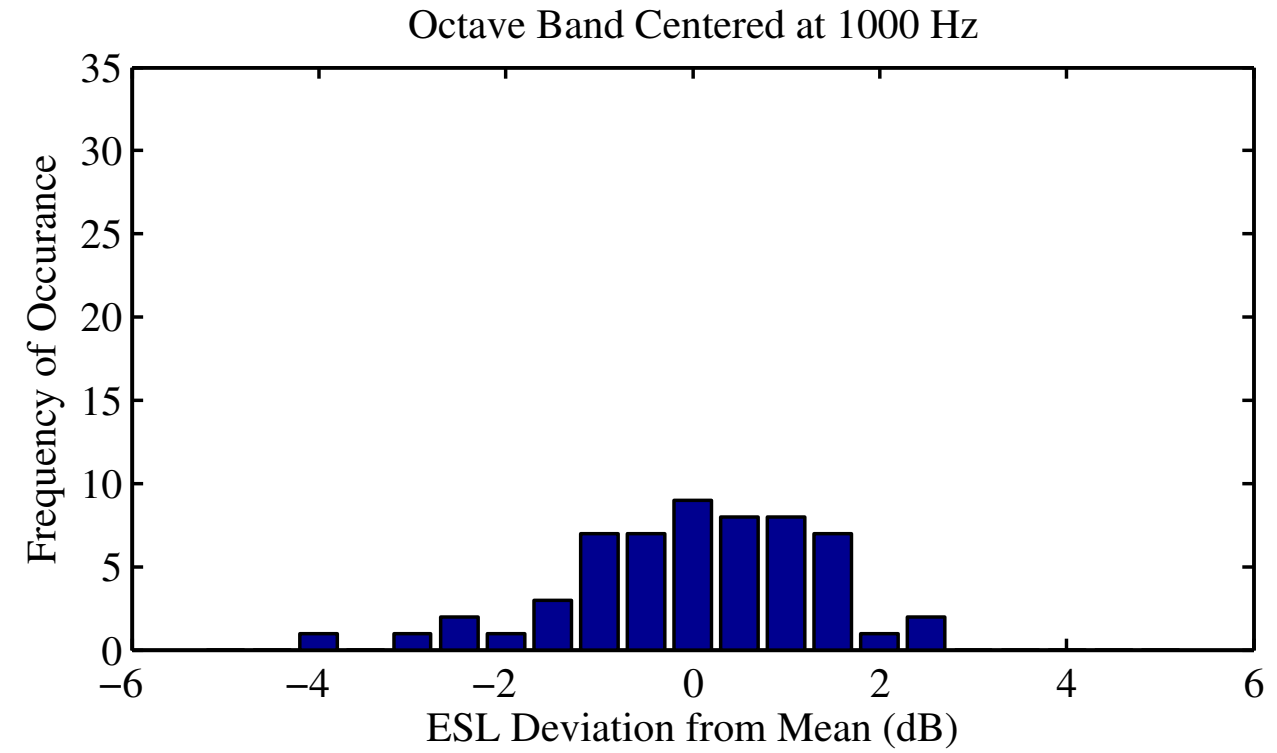

Figure D.9: Histogram of ESL deviation from the mean for the octave band centered at 1000 $\mathrm{Hz}$. 


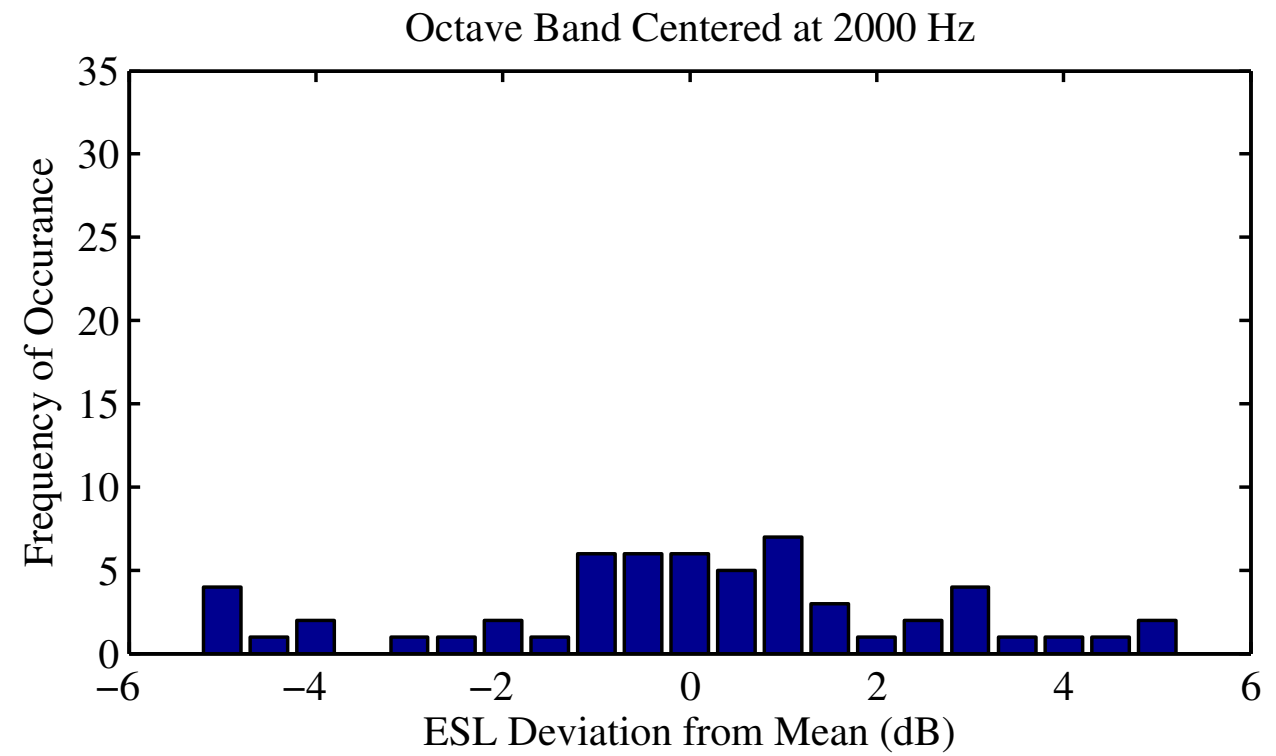

Figure D.10: Histogram of ESL deviation from the mean for the octave band centered at 2000 $\mathrm{Hz}$.

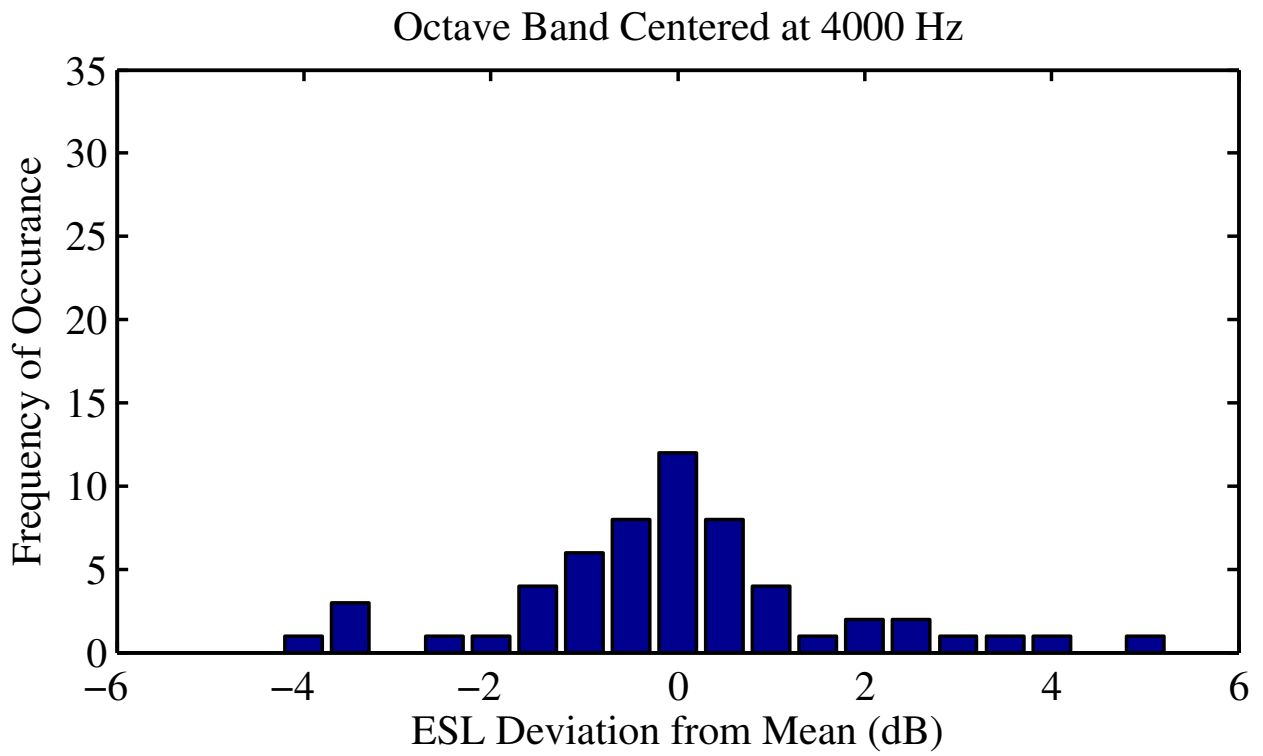

Figure D.11: Histogram of ESL deviation from the mean for the octave band centered at 4000 $\mathrm{Hz}$. 


\section{Bibliography}

[1] E. L. Hamilton, "Geoacoustic modeling of the sea floor", J. Acoust. Soc. Am. 68, 1313-1340 (1980).

[2] J. D. Holmes, W. M. Carey, S. M. Dediu, and W. L. Siegmann, "Nonlinear frequencydependent attenuation in sandy sediments", J. Acoust. Soc. Am. 121, EL218-EL222 (2007).

[3] D. P. Knobles, R. A. Koch, J. H. Miller, and G. R. Potty, "Evidence for nonlinear frequency dependence of attenuation in an east china sea environment", J. Acoust. Soc. Am. 115, 2551-2551 (2004).

[4] N. R. Chapman, "Source levels of shallow explosive charges", J. Acoust. Soc. Am. 84, 697702 (1988).

[5] D. M. Donskoy and J. E. Blue, "A new concept of a low-frequency underwater sound source", J. Acoust. Soc. Am. 95, 1977-1982 (1994).

[6] A. K. Morozov and D. C. Webb, "A sound projector for acoustic tomography and global ocean monitoring", IEEE Journal of Oceanic Engineering 28, 174-185 (2003).

[7] M. B. Dobrin, Introduction to Geophysical Prospecting, 4th. edition (McGraw-Hill) (1988).

[8] J. H. Hammond Jr., Submarine Sound Transmitter, U.S. Patent 1500243 (1924).

[9] E. P. Anderson, Electrolytic-Ignition Underwater Sound Source, U.S. Patent 3099813 (1963).

[10] T. E. Owen, Directional Underwater Acoustic Pulse Source, U.S. Patent 5229977 (1993).

[11] P. S. Wilson, The Combustive Sound Source, Master's thesis, The University of Texas at Austin (1994).

[12] P. Wilson, J. Ellzey, and T. Muir, "Experimental investigation of the combustive sound source", IEEE Journal of Oceanic Engineering 20, 311 -320 (1995).

[13] M. A. Liberman, Introduction to Physics and Chemistry of Combustion: Explosion, Flame, Detonation (Springer) (2008).

[14] I. Glassman and R. Yetter, Combustion, 4th edition (Academic Press) (2008).

[15] P. B. West, Regenerative Combustion Device, U.S. Patent 6705425 (2004).

[16] P. S. Wilson, T. G. Muir, J. A. Behrens, and J. L. Elizey, "Applications of the combustive sound source", J. Acoust. Soc. Am. 97, 3298(A) (1995). 
[17] Naval Air Systems Command, Description, Operation and Handling Instructions: Signals, Underwater Sound (SUS), second edition (1985).

[18] D. Horton and V. Ho, 2 Killed In Explosion on Ship - Blast Off Grays Harbor, The Seattle Times (1992).

[19] J. J. Wakeley, "Coherent ray tracing - measured and predicted shallow-water frequency spectrum", J. Acoust. Soc. Am. 63, 1820-1823 (1978).

[20] L. Rayleigh, "On the pressure developed in a liquid during the collapse of a spherical cavity", Phil. Mag. 34, 94-98 (1917).

[21] H. F. Willis, "Underwater explosions: Time interval between successive explosions", Br. Admir. Rep. WA-47-21 (1941). 


\section{VITA}

Andrew Reed McNeese was born in Little Rock, Arkansas. After completing his work at Central Arkansas Christian High School, Little Rock, Arkansas, in 2003, he entered Abilene Christian University in Abilene Texas. In 2005 he transferred to the University of Texas at Austin. He received the degree of Bachelor of Science from the University of Texas in May, 2008. In September, 2008, he entered the Graduate School at the University of Texas at Austin.

Permanent Address: 41 Kings River

North Little Rock, AR 72116

This thesis was typed by the author. 